ESAIM: PROCEEDINGS AND SURVEYS, April 2020, Vol. 67, p. 285-335

Vincent Calvez, Céline Grandmont, Eva Locherbach, Clair Poignard, Magali Ribot, Nicolas Vauchelet Editors

\title{
OPINION PROPAGATION ON SOCIAL NETWORKS: A MATHEMATICAL STANDPOINT*
}

\author{
H. LAVEnANT ${ }^{1}$ AND B. MAURY ${ }^{2}$
}

\begin{abstract}
These lecture notes address mathematical issues related to the modeling of opinion propagation on networks of the social type. Starting from the behavior of the simplest discrete linear model, we develop various standpoints and describe some extensions: stochastic interpretation, monitoring of a network, time continuous evolution problem, charismatic networks, links with discretized Partial Differential Equations, nonlinear models, inertial version and stability issues. These developments rely on basic mathematical tools, which makes them accessible at an undergraduate level. In a last section, we propose a new model of opinion propagation, where the opinion of an agent is described by a Gaussian density, and the (discrete) evolution equation is based on barycenters with respect to the Fisher metric.

Résumé. Ce support de cours traite de questions mathématiques en lien avec la modélisation de la propagation d'opinion dans des réseaux sociaux. À partir du modèle le plus simple, discret et linéaire, nous développons des points de vue divers et proposons des extensions: interprétation stochastique, contrôle des opinions sur un réseau, modèle d'évolution continu en temps, réseaux «charismatiques », liens avec les équations aux dérivées partielles discrétisées, modèles non linéaires, modèle avec inertie et questions de stabilité. Ces développements reposent sur un bagage limité d'outils mathématiques, de telle sorte que l'essentiel est accessible au niveau licence. Dans une dernière section, nous proposons un nouveau modèle de propagation d'opinion, où l'opinion est décrite par une densité gaussienne, et l'évolution discrète est basée sur la notion de barycentre selon la métrique de Fisher.
\end{abstract}

\section{INTRODUCTION}

We aim at modeling propagation of opinion over networks of the social type. As a typical example, consider a collection of voters before the second round of a runoff voting process. The opinion of each voter, i.e. their propensity to vote for one of the two candidates, can be encoded by a number in some interval, the ends of which correspond to firm intentions. Other sorts of opinion can be encoded by a number, like the expected temperature rise in 20 years due to global warming. In both cases, each agent is likely to progressively change their opinion by interacting with other people. In this context, modeling the changes and the propagations of opinion consists in identifying the agents who influence a given individual, and setting how the opinion of this very individual are influenced by the opinions of those influential neighbors. The evolution models which are obtained in this way present some similarities with diffusion processes over networks, and the equation which

\footnotetext{
* These notes follow a series of lectures which were given at two summerschools: Cemracs 2018, in Marseille, and Mathematical Summer in Paris, july 2018, at École Normale Supérieure de Paris, PSL University.

${ }^{1}$ Department of Mathematics, University of British Columbia, Vancouver BC Canada

2 DMA, École normale supérieure, CNRS, PSL University, 75005 Paris, France

\& Laboratoire de Mathématiques d'Orsay, Univ. Paris Sud, CNRS, Univ. Paris-Saclay, Bâtiment 307, 91405 Orsay Cedex
}

(C) EDP Sciences, SMAI 2020

This is an Open Access article distributed under the terms of the Creative Commons Attribution License (http://creativecommons.org/licenses/by/4.0), which permits unrestricted use, distribution, and reproduction in any medium, provided the original work is properly cited. 
will play a central role in these notes can be seen considered as a heat equation canonically associated to the network. Yet, a crucial characteristic of social networks is the absence of symmetry, which makes them quite different from standard diffusion processes. The influence of an agent $x$ over an agent $y$ is not correlated to the influence that $y$ exerts on $x$. This relation might even be fully asymmetric, i.e. $x$ influences $y$ whereas $x$ does not account for $y$ (like in the Follower relation in Twitter network).

Let us emphasize that our work is rather academic. We aim here at building a theoretical framework rather than confronting the models with experimental data. The topic of propagation of information over a network has been widely studied, both from the modeling and statistical standpoints, and we refer to Section 10 at the end of this article for a brief bibliographical survey. Compared to a large part of the existing literature, we will concentrate on (over)simplified models, based on assumptions which are questionable, and we will not try to justify these assumptions except by relying on the reader's common sense. On the other hand, since the objects studied in these notes have a strong mathematical structure, we will be able to draw a lot of conclusions without relying on any numerical computations. Moreover, we hope that the structure of the models will be apparent enough so that the reader would know how additional features could be incorporated.

More specifically, we will study evolution of numerical quantities (the opinion is encoded as a real number) which will be defined on the vertices of weighted directed graphs. Each vertex will correspond to an entity (individual, group of individuals, animal, ...) and we shall consider that there is an arrow (or directed edge) from $x$ to $y$ whenever $x$ is influenced by $y$. The asymmetry of the propagation of information is directly reflected by the directed character of the graph: $x \rightarrow y$ does not imply $y \rightarrow x$. We will mainly concentrate on linear evolution models: in fact, our work is closely related to matrix theory and Markov chains, though the language and questionings will differ. Through these notes, we will revisit some well known results, but we hope that the reader will appreciate the relevance of the application of these results to our problem. Some proofs may appear over-complicated at first glance: indeed, we have tried to choose the ones which rely on relevant modeling ideas, and which are robust enough to be extended to more complicated settings, rather than "one-line" proofs which would be too specific and rely on a general theory. As an example, we have tried to avoid as much as possible to talk about reduction of linear operators, at least in the first sections.

We advise the reader to first take a look at Section 1 it provides the vocabulary about weighted directed graphs, and present the simplest linear model. All the remaining sections are basically variations about the model described in Section 1, and can be read independently from one another (up to some cross-references which are mainly contained in remarks). In order to facilitate the reading, we have gathered most bibliographical notes in a dedicated section (Section 10 ) at the end of these lecture notes.

\section{Propagation of information over a network}

We consider here a simple model for influence spreading over a network, the nodes of which are entities characterized by a real number which measures an opinion with respect to some subject. This number might for exemple represent the odds that a national team is likely to win some competition, or individual tendency to vote for some candidate at an election. It may also correspond to some specific quantity, the value of which is involved in a public debate, like the expected temperature rise in 20 years due to global warming. The model is quite crude from two standpoints: the evolution problem is linear and the opinion is represented by a single real number. We refer to Section 7 for nonlinear evolution models, and to Section 9 for a model based on the representation of the opinion by a probability measure, allowing to account for the degree of belief.

We consider a set $V$ of $N$ individuals, we denote by $u_{x}^{t}$ the opinion of the individua $\left.\right|^{1} x$ at some time $t$, and by $u^{t}=\left(u_{x}^{t}\right)_{x \in V}$ the collection of opinions over $V$. We first consider a discrete evolution setting: $t$ corresponds to successive times $0,1, \ldots, k, \ldots$ The influence of some $y \in V$ upon individual $x$ is quantified by $K_{x y} \in[0,1]$,

\footnotetext{
${ }^{1}$ We shall use interchangeably the words "person", "individual", "agent" or even "vertex".
} 
and we assume that these coefficients sum up to 1 :

$$
\sum_{y \in V} K_{x y}=1 \quad \forall x \in V
$$

The collection $\left(K_{x y}\right)_{(x, y) \in V^{2}}$ of nonnegative coefficients with the normalization above will be called, following the standard terminology, a stochastic matrix (or row stochastic matrix).

A directed graph $(V, E)$ can be associated to the field of influences $\left(K_{x y}\right)$, where the set of directed edges $E \subset V \times V$ is defined by

$$
E=\operatorname{supp}\left(K_{x y}\right)=\left\{(x, y) \in V \times V, K_{x y}>0\right\} .
$$

We shall write $x \rightarrow y$ if $K_{x y}>0$, which means that $x$ listens to $y$, or $x$ follows $y$, or more generally $x$ is influenced by $y$. With this convention, the information or influence flows upstream arrows.

We shall use extensively the notions of path and cycle:

Definition 1.1. (Path / cycle / loop)

A path is a finite sequence of connected vertices

$$
x_{0}, x_{1}, \ldots, x_{n} \text { with }\left(x_{i}, x_{i+1}\right) \in E \quad \forall i=0, \ldots, n-1, n \geq 1 .
$$

When there exists a path from $x$ to $y$, we shall write $x-\cdot \rightarrow y$. Similarly, if there is a path from $x$ to any vertex of a subset $X \subset V$, we shall write $x-\cdot \rightarrow X$, and $V-\cdot \rightarrow X$ if any vertex is connected to $X$.

A cycle is a path which ends where it starts, and which contains at least one vertex distinct from the origin.

A loop is a single edge of the form $(x, x)$.

A strongly connected graph is such that there exists a path from any $x$ to any $y$, i.e. $V-\cdot \rightarrow V$.

Note that $K_{x x}$ may be positive (i.e. there might be loops in the network), which represents some sort of inertia, or resistance of $x$ to external influence, possibly up to full disregard of opinion displayed by others. We shall denote by $\Gamma \subset V$ the subset of all vertices which only point to themselves:

$$
\Gamma=\left\{x \in V, K_{x x}=1\right\},
$$

and by $\stackrel{\circ}{V}=V \backslash \Gamma$ the set of interior vertices. In terms of influence, $\Gamma$ corresponds to individuals who do not listen to anybody but themselves. We shall call those agents influencer ${ }^{2}$.

\section{Evolution model in the discrete setting}

Given an initial collection of opinions $\left(u_{x}^{0}\right)_{x \in V}$, we consider that the collection of opinions progressively evolves according to

$$
u_{x}^{k+1}=\sum_{x \rightarrow y} K_{x y} u_{y}^{k} \quad \forall x \in V .
$$

Note that the ' $x \rightarrow y$ ' under the sum sign is not strictly mandatory since $K_{x y}=0$ as soon as $(x, y) \notin E$. We may sometimes drop it, and simply write $\sum K_{x y} u_{y}^{k}$.

\footnotetext{
${ }^{2}$ Those agents may also be qualified as stubborn, like in 33, since they never change their mind. We shall nevertheless keep the term influencer to account for the fact that they may be seen as actors who exert a persistent influence over the network, while being possibly controlled by an external planner (see Section 3 .
} 
Definition 1.2. (Fixed point)

We say that $u=\left(u_{x}\right)_{V} \in \mathbb{R}^{V}$ is a fixed point for (2) whenever

$$
u_{x}=\sum_{x \rightarrow y} K_{x y} u_{y} \quad \forall x \in V
$$

The set of fixed points is a linear space, the dimension of which can take any value between 1 and the number $N$ of vertices, depending on the structure of $\left(K_{x y}\right)$. Any such fixed point will be said to be harmonic with respect to $K=\left(K_{x y}\right)$.

A fixed point is a configuration of opinions where no evolution takes place, where an equilibrium is reached. Any vector of the form $e=(1, \ldots, 1)$ is a fixed point, and so is $\lambda e$ for any $\lambda \in \mathbb{R}$. Such a fixed point is called a consensus for obvious reasons: all agents have the same opinion. There might be other fixed points. For instance, in the situation where the graph contains only loops $(\Gamma=V)$, any vector is a fixed point. In terms of opinion, it simply means that, when there is no communication at all between the members of a group, all individuals keep their initial opinion, which is arbitrary.

Remark 1.3. Equation (2) can be written

$$
u_{x}^{k+1}=u_{x}^{k}+\sum_{x \rightarrow y} K_{x y}\left(u_{y}^{k}-u_{x}^{k}\right)=u_{x}^{k}+\sum_{x \rightarrow y} F_{y x}^{k}
$$

where $F_{y x}^{k}=K_{x y}\left(u_{y}^{k}-u_{x}^{k}\right)$ can be interpreted as a flux of opinion from $y$ to $x$. The definition of this flux can be seen as some sort of Ohm's Law (or Poiseuille's Law in a fluid dynamics context) which states a linear relation between a flux and a difference of potentials (or difference of pressures in the context of fluid dynamics). From this standpoint, $K_{x y}$ appears as a conductance parameter which characterizes the connection $x \rightarrow y$ in terms of opinion propagation from $y$ to $x$. Note that, unlike Ohm's and Poiseuille's laws, the present one does not rely on symmetric interactions. Actually in many asymmetric social networks, it commonly happens that $K_{x y}>0$ while $K_{y x}=0$ (fully asymmetric influence relation).

Proposition 1.4. (Maximum principle, dynamic version)

Let an initial state $u^{0} \in \mathbb{R}^{V}$ be given, and let $u^{1}, \ldots, u^{k}, \ldots$ be the fields recursively defined according to (2). Then, for any $k \geq 0$, for any $x \in V$,

$$
u_{x}^{k+1} \in\left[\min u^{k}, \max u^{k}\right] \subset\left[\min u^{0}, \max u^{0}\right] .
$$

Proof. Since, for any $x$, the $K_{x y}$ 's are nonnegative and sum up to 1, Equation (2) express that $u_{x}^{k+1}$, is a convex combination of the $u_{y}^{k}$ 's. As a direct consequence, one has

$$
u_{x}^{k+1} \in\left[\min u^{k}, \max u^{k}\right] .
$$

The property follows by induction on $k$.

The maximum principle can also be expressed in a static form, see Proposition 1.9 below.

Remark 1.5. The opinion variable $u$ is not conservative, in the sense that the sum

$$
\sum_{x \in V} u_{x}^{k}
$$

which is the mean opinion (up to a factor $1 / N$ ), varies with $k$ in general. Let us give an example of a highly non-conservative evolution: consider a network of $N$ agents, such that the set $\Gamma$ of influencers reduces to a 
single node $x$. Suppose also that all nodes are connected to $x$, i.e. for any $y \in V$, there exists a path (see Definition 1.1) from $y$ to $x$. Assume that the initial opinion of $x$ is 1 , whereas the opinion of all others is 0 . If $N$ is large, the initial mean opinion is close to 0 , while the evolution process $(2)$ shall make this mean opinion converge to 1 , as a consequence of Proposition 1.10 proved below.

Remark 1.6. (Thermodynamics of opinion propagation)

If one sees the sum of opinion as an energy, the previous remark shows that the model does not obey the First Law of Thermodynamics: the total energy is not conserved. On the other hand, the Ohm-like law (see Remark 1.3 expresses that the opinion flows from higher values to smaller values, thereby tending to make the distribution more uniform by decreasing the maximum and increasing the minimum (Maximum Principle in Proposition 1.4). From this standpoint, the model can be said to obey the Second Law of Thermodynamics, although a proper entropy may not always be defined, and convergence toward an equilibrium state may be ruled out. As we shall see in Section 2, the transpose problem will present a reverse structure (see in particular Diagram (8)).

Because of its hyper-academic character, we shall not consider in this section the non-diffusive case, i.e. the case of graphs which correspond to a mapping, with $K_{x y}$ taking values in $\{0,1\}$. Yet, for the sake of mathematical completeness, we develop this situation in Appendix A.

Let us first consider the situation of a complete graph, i.e. when everyone listens to everybody.

Proposition 1.7. (Convergence to a consensus)

$W e$ assume that $K_{x y}>0$ for all $(x, y) \in V \times V$. In other words, $E=V \times V$. In that case the solution to (2) converges to a uniform field.

Proof. It holds that

$$
u_{x}^{k+1}=\sum_{y} K_{x y} u_{y}^{k}=\sum_{y} K_{x y} \max u^{k}+\sum_{y} K_{x y} \underbrace{\left(u_{y}^{k}-\max u^{k}\right)}_{\leq 0} \leq \max u^{k}+K_{x y_{m}}\left(u_{y_{m}}^{k}-\max u^{k}\right),
$$

where $y_{m}$ realizes the minimum of $u_{y}^{k}$. As a consequence

$$
u_{x}^{k+1} \leq \max u^{k}+K_{\min }\left(\min u^{k}-\max u^{k}\right),
$$

where $K_{\min }>0$ is the smallest of the coefficients $K_{x y}$. Thus

$$
\max u^{k+1} \leq \max u^{k}+K_{\min }\left(\min u^{k}-\max u^{k}\right),
$$

Similarly

$$
\begin{gathered}
u_{x}^{k+1}=\sum_{y} K_{x y} u_{y}^{k}=\sum_{y} K_{x y} \min u^{k}+\sum_{y} K_{x y}\left(u_{y}^{k}-\min u^{k}\right) \\
\geq \min u^{k}+K_{\min }\left(\max u_{k}-\min u_{k}\right)
\end{gathered}
$$

so that

$$
\min u^{k+1} \geq \min u^{k}+K_{\min }\left(\max u^{k}-\min u^{k}\right) .
$$

Taking the difference between the two inequalities, we obtain

$$
0 \leq \max u^{k+1}-\min u^{k+1} \leq\left(1-2 K_{\min }\right)\left(\max u^{k}-\min u^{k}\right) .
$$

Since $1-2 K_{\min } \in[0,1)$ (as soon as $N \geq 2$, as the graph is complete, it holds that $K_{\min } \leq 1 / 2$ ), it proves that the sequences $\left(\max u^{k}\right)$ and $\left(\min u^{k}\right)$ are adjacent. Now as $\max u^{k}$ is non-increasing, and $\min u^{k}$ is non-decreasing, both converge to the same value, which implies convergence of the sequence $\left(u^{k}\right)$ toward some $u \in \mathbb{R}^{V}$, which is uniform (the minimum and the maximal values are the same). 
The condition on the coefficients $K_{x y}$ can be alleviated, as stated by the next corollary.

Corollary 1.8. Assume that there exists an integer $m$ such that, for any vertices $x$ and $y$, there exists a path of length exactly $m$ which connects $x$ to $y$. Then, for any initial condition, the solution to (2) converges to a uniform field.

Proof. By a slight abuse of notations, we denote by $K$ the mapping which defines the relation (2), and by $K^{m}$ the mapping $K \circ K \circ \cdots \circ K$ ( $m$ times). The previous proposition can be applied to this new process, which proves convergence of $\left(K^{m}\right)^{k} u_{0}$ to a uniform field. Since $K$ is continuous and preserves uniform fields, we have that $K^{m k+1} u^{0}=K\left(K^{m k} u^{0}\right)$ converges to the same limit, and so is $K^{m k+2} u^{0}$, etc.

\section{Evolution problem with influencers}

We consider now the situation where $\Gamma \neq \emptyset$, i.e. some agents keep their initial opinion through the evolution process. Given $U \in \mathbb{R}^{\Gamma}$, that is values of the opinions of the influencers, we introduce the Dirichlet problem

$$
\left\{\begin{aligned}
u_{x}-\sum_{x \rightarrow y} K_{x y} u_{y} & =0 \quad \forall x \in \stackrel{\circ}{V} \\
u_{x} & =U_{x} \quad \forall x \in \Gamma .
\end{aligned}\right.
$$

This is a reformulation of the fixed point equation (3). The difference is that the value of $u$ 's on $\Gamma$ are thought as given, where those in $\stackrel{\circ}{V}$ are the unknowns.

We start by proving a static version of the maximum principle.

Proposition 1.9. (Maximum principle, static version)

Assume that $\Gamma \neq \emptyset$ and that any $x \in V$ is forwardly connected to $\Gamma$, i.e. $V-\cdot \rightarrow \Gamma$ (see Definition 1.1 ). Then for any $u$ solution to the Dirichlet problem (5), the maximum and the minimum of $u$ are attained on $\Gamma$.

Proof. Consider the point $x$ at which the maximum of $u$ is attained. Then for every $y$ connected to $x, u_{y}$ is also equal to the maximum. Progressing along the path which connectes $x$ to $\Gamma$, we obtain that the maximum is also realized at some point in $\Gamma$. Similarly, the minimum is attained on $\Gamma$.

Proposition 1.10. Assume that $\Gamma \neq \emptyset$ and that $V-\cdot \rightarrow \Gamma$. Then, for any given data $U$ on $\Gamma$, then the Dirichlet problem (5) admits a unique solution. Equivalently, the fixed point equation (3) admits a unique solution $u \in \mathbb{R}^{V}$ such that $u_{x}=U_{x}$ for all $x \in \Gamma$.

Proof. Let us first establish uniqueness. Consider two solutions associated to the same boundary data $U$. By linearity, the difference $u$ solves the same problem, with a zero data on the boundary. Since the maximum is attained on $\Gamma$, it is 0 , and so is the minimum. Therefore $u$ is identically 0 over $V$. Since the problem consists in solving a system of $N^{\circ}$ (number of inner vertices) equations with $N^{\circ}$ unknowns, uniqueness implies existence. Note that the rank-nullity theorem, which strongly uses the linear character of the evolution model, can be replaced by a more general topological argument. If one denotes by $\Lambda_{a}^{b} \subset \mathbb{R}^{N}$ the subset of all those vectors $u \in \mathbb{R}^{V}$ (identified to $\mathbb{R}^{N}$ ), with entries in the interval $[a, b]$, and such that $u$ identifies with $U$ on $\Gamma$, it is clear that the mapping

$$
K: u \in \mathbb{R}^{N} \longmapsto v \in \mathbb{R}^{N}, v_{x}=\sum_{x \rightarrow y} K_{x y} u_{y}
$$

is continuous and maps $\Lambda_{a}^{b}$ to itself. Since $\Lambda_{a}^{b}$ is convex and compact, Brouwer's Theorem (see for instance Theorem 6.6 in [17]) ensures existence of a fixed point.

Proposition 1.11. Under the assumptions of the previous proposition, for any initial condition $u^{0}$, the solution to the evolution problem converges to the unique equilibrium poin 3 , i.e. the unique solution to (5) when $U$ is the restriction of $u^{0}$ to $\Gamma$. 
Proof. Let $u^{e q}$ be the unique solution to (5) with boundary conditions given by $u_{\mid \Gamma}^{0}$. Let $\left(u^{k}\right)_{k}$ be the sequence defined as the solution to the evolution problem (2). Then the difference $w^{k}=u^{k}-u^{e q}$ satisfies the same evolution equation

$$
w_{x}^{k+1}=\sum_{x \rightarrow y} K_{x y} w_{y}^{k}
$$

and vanishes over $\Gamma$. By the maximum principle, $\max \left(w^{k}\right)$ decreases, and it is nonnegative (because of the boundary condition). It therefore converges to some limit $\ell \geq 0$. We aim at showing that $\ell=0$. If we are able to do that, it will be possible to show in the same way that $\min \left(w_{k}\right) \leq 0$ converges to 0 , so that $\left(w^{k}\right)$ converges to 0 , thus $\left(u^{k}\right)$ converges to $u^{e q}$.

Assume that $\ell>0$. Since the sequence $\left(w^{k}\right)$ is bounded, it admits a subsequence $w^{\varphi(k)}$ which converges to some $\bar{w} \in \mathbb{R}^{V}$, with $\max (\bar{w})=\ell$. Now consider the evolution problem associated to the initial state $\bar{w}$, we denote by $\left(\bar{w}^{k}\right)$ the corresponding sequence. We aim at showing that $\max \left(\bar{w}^{k}\right)$, which is $\ell$ for $k=0$, drops down to strictly smaller values after some time. To that purpose we define $X_{k}$ as the set of all those vertices $x$ which are in $\Gamma$, or connected to the boundary $\Gamma$ by a path made of vertices at which the value of $\bar{w}^{k}$ is (strictly) less than $\ell$. Consider a vertex $x$ at which $\bar{w}^{0}$ is $\ell$. This vertex is connected to the boundary by a path $x=x_{0}, \ldots$, $x_{n} \in \Gamma$. Now consider the largest integer $p$ such that $\bar{w}_{x_{p}}^{0}=\ell$. Since $x_{p}$ is connected to a vertex $x_{p+1}$ at which the value is less than $\ell$, its value will decrease to a smaller value, i.e. $\bar{w}_{x_{p}}^{1}<\ell$, so that $x_{p} \in \bar{X}_{1}$, while it was not in $X_{0}$. Since $X_{0} \subset X_{1}, X_{1}$ contains at least one vertex more than $X_{0}$. Similarly, as far as $X_{k} \neq V$, it is strictly included in $X_{k+1}$. Since the number of vertices is finite, there exists an index $m$ such that $X_{k}$ covers $V$ for $k \geq m$. As a consequence, $\max \left(\bar{w}_{m}\right)=\ell-\varepsilon$, for some $\varepsilon>0$

Now denote by $K^{m}$ the mapping which corresponds to $m$ iterations of the recurrence relation, so that $\bar{w}^{m}=K^{m} \bar{w}^{0}$. This mapping is continuous, and so is

$$
w \longmapsto \max \left(K^{m} w\right) .
$$

As a consequence, there exists $\beta>0$ such that, for any $w$ at a distance to $\bar{w}$ less than $\beta, \max \left(K^{m} w\right)<\ell-\varepsilon / 2$. Now since the sequence $w^{\varphi(k)}$ converges to $\bar{w}^{0}$,

$$
\exists L, \forall k \geq L,\left|w^{\varphi(k)}-\bar{w}^{0}\right|<\beta .
$$

Finally, for $k \geq L$,

$$
\max \left(K^{m} w^{\varphi(k)}\right)=\max \left(w^{\varphi(k)+m}\right)<\ell-\varepsilon / 2
$$

which is a contradiction because $\max \left(w^{k}\right) \geq \ell$ for any $k \in \mathbb{N}$.

In the case when some vertices are not connected to any influencer (i.e. there is no path toward a vertex in $\Gamma)$, the situation is more complicated. For instance, there might be cycles disconnected from $\Gamma$, in which the solution is periodic. Note that, on vertices which are both connected to $\Gamma$ and to such an autonomous cycle, no convergence to a stationary state can be expected, because of the periodic influence of the cycle (see the example of Figure 1).

Remark 1.12. (Lyapunov function)

The proof of Proposition (1.11) is an instantiation of a general approach to prove global asymptotic stability, based on the notion of Lyapunov function. Consider a dynamical system in $\mathbb{R}^{N}$ defined by $u^{k+1}=F\left(u^{k}\right)$, where $F$ is a continuous mapping. We assume that all trajectories are bounded, and that the system admits a unique equilibrium point, i.e. a unique $u^{e q} \in \mathbb{R}^{N}$ such that $u^{e q}=F\left(u^{e q}\right)$. Suppose now that there exists a continuous function $\Psi: \mathbb{R}^{N} \rightarrow \mathbb{R}$, which admits $u^{e q}$ as unique minimizer over $\mathbb{R}^{N}$. Assume furthermore that $F$ strictly decreases along the trajectories in the following sense: for any $u^{0} \in X \backslash\left\{u^{e q}\right\},\left(F\left(u^{k}\right)\right)$ is non-increasing, and there exists $k$ such that $F\left(u^{k}\right)<F\left(u^{0}\right)$. Then the sequence $\left(u^{k}\right)$ converges to $u^{e q}$ when $k$ goes to $+\infty$.

\footnotetext{
${ }^{3}$ Note that this equilibrium point depends on the initial condition, because the fixed values at the vertices of $\Gamma$ are those of the initial state.
} 


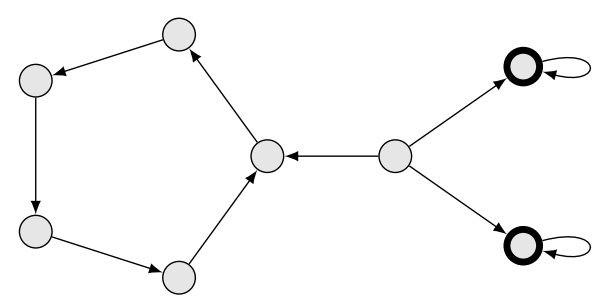

Figure 1. A node influence by $\Gamma$ (circled nodes) and by a cycle.

Proposition 1.13. (Uniform decay)

Under the assumptions of Proposition 1.10. let $u$ be the solution of the evolution problem (2) and $u^{e q}$ the equilibrium point to which it converges. There exists a sequence $\left(r_{k}\right)_{k \in \mathbb{N}}$ of real numbers tending to 0 , which depends only on the network (but not on $u^{0}$ ) such that for any $k$ and $x$,

$$
\left|u_{x}^{k}-u_{x}^{e q}\right| \leq r_{k}\left(\max _{y \in V}\left|u_{y}^{0}-u_{y}^{e q}\right|\right) .
$$

Proof. Let us start with the following observation. Let $v$ be a nonnegative solution of the evolution process (2), and $w$ a subsolution, i.e. a field in $\mathbb{R}^{V}$ such that, for every $k$ and $x$,

$$
w_{x}^{k+1} \leq \sum_{x \rightarrow y} K_{x y} w_{y}^{k}
$$

Then, provided that $w_{x}^{0} \leq v_{x}^{0}$ for every $x \in V$, a straightforward induction reasoning leads to $w_{x}^{k} \leq v_{x}^{k}$ for any $k$ and $x$. Now consider $w_{x}^{k}=\left|u_{x}^{k}-u_{x}^{e q}\right|$. It holds that

$$
w_{x}^{k+1}=\left|u_{x}^{k+1}-u_{x}^{e q}\right|=\left|\sum_{x \rightarrow y} K_{x y}\left(u_{x}^{y}-u_{y}^{e q}\right)\right| \leq \sum_{x \rightarrow y} K_{x y}\left|u_{x}^{y}-u_{y}^{e q}\right|=\sum_{x \rightarrow y} K_{x y} w_{x}^{k},
$$

so that $w$ is a subsolution. We now define $\hat{v}^{k}$ as the solution of 2 with initial conditions given by

$$
\hat{v}_{x}^{0}= \begin{cases}1 & \text { if } x \in \stackrel{\circ}{V}=V \backslash \Gamma, \\ 0 & \text { if } x \in \Gamma\end{cases}
$$

and $v_{x}^{k}=\left(\max _{y} w_{y}^{0}\right) \hat{v}_{x}^{k}$. By linearity $v$ is a solution of the evolution process 2 with $w_{x}^{0} \leq v_{x}^{0}$ for every $x$. Thanks to the observation above, we conclude that $0 \leq w_{x}^{k} \leq v_{x}^{k}$ for every $k, x$.

Thanks to Proposition 1.11, we know that $\hat{v}^{k}$ converges to a fixed point, which is the uniform field equal to 0 given the values of $\hat{v}^{0}$ on $\Gamma$. Moreover $\hat{v}$ does not depend on $w$ but only on the network. Calling $r_{k}=\max _{y} \hat{v}_{y}^{k}$, we reach the conclusion.

Remark 1.14. Using spectral theory, one could expect to bound $r_{k}$ by $C e^{-c k}$, where $c$ is related to the spectrum of $K$. However, if $K$ is not normal (diagonalizable in $\mathbb{C}$ in a Hermitian basis), the constant $C$ in front of the exponential can be very large, hence this kind of upper bound might be of little value.

\section{Stochastic Setting}

We describe here a stochastic interpretation of the so-called Dirichlet problem (3) studied in the previous section. We consider the situation where $\Gamma \neq \emptyset$, and $V-\cdot \rightarrow \Gamma$ (see Def. 1.1). We denote by $U$ a given boundary field, defined on $\Gamma$. 
A random walk 4 can be canonically defined on the weighted graph $(V, E, K)$, with a transition probability of hopping from $x$ to $y$ equal to $K_{x y}$. From any $x$, we consider a random walk starting from $x$, according to the transition probabilities $\left(K_{x y}\right)$, and we denote by $j$ the index which corresponds to the first hit with $\Gamma$ :

$$
X_{0}=x, X_{1}, \ldots, X_{j} \in \Gamma, X_{i} \notin \Gamma \quad \forall i<j .
$$

The value of $U$ at $X_{j}$ is a random variable associated to the starting point $x$. We denote its expected value by $u_{x}$.

Proposition 2.1. The field $u \in \mathbb{R}^{V}$ defined above is a solution to the Dirichlet problem (5), which writes

$$
\left\{\begin{aligned}
(I-K) u & =0 \\
u_{x} & =U_{x} \quad \forall x \in \Gamma
\end{aligned}\right.
$$

where $(I-K) u=0$ is the matrix formulation of

$$
u_{x}-\sum_{x \rightarrow y} K_{x y} u_{y}=0
$$

Proof. Let us first notice that $u$ identifies to $U$ on $\Gamma$ (when $x \in \Gamma$ then $j=0$ ). The rest simply comes from a local expression of conditional expectations: since, starting from $x, K_{x y}$ is the probability to go to $y$, it holds that

$$
u_{x}=\sum_{x \rightarrow y} K_{x y} u_{y}
$$

i.e. $u$ verifies $(3)$.

Let us denote by $\pi_{x}^{k}$ the probability that the particle lies at vertex $x$ at step $k$. The probability field verifies the following relation

$$
\pi_{x}^{k+1}=\sum_{y \rightarrow x} K_{y x} \pi_{y}^{k} \quad \text { whereas } \quad u_{x}^{k+1}=\sum_{x \rightarrow y} K_{x y} u_{y}^{k}
$$

Note the difference with the evolution equation for $u^{k}$ : the propagation occurs in the reverse direction. The two evolution problems are mutually adjoints: the first one (equation on $\pi$ associated to $K^{T}$ ) involves a probability measure, and thus enjoys a conservation property. Indeed,

$$
\sum_{x} \pi_{x}^{k+1}=\sum_{x} \sum_{y \rightarrow x} K_{y x} \pi_{y}^{k}=\sum_{y} \pi_{y}^{k} \underbrace{\sum_{y \rightarrow x} K_{y x}}_{=1}=\sum_{y} \pi_{y}^{k} .
$$

The second one (equation on $u$ associated to $K$ ) enjoys a maximum principle property, by Proposition 1.4 It suggests a natural framework in terms of norms for those two matrices seen as operators on Banach spaces: the natural norm for $u$ is $\ell^{\infty}$, whereas the natural norm for $\pi$ is $\ell^{1}$, as illustrated by the diagram:

$$
\begin{array}{lll}
\left(\mathbb{R}^{N}, \ell^{\infty}\right) \stackrel{K}{\longleftrightarrow}\left(\mathbb{R}^{N}, \ell^{\infty}\right) \\
\left(\mathbb{R}^{N}, \ell^{1}\right) \stackrel{K^{T}}{\longleftarrow}\left(\mathbb{R}^{N}, \ell^{1}\right),
\end{array}
$$

and the straightforward properties

$$
\|K\|_{\infty}=\sup _{\mathbb{R}^{N}} \frac{\|K u\|_{\infty}}{\|u\|_{\infty}}=1=\sup _{\mathbb{R}^{N}} \frac{\left\|K^{T} \pi\right\|_{1}}{\|\pi\|_{1}}=\left\|K^{T}\right\|_{1} .
$$

\footnotetext{
${ }^{4}$ This section is based on some basic properties of random walks, all of them are quite intuitive and not central in these notes, so that we chose to alleviate the formalism of stochastic processes and rely on the reader's common sense. We refer to 18 . for a rigorous presentation of Markov chains.
} 
From this standpoint, maximum principle and mass conservation can be seen as mutually adjoint properties. We shall consider in Section 5 the situation where the matrices are self-adjoint in some sense (i.e. for some scalar product), which then provides the evolution problem with both maximum principle and mass conservation (in a certain sense, that is preservation of the expected value with respect to the stationary measure, or charisma, see Equation (19)).

The probabilistic interpretation leads to the introduction of objects called harmonic measures.

Definition 2.2. (Harmonic measure)

Let $x_{0} \in V$ a vertex of $V$. The harmonic measure with pole at $x_{0}$, denoted by $\mu_{x_{0}, \cdot}$, is a measure on $\Gamma$ such that $\mu_{x_{0}, y}$ is the probability for a random walk starting from $x_{0}$ and with probability transitions given by $K$ to hit the set $\Gamma$ at $y$. Since we the random walk will (almost surely) hit the boundary after some time (see [18]), it holds that that $\sum_{y \in \Gamma} \mu_{x_{0}, y}=1$, i.e. it is a probability measure. This measure can be considered as a measure over $V$ by simply setting $\mu_{x_{0}, y}=0$ for $y \notin \Gamma$.

Proposition 2.3. Let $U \in \mathbb{R}^{\Gamma}$ and $u \in \mathbb{R}^{V}$ the unique solution of the associated Dirichlet problem (see Proposition 1.10). Then, for any $x_{0} \in V$,

$$
u_{x_{0}}=\sum_{y \in \Gamma} \mu_{x_{0}, y} U_{y}
$$

where $\mu_{x_{0}, y}$ is the harmonic measure with pole at $x_{0}$ (Definition 2.2).

Proof. We simply use Proposition 2.1. We know that $u_{x_{0}}$ is the expectation of $U_{Y}$, where $Y$ is the point of $\Gamma$ hit by a random walk issued from $x_{0}$. Hence,

$$
u_{x_{0}}=\mathbb{E}\left[U_{Y}\right]=\sum_{y \in \Gamma} \mathbb{E}\left[U_{Y} 1_{\{Y=y\}}\right]=\sum_{y \in \Gamma} U_{y} \underbrace{P(Y=y)}_{=\mu_{x_{0}, y}} .
$$

The value $u_{x_{0}}$ is thus a convex combination of the values of $U$, and the coefficients of this convex combination are given by the coefficients $\left(\mu_{x_{0}, y}\right)_{y \in \Gamma}$. In particular, $\mu_{x_{0}, y}$ is the equilibrium opinion at $x_{0}$ associated to the following boundary data: $U=\delta_{y}$, i.e. $y$ thinks 1 , and all other influencers think 0 .

Proposition 2.4. Let $x_{0}$ be given and let us consider the linear problem

$$
\left\{\begin{aligned}
\left(\nu-K^{T} \nu\right)_{x} & =\delta_{x_{0}, x} & & \text { for all } x \in \stackrel{\circ}{V}=V \backslash \Gamma, \\
\nu_{y} & =0 & & \text { for } y \in \Gamma .
\end{aligned}\right.
$$

where $\delta_{., .}$is the Kronecker delta, i.e. $\delta_{x_{0}, x}=1$ if $x=x_{0}$, and 0 otherwise.

This problem admits a unique solution. Moreover, if we call $\nu$ this unique solution, it holds that

$$
\mu_{x_{0}, y}=\left(K^{T} \nu-\nu\right)_{y}=\sum_{x \rightarrow y} K_{x y} \nu_{x} \quad \forall y \in \Gamma .
$$

Proof. We briefly say a word about existence and uniqueness. In fact, the problem defining $\nu$ is a linear system, the matrix of which is the transpose of the one involved in Dirichlet Problem (5). Thanks to Proposition 1.10. we know that the latter problem is well-posed, i.e the matrix is nonsingular.

We fix $y_{0} \in \Gamma$. Let $u$ be the unique solution of the following problem:

$$
\left\{\begin{aligned}
(u-K u)_{x}=0 & \text { for all } x \in \stackrel{\circ}{V}, \\
u_{y_{0}}=1, & \text { for } y \in \Gamma, y \neq y_{0} .
\end{aligned}\right.
$$


Actually, $u$ is nothing else than the fixed point given by Proposition 1.10 if we take $\delta_{y_{0}}$. as boundary data. In particular, $\mu_{x_{0}, y}=u_{x_{0}}$. On the other hand, from the definition of the transposition, we have that

$$
\sum_{(x, y) \in V^{2}}\left(u_{x}-K_{x y} u_{y}\right) \nu_{x}=\langle u-K u \mid \nu\rangle=\left\langle u \mid \nu-K^{T} \nu\right\rangle=\sum_{(x, y) \in V^{2}} u_{x}\left(K_{y x} \nu_{x}-\nu_{y}\right) .
$$

By the definitions of $u$ and $\nu$, the left hand side vanishes, so that

$$
0=u_{x_{0}}-u_{y_{0}}\left(K^{T} \nu-\nu\right)_{y_{0}}=\mu_{x_{0}, y_{0}}-\left(K^{T} \nu-\nu\right)_{y_{0}}
$$

which ends the proof.

We refer to Section 3 (see Remark 3.2 for an alternative standpoint on this harmonic measure, namely: $u$ being the solution to (6) for given boundary data $U, \mu_{x_{0}, y_{0}}$ expresses the dependence of the opinion at $x_{0}$ upon $y_{0}$ 's opinion, more precisely it is the partial derivative of $u_{x_{0}}$ with respect to $U_{y_{0}}$.

Remark 2.5. Proposition 2.4 is a discrete analog, in the non-symmetric setting, of a general property on the so called harmonic measure associated to a Euclidean domain (see e.g. [27], p. 651). Consider a smooth domain $\Omega \subset \mathbb{R}^{d}$ with boundary $\Gamma=\partial \Omega, x_{0} \in \Omega$, and denote by $\nu$ the solution to the Poisson problem

$$
\left\{\begin{array}{rlll}
-\Delta \nu & =\delta_{x_{0}} & & \text { in } \Omega \\
\nu & =0 & & \text { on } \Gamma
\end{array}\right.
$$

The field $-\partial \nu / \partial n$ defined on $\partial \Omega$, which is the continuous counterpart of $(10)$, is also the probability density for the location of the first hit of the boundary $\partial \Omega$ by a Brownian motion issued from $x_{0}$. The Dirichlet problem above is a continuous counterpart of the discrete problem (9). In the same spirit, Proposition 2.1 is the discrete counterpart of a standard property in potential theory. Let us consider a smooth scalar field $U$ defined on the boundary $\Gamma$, and the associated Laplace problem

$$
\left\{\begin{array}{rlll}
-\Delta u & = & & \text { in } \Omega \\
u & = & U & \text { on } \Gamma
\end{array}\right.
$$

that is the continuous counterpart of Problem (6). For any $x \in \Omega$, consider the Brownian motion issued from $x$, denote by $X \in \Gamma$ the location of its first contact with $\Gamma$. The expected value of $U(X)$ is $u(x)$, where $u$ is the solution to the Laplace problem above (see again [27], p. 651). Note that, in the discrete, non-symmetric setting, the two problems are natively set on different spaces in terms of norms: Problem $(6)$ is set in $\left(\mathbb{R}^{N}, \ell^{\infty}\right)$, whereas Problem $(9)$ is set in $\left(\mathbb{R}^{N}, \ell^{1}\right)$ (see Diagram (8)).

\section{Monitoring of A network though influencers / Influence COEFficients}

We describe in this section how the so-called adjoint method can be carried out to investigate the dependence of some global quantities upon some parameters, called in this context control variables. We shall consider here two options for the choice of control variables: opinions of influencers, and influence coefficients $K_{x y}$. We consider as previously a set $V$ of agent, a collection of influence coefficients $\left(K_{x y}\right)$ encoded in a stochastic matrix $K$. We recall that influencers are agents $x$ such that $K_{x x}=1$, that $\Gamma$ denotes the set of influencers, and that $\stackrel{\circ}{V}=V \backslash \Gamma$ is the set of interior vertices. We assume that $V-\cdot \rightarrow \Gamma$, i.e. each vertex is connected by a path to the boundary (see Definition 1.1).

The core of the approach is the so-called state equation, which is in our case the static Dirichlet problem (5). We shall reformulate it as a linear system on the unknowns associated to the interior vertices. We denote by $U \in \mathbb{R}^{\Gamma}$ the collection of influencers' opinions, and by $\stackrel{\leftrightarrow}{u} \in \mathbb{R}^{V}$ the collection of unknowns (opinions at interior nodes), so that $u=(\stackrel{\circ}{u}, U) \in \mathbb{R}^{V}$ is the full collection of opinions. The matrix formulation of $(5)$ is $A u=0$, with 
$A=I-K$, and $u_{x}=U_{x}$ for any $x \in \Gamma$. It will be more convenient here to separate interior degrees of freedom and influencers' opinions. The problem can be written

$$
\stackrel{\circ}{u}_{x}-\sum_{x \rightarrow y \in \dot{V}} K_{x y} \stackrel{\circ}{u}_{y}=\sum_{x \rightarrow y \in \Gamma} K_{x y} U_{y} \quad \forall x \in \stackrel{\circ}{V},
$$

which can be written in a matrix form

$$
\stackrel{\circ}{\mathrm{u}}=-K_{\Gamma} U,
$$

where $\AA$ is a submatrix of $A$ (restriction to interior degrees of freedom). The matrix $K_{\Gamma}$, which encodes a mapping from $\mathbb{R}^{\Gamma}$ to $\mathbb{R}^{V}$, integrates the effect of influencers' opinions. For any $U \in \mathbb{R}^{\Gamma}$, Problem (11) admits a unique solution (Proposition 1.10, which we denote by $\stackrel{\circ}{U}_{U}$. We introduce the so-called cost function ${ }^{5}$, that is a function of $U$ given as an expression of $\stackrel{\leftrightarrow}{u}_{U}$, i.e.

$$
J(U)=\Phi\left(\stackrel{\circ}{u}_{U}\right),
$$

where $\Phi(\stackrel{\circ}{)}$ is typically a function which one aims at minimizing. To fix the idea, we may consider that $\Phi$ is defined as

$$
\Phi\left(\stackrel{\imath}{)}=\frac{1}{2}|\stackrel{\leftrightarrow}{u}-\hat{u}|^{2},\right.
$$

where $\hat{u} \in \mathbb{R}^{\stackrel{\circ}{ }}$ is a targeted collection of opinions. Minimizing $\Phi\left(\stackrel{\circ}{u}_{U}\right)$ with respect to $U$ consists in finding the collection of influencers' opinions such that the associated opinions are the closest to $\hat{u}$. An essential ingredient to actually perform this minimization is the gradient of this functional with respect to $U$. We shall now describe a classical method to estimate this gradient. Note that, beyond the actual use of this gradient in a minimization procedure, it can be also useful in terms of modeling to actually estimate the sensitivity of a functional (possibly the opinion of a specific agent, see Remak 3.2, with respect to influencers' opinions, or any other parameter.

\section{Description of the adjoint method}

The approach is based on a so-called Lagrangian $L$ defined as

$$
L:(\stackrel{\circ}{u}, U, p) \in \mathbb{R}^{\stackrel{\circ}{V}} \times \mathbb{R}^{\Gamma} \times \mathbb{R}^{\stackrel{\circ}{ }} \longmapsto \Phi(\stackrel{\circ}{u})+\left\langle\stackrel{\circ}{\mathrm{u}}-K_{\Gamma} U \mid p\right\rangle .
$$

It holds that, for any $p \in \mathbb{R}^{V^{\circ}}$,

$$
J(U)=L\left(\stackrel{\circ}{u}_{U}, U, p\right)
$$

As a consequence

$$
D J(U)=D_{\grave{u}} L\left(\stackrel{\circ}{u}_{U}, U, p\right) \circ D_{U} \stackrel{\circ}{u}_{U}+D_{U} L\left(\stackrel{\circ}{u}_{U}, U, p\right) .
$$

(We underline here that $D_{U} L\left(\stackrel{\varkappa}{u}_{U}, U, p\right)$ stands for the partial derivative of $L(\cdot, \cdot, \cdot)$ with respect to the second variable $U$, taken at $\left(\stackrel{\circ}{u}_{U}, U, p\right), \stackrel{\circ}{u}_{U}$ being frozen at its current value.)

The strategy consists in building a $p$ such that $D_{\mathfrak{u}} L\left(\stackrel{\circ}{u}_{U}, U, p\right)=0$ in the expression above. For such a $p$, $D J(U)$ is simply $D_{U} L$, which depends on this very $p$. From the expression of the Lagrangian, we have that ${ }^{6}$

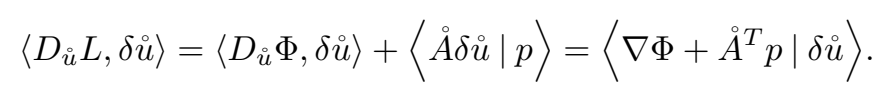

The adjoint problem for the dual variable $p$ is defined in order to vanish the quantity above. The problem writes

$$
\AA^{T} p=-\nabla \Phi
$$

\footnotetext{
${ }^{5}$ Also called loss or objective function in the context of Machine Learning (see e.g. 39]).

${ }^{6}$ We use the notation $\langle\cdot, \cdot\rangle$ to represent the duality pairing between the differential of a mapping and a variation of the considered variable, whereas $\langle\cdot \mid \cdot\rangle$ still represents the scalar product between two elements of the same Euclidean space, $\mathbb{R}^{{ }^{V}}$ or $\mathbb{R}^{\Gamma}$.
} 
Let $p$ be the solution to this problem, it holds that

$$
\langle D J(U), \delta U\rangle=\left\langle D_{U} L\left(\stackrel{\circ}{u}_{U}, U, p\right), \delta U\right\rangle=-\left\langle K_{\Gamma} \delta U \mid p\right\rangle=-\left\langle K_{\Gamma}^{T} p \mid \delta U\right\rangle .
$$

As a consequence, $\nabla J(U)=-K_{\Gamma}^{T} p$, where $p$ is the solution to the adjoint problem (13).

In the case of a cost function defined by $(12)$, the right-hand side of $(13)$ is $\hat{u}-\stackrel{\circ}{u}_{U}$. In the case of a cost function that is the value of $u$ at some point $x_{0}$, the right-hand side is the discrete Dirac mass $-\delta_{x_{0}}($ see Remark 3.2 below).

Remark 3.1. The adjoint problem can be interpreted as a discrete Laplace problem (based on the matrix adjoint to the non-symmetric Laplacian $I-K)$, with homogeneous Dirichlet boundary conditions. Indeed, Problem (13) expresses

$$
p_{y}-\sum_{y \leftarrow x \in \dot{\circ}} K_{y x} p_{x}=(\nabla \Phi)_{x} \quad \forall y \in \stackrel{\circ}{V}
$$

and the sum over vertices can be extended to $V$ if one sets $p_{x}$ to 0 for any $x \in \Gamma$.

Remark 3.2. This approach makes it possible to recover the notion of harmonic measure introduced in Section 2. The idea consists in choosing another functional, that is $\Phi(u)=u_{x_{0}}$ for a certain $x_{0} \in \stackrel{\circ}{V}$. The adjoint problem reads $\AA^{T} p=-\delta_{x_{0}}$, which is another way to express $\sqrt{9}$. The gradient of $J(U)=\left(u_{U}\right)_{x_{0}}$ is then a vector defined on $\Gamma$, the entries of which are the weights of the opinions of the various influencers upon $x_{0}$, i.e. the values of the harmonic measure $\mu_{x_{0}, y}$, for $y \in \Gamma$.

\section{Effective minimization of the cost functional}

Considering a quadratic functional of the type $J(U)=\Phi\left(\stackrel{\circ}{u}_{U}\right)$, where $\Phi$ is given by $(12)$, minimizing $J(U)$ amount to find a $U \in \mathbb{R}^{\Gamma}$ at which the gradient is 0 . A straightforward computation leads to the following matrix formulation of this condition:

$$
\left(\stackrel{\circ}{A}^{-1} K_{\Gamma}\right)^{T} \stackrel{\circ}{A}^{-1} K_{\Gamma} U=-\left(\stackrel{\circ}{ }^{-1} K_{\Gamma}\right)^{T} \hat{u} .
$$

Note that the matrix $S=\left(\AA^{-1} K_{\Gamma}\right)^{T} \AA^{-1} K_{\Gamma} U$ is symmetric definite positive. Indeed, it is of the form $B^{T} B$, therefore symmetric and nonnegative. Besides, $S U=0$ implies $B U=0$, which means that the solution to Problem (11) (which is another way to write Dirichlet problem (5)) is zero, so that $U=0$. A Conjugate Gradient Method can then be implemented to solve this problem.

\section{Derivative with respect to influence coefficients}

We consider now the problem of determining the derivative of some function of the interior opinions with respect to the influence coefficients $K_{x y}$. This field can be identified to a vector in $\mathbb{R}^{E}$, where $E=\operatorname{supp}\left(K_{x y}\right)$ is the set of edges. We shall assume that influencers remain influencers, i.e. $K_{y y}$ remains equal to 1 for any $y \in \Gamma$, and variations are restricted to connections between interior vertices and their neighbors (possibly influencers). We shall consider variations of coefficients on existing edges, but the approach may be extended to variations on all possible links.

Since the vector of influencers' opinions is no longer a variable, we shall drop the dependence of $\stackrel{i}{u p o n} U$, and replace it by a dependence upon $K$. We write the state equation as

$$
\stackrel{\circ}{A}_{K} \stackrel{\circ}{u}_{K}=b_{K}, \quad \text { with } b_{K}=K_{\Gamma} U \in \mathbb{R}^{V}, \quad \text { i.e. }\left(b_{K}\right)_{x}=\sum_{x \rightarrow y \in \Gamma} K_{x y} U_{y} \quad \forall x \in \stackrel{\circ}{V}, x \rightarrow \Gamma,
$$

with $\left(b_{K}\right)_{x}=0$ for any all vertices which are not directly connected to $\Gamma$. The Lagrangian now writes

$$
L:(\stackrel{\circ}{u}, K, p) \in \mathbb{R}^{\stackrel{\circ}{V}} \times \mathbb{R}^{E} \times \mathbb{R}^{\stackrel{\circ}{V}} \longmapsto \Phi(\stackrel{\circ}{u})+\left\langle\stackrel{\circ}{A}_{K} \stackrel{\circ}{u}-b_{K} \mid p\right\rangle .
$$


It can be used, as previously, to estimate the gradient of $K \mapsto J(K)=\Phi\left(\stackrel{\circ}{u}_{K}\right)$. The adjoint problem is the same as previously, i.e. Equation (13). It remains to estimate the derivative of $L$ with respect to $K$. The part of $L$ which depends on $K$ writes

$$
\sum_{x \in \dot{V}} p_{x} \sum_{x \rightarrow y} K_{x y}\left(u_{x}-u_{y}\right)=\sum_{e \in E}\left(\sum_{x \rightarrow y} p_{x}\left(u_{x}-u_{y}\right)\right) K_{x y}
$$

where $u_{x}$ is $U_{x}$ whenever $x \in \Gamma$, and $\stackrel{\circ}{E}$ is the set of edges except influencers' loops. We finally obtain

$$
\nabla J(K)=\left(p_{x}\left(u_{x}-u_{y}\right)\right)_{(x, y) \in \stackrel{\circ}{ }}
$$

where $p$ is the solution to the adjoint problem 13).

N.B. : Implementing this approach to actually minimize the cost function necessitates to account for the constraints on the $K_{x y}$ 's: linear constraints on the sums of coefficients on each row, and unilateral constraints $K_{x y} \in[0,1]$. Notice also that the problem is quite different from the previous one, with influencers' opinions as control variables, because the mapping $K \longmapsto u_{K}$ is not linear, and consequently the global functional $J(\cdot)$ is not quadratic.

\section{From Discrete to CONTINUOUS IN TIME PROBLEM}

The fact that it takes some time for $x$ to integrate the influence of their neighbors can be modelled by introducing a parameter $\theta \in[0,1]$, and write the relaxed problem

$$
u_{x}^{k+1}=(1-\theta) u_{x}^{k}+\theta \sum_{x \rightarrow y} K_{x y} u_{y}^{k}
$$

For $\theta=1$, we recover the initial problem. Note that this new problem actually fits in the previous frameworks, by considering modified weights

$$
K_{x x}^{\prime}=(1-\theta)+\theta K_{x x}, K_{x y}^{\prime}=\theta K_{x y} \text { for } y \neq x .
$$

Remark 4.1. The relaxation by $\theta \in[0,1)$ is likely to change the behavior of the solution. In particular, if the graph is strongly connected, then adding a loop from any vertex to itself makes it such that there exists an integer $m$ such that any two vertices $x$ and $y$ can be connected by a path of length $m$. This feature is quite important in terms of asymptotic behavior, as expressed by Corollary 1.8.

\section{Differential equation}

Consider now that $\theta$ writes as $\varepsilon / \eta$, where $\eta$ is a fixed relaxation time, and $\varepsilon$ a small parameter (also homogeneous to a time). It holds that

$$
\frac{u_{x}^{k+1}-u_{x}^{k}}{\varepsilon}=\frac{1}{\eta}\left(\sum_{x \rightarrow y} K_{x y}\left(u_{y}^{k}-u_{x}^{k}\right)\right) .
$$

It takes the form of the time discretization of a system ordinary differential equations for quantities $t \mapsto u_{x}^{t} \in \mathbb{R}$, which continuously vary in time, and the underlying equation is

$$
\frac{d}{d t} u_{x}^{t}=\frac{1}{\eta}\left(\sum_{x \rightarrow y} K_{x y}\left(u_{y}^{t}-u_{x}^{t}\right)\right) .
$$


The time continuous problem thus takes the form

$$
\frac{d}{d t} u^{t}=-\frac{1}{\eta} A u^{t} \quad \text { with } \quad A=I-K .
$$

\section{Spectral stability}

Thanks to Gershgorin theorem, the spectrum of $K$ lies in the union of discs $D_{x} \in \mathbb{C}$, centered at $K_{x x}$ with a radius equal to the sum of extradiagonal entries: defined by

$$
S_{p}(K) \subset \bigcup_{x \in V} D_{x}, D_{x}=\left\{z \in \mathbb{C},\left|z-K_{x x}\right| \leq \sum_{y \neq x} K_{x y}\right\}
$$

Since $K_{x y} \in[0,1]$ and $\sum_{y} K_{x y}=1$, all the $D_{x}$ 's are contained in the unit disc, so that the spectrum of $A=I-K$ is contained in the disc of center 1 and radius 1 . Notice that, since $K$ is stochastic, $K e=e$, with $e=(1,1, \ldots, 1)$. This matrix therefore admits 1 as an eigenvalue (possibly with multiplicity), so that the corresponding value 0 is an eigenvalue for $A$. As a consequence, the eigenvalues of $A$ have a positive real part, (aside from 0 ), so that the linear system is stable.

\section{Gradient flow structure}

We aim here at characterizing the case when the evolution problem 15 has a gradient flow structure for some scalar product.

For any symmetric positive definite matrix $M$, we denote by $\langle\cdot \mid \cdot\rangle_{M}$ the associated scalar product, i.e.

$$
\langle u \mid v\rangle_{M}=\langle M u \mid v\rangle
$$

For any $C^{1}$ functionnal $\Phi: \mathbb{R}^{N} \rightarrow \mathbb{R}$, we denote by $\nabla_{M} \Phi(u) \in \mathbb{R}^{N}$ its gradient at $u$ according to the scalar product associated to $M$, i.e. the only vector such that

$$
\Phi(u+h)=\Phi(u)+\left\langle\nabla_{M} \Phi \mid h\right\rangle_{M}+o(h) .
$$

Now assume that $\Phi$ is a quadratic functional, i.e. $\Phi(v)=\langle B v \mid v\rangle / 2$ for some matrix $B$. Since $\langle C v \mid v\rangle=$ $\left\langle v \mid C^{T} v\right\rangle$ vanishes if $C$ is skew-symmetric, the functional only depends on the symmetric part of $B$, hence we may assume that $B$ is symmetric. We have

$$
\Phi(u+h)=\Phi(u)+\langle B u \mid h\rangle+o(h)=\Phi(u)+\left\langle M^{-1} B u \mid h\right\rangle_{M}+o(h),
$$

so that $\nabla \Phi(u)=B u$, and $\nabla_{M} \Phi=M^{-1} B u$. As a consequence, $A u$ is the gradient of a quadratic functional if and only if $A$ is symmetric, and it is the gradient of a quadratic functional according to some scalar product associated to the s.p.d. matrix $M$ if and only if $A=M^{-1} B$ for some symmetric matrix $B$.

In Section 5 , we will concentrate of a particular type of networks which carry a gradient flow structure, namely charismatic network (see Definition 5.1). For those networks, we shall have $K=M^{-1} B$, where $M$ is diagonal, with diagonal coefficients corresponding to the charisma $\left(\omega_{x}\right)_{x \in V}$ of agents, and $B$ defined by $B_{x y}=\omega_{x} K_{x y}$ is symmetric. It corresponds to the case where the underlying Markov chain is reversible. More generally, we can give a more explicit characterization of all those matrices for which Equation 15 has a gradient flow structure (with a possibly non-diagonal matrix $M$ ).

Proposition 4.2. The evolution problem 15) has a gradient flow structure for some scalar product if and only if $A$ (or, equivalently, $K$ ) is diagonalizable, and all its eigenvalues are real. 
Proof. If $A u$ is the gradient of a quadratic functional at $u$ for some scalar product $\langle\cdot \mid \cdot\rangle_{M}$, then there exists a symmetric matrix $B$ such that $A=M^{-1} B$. Since $M$ is s.d.p., it writes $M=U D U^{-1}$, where $U$ is an orthonormal matrix and $D$ is diagonal. We define $M^{1 / 2}$ as $U D^{1 / 2} U^{-1}$. Then $A$ is similar to

$$
M^{1 / 2} A M^{-1 / 2}=M^{1 / 2} M^{-1} B M^{-1 / 2}=M^{-1 / 2} B M^{-1 / 2},
$$

which is symmetric, since $B$ is. As a consequence, $A$ is similar to a symmetric matrix, therefore it is diagonalizable with real eigenvalues.

Now assume that $A$ has only real eigenvalues: $A=P D P^{-1}$ where $D$ is diagonal, with real entries, and $P$ is a nonsingular matrix. We consider the scalar product associated to the s.d.p. matrix $M=P^{-T} P^{-1}$ :

$$
\langle u \mid v\rangle_{M}=\left\langle P^{-1} u \mid P^{-1} v\right\rangle=\left\langle P^{-T} P^{-1} u \mid v\right\rangle .
$$

It is a scalar product for which the columns of $P$ form an orthonormal basis. The matrix $A$ can be written

$$
A=P D P^{-1}=P P^{T} P^{-T} D P^{-1}=M^{-1} P^{-T} D P^{-1}=M^{-1} B
$$

where $B=P^{-T} D P^{-1}$ is a symmetric real matrix.

Remark 4.3. In the gradient flow case, $A$ is a maximal monotone operator in $\mathbb{R}^{V}$ (see e.g. 19 ) for a certain scalar product. Indeed, as seen in the proof of Proposition 4.2, $A=I-K$ writes $P D P^{-1}$ where $D$ is diagonal, with real nonnegative entries, and $P$ is a nonsingular matrix. Now $M=P^{-T} P^{-1}$ defines a scalar product for which

$$
\langle A v \mid v\rangle_{M}=\langle M A v \mid v\rangle=\left\langle P^{-T} P^{-1} P D P^{-1} v \mid v\right\rangle=\left\langle D P^{-1} v \mid P^{-1} v\right\rangle \geq 0 .
$$

Remark 4.4. With the help of this proposition, we deduce that the set of networks leading to an evolution problem having a gradient flow structure is generically stable under small perturbations. Indeed, let us denote by $\mathcal{G} \subset \mathbb{R}^{V \times V}$ the set of matrices which are diagonalizable with only real eigenvalues, and by $\mathcal{D} \subset \mathbb{R}^{V \times V}$ the set of matrices which are diagonalizable with real and distinct eigenvalues. The latter set, which corresponds to the one of matrices whose characteristic polynomial has only distinct real roots, is open in $\mathbb{R}^{V \times V}$ and clearly included in $\mathcal{G}$, with dense inclusion and full measure. On other other hand, each element of $\mathcal{G}$ belongs to $\overline{\mathcal{D}}$ : any diagonalizable matrix can be slightly perturb so that all its eigenvalues are distinct. In short,

$$
\mathcal{D} \subset \mathcal{G} \subset \overline{\mathcal{D}}
$$

with strict and dense inclusions. As a consequence, a generic matrix in $\mathcal{G}$, i.e. a matrix which does not belong to $\mathcal{G} \backslash \mathcal{D} \subset \partial \mathcal{D}$, belongs to the interior of $\mathcal{G}$, and therefore remains of the gradient type under small pertubations.

\section{Typical non-gradient like situations}

As mentioned previously, the gradient flow character is generic and structurally stable in some regions of the set of stochastic matrices. Yet, in general, the evolution problem (15) is not a gradient flow, and we shall describe here two typical non-gradient situations: when the matrix has only real eigenvalues but is not diagonalizable, and when the matrix is diagonalizable with non-real eigenvalues.

\section{Hierarchical graph and non-diagonalizable matrices}

We consider here acyclic graphs, i.e. graphs which do not contain cycles (see Definition 1.1). Such graphs are called hierarchical, because it can be shown that there exists an ordering $x_{1}, x_{2}, \ldots, x_{N}$ of the vertices in such a way that $x_{i} \rightarrow x_{j}$ implies $i \leq j$. Such an indexing, which is obviously not unique in general, can be built by mean of topological sort (see e.g. 22]). To simplify the situation, we shall assume that $K_{x x}>0$ only for the influencers (and in this case $K_{x x}=1$ ). 
With these assumptions, under the chosen ordering of the vertices, the matrix $K$ is upper-triangular, with only 1 and 0 on the diagonal:

$$
K=\left(\begin{array}{c|c}
B & H \\
\hline 0 & I_{m}
\end{array}\right),
$$

where $m$ is the number of influencers, $I_{m}$ is the identity matrix of size $m, B$ is a $(N-m) \times(N-m)$ upper triangular matrix with only zeros on the diagonal, and $H$ is a $(N-m) \times m$ matrix. Note that $B$ is nilpotent. The matrix $K$ has only two eigenvalues, namely 1 and 0 , but the kernel of $K$, i.e. the eigenspace associated to the eigenvalue 0 , has a dimension strictly smaller than $N-m$. Note that, in the archetypal situation of a linear network $x_{1} \rightarrow x_{2} \rightarrow \cdots \rightarrow x_{N} \rightarrow x_{N}$, we have that $m=1, B$ is the $(N-1)$ square matrix with only zeros except for the first upper diagonal filled with 1 , and $H$ is a column matrix filled with 0 , except for the last entry which is 1 . The matrix $K$ then natively identifies with its Jordan normal form.

Let us first consider the discrete model, i.e. the iterative process process (2). After at most $N-m$ iterations, the fixed point state will be reached and the opinion of every agent but the influencer will have been flushed out of the network. Indeed, as $B^{N-m}=0$ (because $B$ is strictly upper triangular), a straightforward induction leads to

$$
K^{N-m}=\left(\begin{array}{c|c}
0 & C \\
\hline 0 & I_{m}
\end{array}\right), \quad \text { with } C=\sum_{k=0}^{N-m-1} B^{k} H,
$$

and $K^{n}=K^{N-m}$ for all $n \geq N-m$. If one denotes by $u^{0}, u^{1}, \ldots, u^{k}=K^{k} u^{0}, \ldots$, the successive opinion fields, the opinions of influencers propagates along the branches of the tree during the first iterates $1, \ldots$, up to $N-m$ at most, and it is then frozen to the fixed point for larger values of $k$.

Now consider the time continuous evolution problem (15). Given an initial condition $u^{0}$, the solution can be expressed as

$$
u^{t}=\exp \left(-\frac{t}{\eta} A\right) u^{0}=e^{-t / \eta} \sum_{k=0}^{+\infty}\left(\frac{t}{\eta}\right)^{k} \frac{K^{k}}{k !} u^{0}=\sum_{k=0}^{+\infty} a_{k}(t) u^{k}
$$

with

$$
a_{k}(t)=\frac{1}{k !} e^{-t / \eta}\left(\frac{t}{\eta}\right)^{k}
$$

The exact solution at time $t$ is then an infinite barycentric combination of the discrete iterates $\left(u^{k}\right)$, weighted by a Poisson distribution of parameter $t / \eta$. It makes it possible to distinguish two phases in the evolution: a transitional phase, shorter than $(N-m) \eta$ during which opinions evolves, and a second one, for $t$ larger than $(N-m) \eta$, where most of the mass of the Poisson distribution corresponds to discrete modes $u^{k}$ equal to the equilibrium state $u^{N-m}$, where the solution is close to this limit.

Remark 4.5. Note that the transitional phase can be significantly longer than $\eta$, which the characteristic time given by the stability analysis. This is a typical behavior of evolution equations associated to non-diagonalizable operators: flushing out the system of its initial state may take a time much longer than the characteristic damping time.

Remark 4.6. Hierarchical graph can take two types of extremal, tree-like, forms, depending on the sense of arrows (from the leafs to the root, or the other way around). The first one would be $m=1$, namely if there exists a unique influencer (see Figure 2 left). This corresponds to some kind of dictatorship since, at the end of the process (in no more than $N-1$ iterations for the discrete version of the model, where $N$ is the number of generations), the opinion of every agent will coincide with the one of the influencer. The other one would be the case when the root of the tree is forwardly connected to all the other vertices (see Figure 2, middle). This situation could be thought as a caricatural illustration of democracy, where the leafs are citizens (who are all influencers), and inner nodes correspond to some processes to gather and merge opinions in a hierarchical way. The opinion of the root is then a trade-off between the opinions of citizens. 

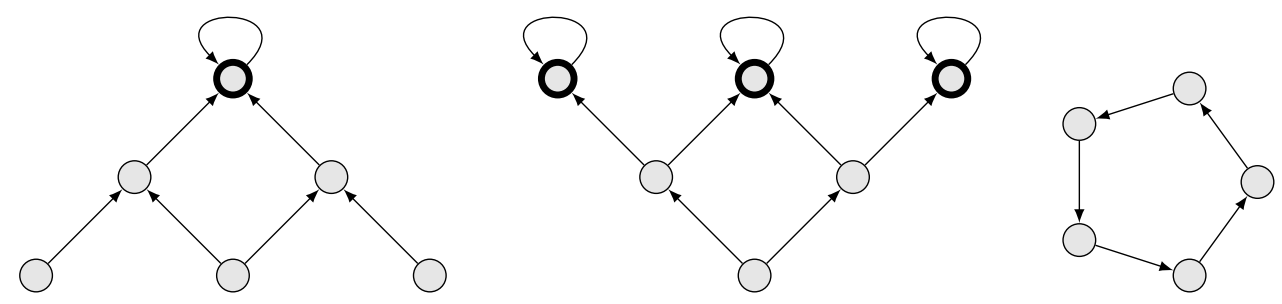

FiguRE 2. Three examples of networks which have not a gradient flow structure: a "dictatorial" hierarchical one, where there is only one influencer (left), a hierarchical "democratic" one, where the vertex in the bottom, thought as the leader, listens (indirectly) to everybody (middle), and a cyclical network of length 5 (right). Influencers are circled.

Pure cycle

The second situation corresponds to diagonalizable matrices with complex eigenvalues. An archetypal example is the case of a circulant matrix (see Figure 2, right, for the associated network), i.e. $K$ is a permutation matrix with a single cycle. We represent the set of vertices as $I_{N}=\{1, \ldots, N\}$, in such a way that the orbit of 1 is $1,2, \ldots, N, 1$, etc. . The matrix $K$ is a circulant matrix $\left(K_{1,2}=K_{2,3}=\cdots=K_{N, 1}=1\right)$, so that $K^{N}=I$ (identity matrix). Since the matrices $K^{n}$, for $n=0, \ldots, N-1$ are linearly independent, the minimal polynomial of $K$ is $X^{N}-1$. Its eigenvalues are therefore $\mu_{k}=\exp (2 i k \pi / N), k=0, \ldots, N-1$. This case appears as a limit case in terms of spectrum: the eigenvalues of any stochastic matrix are in the unit disc of $\mathbb{C}$ while, for this circulant matrix, they lie on the unit circle. To describe the time continuous solution, it is actually more convenient to use the native form of $K$, which expresses a leftward shift, rather than diagonalizing it. Indeed, for any initial vector $u^{0}$, the iterated vectors $u^{k}=K^{k} u^{0}$ are simply obtained by shifting the vector to the left, with periodicity, i.e. $u_{i}^{k+1}=u_{i+1}^{k}$. As mentioned previously for the hierarchical situation, the vector $u^{t}$ is simply the mean of the family $\left(u^{k}\right)_{k \in \mathbb{N}}$ with respect to the Poisson distribution of parameter $t / \eta$ :

$$
u^{t}=\exp \left(-\frac{t}{\eta} A\right) u^{0}=\sum_{k=0}^{+\infty} \frac{e^{-t / \eta}}{k !}\left(\frac{t}{\eta}\right)^{k} u^{k} .
$$

The evolution thus appears as a diffuse form of the backward transport phenomenon associated to the shift matrix. Note that it behaves exactly like the semi-discretized in space transport equation (see Section 6).

Note also that this situation is not so far from the linear network mentioned above, with two real eigenvalues, 1 and 0 , the latter being very degenerate. Indeed, simply disconnecting $N$ from itself, and rather connecting it to 1 , transforms this linear network onto a pure cycle.

Remark 4.7. (Additional comments on diagonalizability)

It may sound curious that being diagonalizable or not for a matrix may affect the behavior of the associated evolution model, because diagonalizability in $\mathbb{C}$ is generic for matrices (the set of diagonalizable matrices has full measure). One may be tempted to disregard this pathological character of not being diagonalizable, since it (almost) never happens in practice. Yet, we emphasize here that considering this case does actually make sense. Indeed, for some matrices which are close to the set of non-diagonalizable matrices (and thus far away from the set of normal matrices ${ }^{7}$ ), the behavior is better described by a formula of the type (17) rather than by a formula based on eigenvectors. Consider for example the case of a $N$-linear network detailed previously, with a single influencer, and a series of consecutive influenced agents. The corresponding matrix is "highly" non-diagonalizable, if one may say: 1 is an eigenvalue with multiplicity 1 , but 0 has multiplicity $N-1$, with a one-dimensional eigenspace. Now consider the case when the diagonal elements associated to $1,2, \ldots, N-1$, are replaced by $K_{11}, \ldots, K_{N-1, N-1}$, all positive values of order $\varepsilon$, and pairwise distinct. Since all the eigenvalues

\footnotetext{
${ }^{7} \mathrm{~A}$ matrix which commutes with its adjoint or, equivalently, a matrix which can be diagonalized in an orthogonal basis.
} 
are now distinct, the matrix is diagonalizable with eigenvalues $K_{11}, \ldots, K_{N-1, N-1}$ and 1 , and diagonalizing it makes it possible to express the solution as a linear combination of eigenvectors, with coefficients $e^{-t K_{11} / \eta}, \ldots$, $e^{-t \eta}$. In a modeling or numerical context, this approach must be avoided, because it does not allow a robust description of the behavior of the solution. Indeed, it can be checked that the eigenvectors associated to the $K_{i i}^{\prime} s$ are almost colinear, so that the change of basis matrix has a very high condition number (its smallest eigenvalue is close to 0), and the eigenvectors depend in a very stiff way upon the coefficients. To sum up, for such matrices which are diagonalizable in theory, but very close to non-diagonalizable matrices, the expected behavior shall be better described by an expression of the type (17), rather than on the expression based on eigenvectors. To put it another way, it seems reasonable to consider that diagonalizing the matrix in order to express the exact solution of the evolution problem should be restricted to matrices with a controlled non-normality (see e.g. [36]).

\section{Charismatic networks}

As mentioned above, we are interested in asymmetric networks: the influence that $x$ exerts on $y$ may be different from the one that $y$ exerts on $x$. However, by the introduction individual weights, one can recover some sort of symmetry in the interactions. We shall call these networks charismatic given the situation we aim at modeling, though a more standard name could be resistive networks or reversible Markov chains.

Definition 5.1. We say that the network $V$ with the matrix $\left(K_{x y}\right)_{x, y \in V}$ describing mutual influence if charismatic if there exists a strictly positive scalar field $\omega: V \rightarrow(0,+\infty)$ such that for all $x, y \in V$,

$$
\omega_{x} K_{x y}=\omega_{y} K_{y x} .
$$

Any strictly positive field $\omega: V \rightarrow(0,+\infty)$ such that 18$)$ holds for all $x, y \in V$ will be called a charisma of the network.

Remark 5.2. If we view $K$ as a Markov kernel on the set $V$, which means that $K_{x y}$ is the probability to jump from $x$ to $y$, then Definition 5.1 corresponds to a reversible Markov chains, and $\omega$ would be an invariant measure.

Remark 5.3. If a network is charismatic, then, $K_{x y}>0$ if and only if $K_{y x}>0$. As a consequence, if there exists a path from $x$ to $y$, then there also exists a path from $y$ to $x$. In particular, any influencer $x \in \Gamma$ is isolated, hence influencers will not have any effect upon the dynamic. Given the situation we aim at modeling, we will be interested only in strongly connected charismatic network: it excludes the presence of influencers.

Remark 5.4. Equation (18) can be written

$$
K_{x y}=\frac{\omega_{y}}{\omega_{x}} K_{y x}
$$

In other words, the influence that $y$ exerts on $x$ depends on the ratio of the charisma of $x$ and $y$, and the influence that $x$ exerts on $y$. The higher the charisma of $y$ is compared to the one of $x$, the more $y$ influences $x$ compared to the reciprocal influence of $x$ onto $y$. It justifies the name charisma: the higher the charisma of an agent is, the more they influence the others while not being influenced by them.

In particular, the vertex $x$ would have a behavior close to the one of an influencer if $\omega_{x}$ is very large compared to the other values taken by the charisma.

Remark 5.5. If a network is charismatic and if $\bar{\omega}: V \rightarrow(0,+\infty)$ is a charisma, then so is $\lambda \bar{\omega}$ for any $\lambda>0$. On the other hand, provided that the network is strongly connected, any charisma $\omega$ is of the form $\lambda \bar{\omega}$ for some $\lambda>0$. Indeed, let $\omega$ be another charisma. We define $\lambda=\omega_{x} / \bar{\omega}_{x}$ for some arbitrary $x \in V$. If $y$ is any neighbor of $x$,

$$
\omega_{y}=\omega_{x} \frac{K_{x y}}{K_{y x}}=\omega_{x} \frac{\bar{\omega}_{y}}{\bar{\omega}_{x}}=\lambda \bar{\omega}_{y} .
$$


Propagating this relation from one vertex to its neighbors, and using the strong connectivity of the graph, we conclude that $\omega=\lambda \bar{\omega}$ on $V$.

The next proposition gives a description of the set of matrices associated to charismatic networks.

Proposition 5.6. Let $\mathcal{S} \subset \mathbb{R}^{V \times V}$ be the set of symmetric matrices $C$ with nonnegative entries and such that $(V, \operatorname{supp}(C))$ is a strongly connected graph, and let $\sim$ the equivalence relation defined by $C_{1} \sim C_{2}$ if $C_{1}=\lambda C_{2}$ for some $\lambda>0$.

There exists a homeomorphism between $\mathcal{S} / \sim$ and the set of matrices $K \in \mathbb{R}^{V \times V}$ for which $(V, K)$ is a strongly connected charismatic network.

As a consequence, we can think at the set of matrices $K \in \mathbb{R}^{V \times V}$ for which $(V, K)$ is a charismatic network as a $(N(N+1) / 2-1)$-dimensional manifold, where $N$ is the number of vertices of $V$.

Proof. If $K$ describes a charismatic network with charisma $\omega$, we map it onto $F(K)$ the equivalence class of $C$ defined by $C_{x y}=\omega_{x} K_{x y}$. Indeed, this matrix belongs to $\mathcal{S}$ by (18), and its equivalence class for the relation $\sim$ does not depend on the choice of charisma by Remark 5.5 .

Conversely, if $C \in \mathcal{S}$, we can define

$$
\omega_{x}=\sum_{y} C_{x y} .
$$

and then we map $[C]$ onto $K=G([C])$ defined by $K_{x y}=C_{x y} / \omega_{x}$. The network described by $K$ is charismatic, the map is well-defined in the sense $K$ does not depend on the choice of a representative in the equivalence class of $C$ for $\sim$ : indeed, multiplying $C$ by $\lambda>0$ would also multiply $\omega$ by $\lambda$, hence $K$ would be the same.

Moreover, both $F$ and $G$ are continuous, and it can be checked easily that $F=G^{-1}$.

Now, let us describe more in details the structure of charismatic networks. We wrote above in Remark 1.5 that the total opinion is in general not conservative. However, in the case of charismatic networks, a certain quantity is conserved, that is the expected value of the opinion field with respect to the measure $\left(\omega_{x}\right)$. At time $k$, this quantity writes

$$
v^{k}=\sum_{x \in V} \omega_{x} u_{x}^{k}
$$

It does not depend on $k$ provided $\omega$ is an admissible charisma. Indeed, it holds that

$$
v^{k+1}=\sum_{x \in V} \omega_{x} u_{x}^{k+1}=\sum_{x, y \in V} \omega_{x} K_{x y} u_{y}^{k}=\sum_{x, y \in V} \omega_{y} K_{y x} u_{y}^{k}=\sum_{y \in V} \omega_{y} u_{y}^{k} \sum_{x \in V} K_{y x}=\sum_{y \in V} \omega_{y} u_{y}^{k}=v^{k} .
$$

It makes it possible to characterize the uniform field to which the distribution of opinions converges.

Proposition 5.7. Assume that the network is charismatic and that the assumption of Corollary 1.8 holds. Let $\left(\omega_{x}\right)_{x \in V}$ the charisma satisfying the normalization condition $\sum_{x \in V} \omega_{x}=1$ (given Remark 5.5 there is only one such charisma). Then the solution $u^{k}$ of the evolution (2) converges to a uniform field defined by

$$
u^{\infty}=\sum_{x \in V} \omega_{x} u_{x}^{0}
$$

In short: the final value of the distribution of opinions is a convex combination of the initial values of the opinion, and the coefficients in the combination are nothing else than the charisma of the agents. In particular, the higher the charisma of an agent $x$, the higher the influence of $u_{x}^{0}$ in the value reached by the opinion at consensus.

Proof. By Corollary 1.8 , we know that the distribution of opinions converges to a uniform field. Using the fact that $v^{k}$ does not depend on $k$, and calling $u^{\infty}$ the final value, we obtain

$$
\sum_{x \in V} \omega_{x} u^{\infty}=\lim _{k \rightarrow+\infty} \sum_{x \in V} \omega_{x} u_{x}^{k}=\sum_{x \in V} \omega_{x} u_{x}^{0}
$$


Using the normalization of the charisma, we deduce the result.

Variational standpoint, gradient flow structure $\&$ resistive networks

Another key feature, in a charismatic network, is the relevance of a variational point of view. The propagation can be described with the help of an energy functional defined on the set of configurations. Assume that the network is charismatic with charisma $\omega$. We define $\Phi: \mathbb{R}^{V} \rightarrow[0,+\infty)$ in the following way: for any $u \in \mathbb{R}^{V}$,

$$
\Phi(u)=\sum_{e \in E} \frac{\omega_{x} K_{x y}}{2}\left(u_{x}-u_{y}\right)^{2}
$$

In the spirit of Section 4 , the continuous time problem for a charismatic network $K$ is the gradient flow of the energy $\Phi$ with respect to the scalar product defined by

$$
\langle u \mid v\rangle_{\omega}=\sum_{x \in V} \omega_{x} u_{x} v_{x}
$$

As a consequence, the energy $\Phi$ decreases along the continuous time evolution, and converges (under the assumption that the network is connected) to a minimizer of $\Phi$, namely a uniform field.

With the help of this energy, we can make the link with resistive networks. The analogy would be to think at $u_{x}$ as the potential at the vertex $x$, while the quantity $\omega_{x} K_{x y}$ (which is symmetric in $x, y$ ) would be the conductance (the inverse of a resistance) of the edge joining $x$ to $y$. Then, $\Phi(u)$ would be nothing else than (twice) the energy dissipated in the electrical circuit with conductances $\omega_{x} K_{x y}$ and electrical potentials $u_{x}$.

\section{Extension to infinite networks}

The variational structure of the problem makes it possible to explore the case of infinite networks with standard tools in functional analysis. In particular, consider a connected resistive network $(V, E, C, o)$ that is rooted (an arbitrary vertex $o$ has been singled out, and is considered to exchange flux with the outside world), infinite, non oriented, i.e. $E \subset V \times V$ is symmetric, and $C \in(0,+\infty)^{E}$ is a symmetric collection of conductances (inverse of resistances). Let us first say that the collection of conductances canonically defines a metric on the graph: the length of a path is simply the sum of conductances of the edges, and the distances between two vertices is the infimum of the lengths of paths joining them. Assuming that the number of neighbors for each vertex is finite, one may define a natural "energy space", discrete version of a standard Sobolev space for functions defined over a Euclidean domain, as

$$
H^{1}=\left\{u \in \mathbb{R}^{V}, u_{0}=0, \sum_{(x, y) \in E} C_{x y}\left(u_{y}-u_{x}\right)^{2}<+\infty\right\} .
$$

Here $u$ can be interpreted as a collection of pressures at vertices (if one considers that a fluid flows within edges), electric potentials, or opinion if one sticks to our main topic. In a fluid or electric context, the quantity involved in the definition of $H^{1}$ is the dissipated energy 8 , which is twice the quantity previously defined as $\Phi(u)$ (see (20)). The core questions is now the following: are there nontrivial harmonic fields, i.e. solutions to

$$
\sum_{x \rightarrow y} C_{x y}\left(u_{x}-u_{y}\right)=0 \quad \forall x \in \stackrel{\circ}{V}=V \backslash\{o\}
$$

which belong to the Hilbert space $H^{1}$ ? Such nontrivial solutions correspond, in the fluid dynamic context, to the case when some fluid enters and exits the domain through infinity, with a finite amount of dissipated energy. In the case of opinion networks, it would correspond to influencers "located at infinity", who yet exert some 
influence on standard agents. Note that, due to the infinite character of the network, those influencers are not represented by vertices, they should rather be seen as particular paths toward infinity. Notice that, in many situations (for example with binary trees), this set of ends is uncountable, while the set of vertices is. Let us define $R_{n}$ has the equivalent resistance between $o$ and the vertices at distance $n$ (in terms of number of edges) from $o$. This quantity increases, thus converges to some limit value $R \in(0,+\infty]$. It can be proved (see [59]) that, as soon a $R$ is finite, there exist nontrivial solutions to the problem above. In other words, if one defines $H_{0}^{1}$ as the closure of the fields which have a finite number of nonzero entries, the quotient space $H^{1} / H_{0}^{1}$ is not trivial. It makes it possible to properly define a Dirichlet problem, where the boundary condition (that would be the collection of influencers' opinions) is set (in some abstract way) at infinity. We refer the reader to [14 45] for an application of this approach to a fully different problem, that is the modeling of the human respiratory system. To make a connection with Section 2, more precisely with Proposition 2.1. let us add that the finite character of the resistance $R$ is equivalent to the recurrent character of the random walk canonically associated to the network, i.e. with transition probabilities

$$
K_{x y}=C_{x y} / C_{x}, C_{x}=\sum_{x \rightarrow y} C_{x y}
$$

where the normalization constant $C_{x}$ is actually the charisma $\omega_{x}$ introduced at the beginning of this section. We refer to 28,59 for more details on these issues.

Remark 5.8. (Hierarchical / reversible networks \& Wasserstein-like gradient flow structure) In the situation considered in this section, i.e. if $K$ is a charismatic network with charisma $\omega: V \rightarrow(0,+\infty)$, we just noticed that the evolution equation

$$
\frac{d u}{d t}=-(I-K) u
$$

has a gradient flow structure subject to an appropriate choice of the Euclidean structure, for the quadratic functional given above. Recent advances in Optimal Transportation on networks have exhibited an other underlying gradient flow structure, in a fully different sense. Let us first notice that, like in the discrete setting, the quantity $\sum \omega_{x} u_{k}$ is preserved by the time-continuous equation, so $\omega_{x} u_{x}$ can be seen (up to an adapted normalization of the initial condition), as a probability measure on $V$. The approach, introduced in [43], is based on defining a metric on this very space of probability measures on $V$. This metric mimics the standard Wasserstein distance defined for measures on a Euclidean domain (see e.g. [57]), it relies on an extension to the discrete setting to the Benamou-Brenier approach (see [11]). It can be shown that the evolution equation is indeed a gradient flow, in this framework, for a functional that is defined as the relative entropy of the probability measure $\left(\omega_{x} u_{x}\right)$ with respect to the stationary measure / charisma $\left(\omega_{x}\right)$, i.e.

$$
\mathcal{H}(u)=\sum_{x} \omega_{x} u_{x} \log \left(u_{x}\right)
$$

We also refer the reader to 5 for a link between this framework and the space-discretization of Partial Differential Equations of the transport-diffusion type (like in Section 6) with a transport velocity which is the gradient of some scalar field.

\section{Space time discretization schemes for Partial Differential Equations}

We describe the discrete dynamical systems which come from the space and time discretization of standard Partial Differential Equations. This section is independent of the rest, it aims at underlying the fact that

${ }^{8}$ We must admit that the $H^{1}$ seminorm has no clear meaning in the context of opinion propagation. 

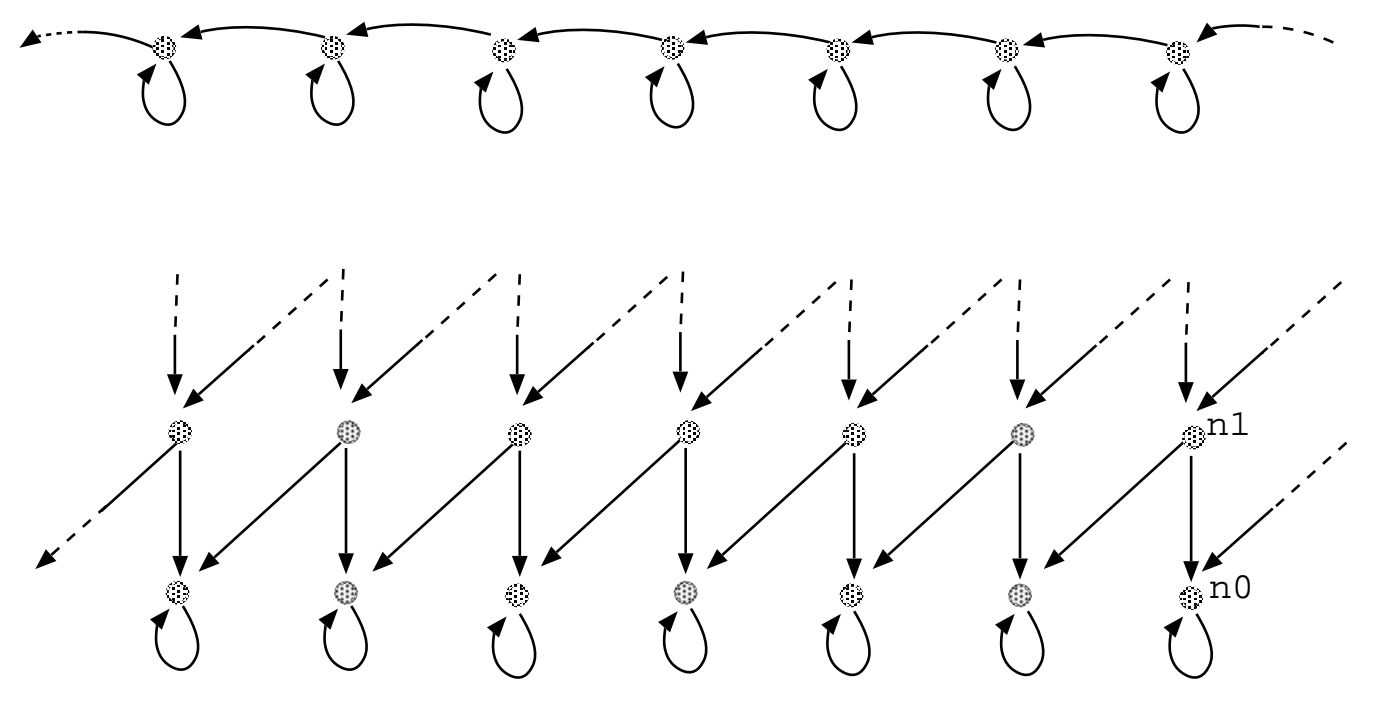

FiguRE 3. Upwind scheme graphs: space graph (top) and full space-time graph (bottom).

evolution problems of the type (2) can be seen as discrete generalization of standard PDE's. In the onedimensional setting, we shall consider the transport equation at constant velocity $U>0$

$$
\partial_{t} u+U \partial_{x} u=0
$$

and the heat equation

$$
\partial_{t} u-D \partial_{x x} u=0
$$

where $D>0$ is the diffusion coefficient. The space-time variable $(x, t)$ belongs to $[0, L] \times[0, T]$. We shall assume periodic boundary conditions in space, i.e. $L$ is identified to 0 . The Finite Difference space-time discretization of such equations is based on a time step $\Delta t=T / N_{T}$ and space step $\Delta x=L / N$. We shall denote by $u_{j}^{k}$ the approximation of a solution to one of those equations at $(j \Delta x, k \Delta t)$. Given an initial condition $u^{0}$, we build an initial discrete approximation according to $u_{j}^{0}=u^{0}(j \Delta x)$. The so-call upwind scheme for 21) writes

$$
\frac{u_{j}^{k+1}-u_{j}^{k}}{\Delta t}+U \frac{u_{j}^{k}-u_{j-1}^{k}}{\Delta x}=0 \quad j=1, \ldots, N \equiv 0, k=0, \ldots, N_{T}-1 .
$$

The previous scheme can be written

$$
u_{j}^{k+1}=\underbrace{\left(1-\frac{U \Delta t}{\Delta x}\right)}_{=K_{j j}} u_{j}^{k}+\underbrace{\frac{U \Delta t}{\Delta x}}_{=K_{j, j-1}} u_{j-1}^{k},
$$

which fits into the framework described at the beginning of Section 1 (Equation 2), as soon as the so -called CFL condition $\theta=U \Delta t / \Delta x \leq 1$ is satisfied. Vertices identify here with space discretization points, and the underlying graph is represented in Figure 3 (top). Note that for $\theta=1$, we recover the one-to-one and onto mapping situation. For $\theta \in(0,1)$, the problems fits in the assumptions of Corollary 1.8 (for $m=N$ ), thus $u^{k}$ converges to a uniform field. This results may appear as conflicting with pure transport phenomena, which do not change the profile. It is a consequence of the so-called numerical diffusion, which is a consequence of space discretization. Note that the set of harmonic fields is the line spanned by the uniform field $(1, \ldots, 1)$. 
For the heat equation, the so-called explicit scheme writes

$$
\frac{u_{j}^{k+1}-u_{j}^{k}}{\Delta t}+D \frac{-u_{j-1}^{k}+2 u_{j}^{k}-u_{j+1}^{k}}{(\Delta x)^{2}}=0 \quad j=1, \ldots, N \equiv 0, k=0, \ldots, N_{T}-1
$$

which can be written

$$
u_{j}^{k+1}=\underbrace{\left(1-\frac{2 D \Delta t}{(\Delta x)^{2}}\right)}_{=K_{j j}} u_{j}^{k}+\underbrace{\frac{D \Delta t}{(\Delta x)^{2}}}_{=K_{j, j-1}} u_{j-1}^{k}+\underbrace{\frac{D \Delta t}{(\Delta x)^{2}}}_{=K_{j, j+1}} u_{j+1}^{k} .
$$

Under the stability condition $2 D \Delta t / D x p^{2} \leq 1$, this expresses again an evolution of the type (2). Note that the network associated to the heat equation (discretization scheme (24)) is charismatic (according to Definition 5.1), whereas the transport network (associated to the discretization scheme (23)) is not.

\section{Space-time graph}

Now consider the space-time discrete field $\left(u_{j}^{n}\right)$ obtained by one of the previous schemes, for the transport equation or the heat equation. We replace the index $k$ by $n$ because the time index no longer plays the role of an incremental evolution index. It can be seen as a discrete field over the space-time grid

$$
V=\left\{(k, j), 0=1, \ldots, N, k=0, \ldots, N_{T}\right\}
$$

In this setting, the topology of the graph is represented in Figure 3 (bottom), and the vertices corresponding to the initial states, at which the values are prescribed, play the role of influencers. The full discrete field can thus be considered as harmonic in the sense of 1.2 , over the space-time grid. For a given grid, there is obviously a unique harmonic field in this sense for any collection of initial values (i.e. values at influencers), so harmonic fields form a linear space of dimension $N$.

\section{Nonlinear EVOlution models}

In this section we investigate ways to model the opinion propagation between two agent in a more realistic way. In the previous section, the "flux" of opinion was considered linear, which means in particular that a person with an opinion far away from that of an agent is likely to exert a strong influence on this agent. It seems more relevant to consider that the actual influence decreases when the opinion diverges. In order to incorporate this effect, and possibly others, to the model, we consider now that the flux of opinion (see Remark 1.3 is no longer linear. We denote this opinion flux from $y$ to $x$ by $K_{x y} \varphi(v-u)$, which leads to the following evolution model (in the discrete setting):

$$
u_{x}^{k+1}=u_{x}^{k}+\sum_{x \rightarrow y} K_{x y} \varphi\left(u_{y}^{k}-u_{x}^{k}\right) .
$$

To encode the bounded confidence effect mentioned previously, one may prescribe that $\varphi(u)$ is small for large values of $u$. Examples of functions $\varphi$ that we will consider are displayed in Figure 4.

For an arbitrary function $\varphi$, conducting a full analysis of the system goes far beyond the scope of these notes, and may be simply out of reach in full generality. We shall restrict ourselves to a particular class of nonlinearities:

Definition 7.1. (Admissible non linearities)

We say that $\varphi: \mathbb{R} \rightarrow \mathbb{R}$ is admissible if it is continuous, odd, and if $0 \leq \varphi(u) \leq u$ for all $u \geq 0$.

The first assumption simply expresses stability with respect to opinions, and the odd-character corresponds to the fact that agents are interchangeable as far as opinion propagation is concerned. Nonnegativity of $\varphi$ rules out any contradiction spirit: we restrict ourselves to model consensual tendencies. The last condition $\varphi(u) \leq u$ 


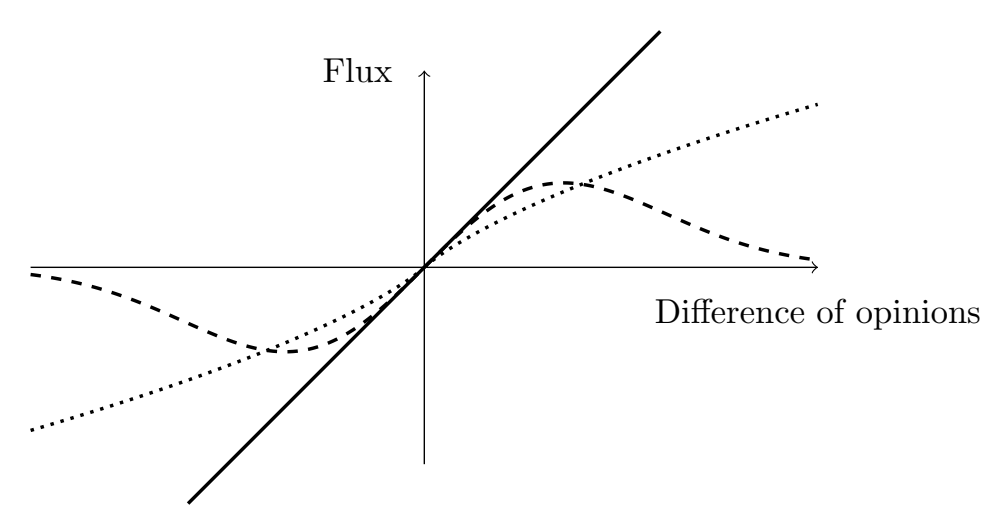

Figure 4. Examples of admissible $\varphi$ for the non linear model. The solid line corresponds to the linear model. The dotted line is an example of admissible $\varphi$ which satisfies $\varphi^{\prime} \in(0,1]$. The dashed line is an admissible $\varphi$ with a bounded confidence effect, i.e. agents are not affected by people with a very different opinion from theirs.

ensures that, if an agent $x$ which thinks $u$ is influenced by an agent $y$ which thinks $v$, with $u \leq v$, then the opinion of agent $x$ will be above $u$ but below $v$, and not beyond $v$. The two latter conditions will ensure that the maximum principle is preserved.

Remark 7.2. We require $\varphi$ to be defined on the whole $\mathbb{R}$ but this is more a mathematical convenience than a relevant modeling requirement. Indeed, as we will see later in Proposition 7.4, the maximum principle stays true. As a consequence, it would be enough to define $\varphi$ on $[-M, M]$, where $M=\max u^{0}-\min u^{0}$, where $\left(u_{x}^{0}\right)_{x \in V}$ is the initial distribution of the opinions. Nevertheless, not to overburden notations, we will keep the assumption that $\varphi$ is defined on $\mathbb{R}$.

Similarly to the linear case, a fixed point is a situation where nobody changes their opinion.

Definition 7.3. (Nonlinear fixed point)

We say that $u \in \mathbb{R}^{V}$ is a fixed point for 25 whenever

$$
u_{x}=u_{x}+\sum_{x \rightarrow y} K_{x y} \varphi\left(u_{y}-u_{x}\right)
$$

To prove the existence of at least one fixed point, we need first to state the maximum principle. Thanks to the assumptions on $\varphi$, it stays valid

Proposition 7.4. (Nonlinear maximum principle)

Let $\varphi$ be admissible in the sense of Definition 7.1. Let an initial state $u^{0} \in \mathbb{R}^{V}$ be given, and let $u^{1}, \ldots, u^{k}$, ... the fields recursively defined according to 25). Then, for any $k \geq 0$, for any $x \in V$,

$$
u_{x}^{k+1} \in\left[\min u^{k}, \max u^{k}\right] \subset\left[\min u^{0}, \max u^{0}\right]
$$

Proof. Fix some $k \geq 0$ and $x \in V$. Among the $y$ such that $x \rightarrow y$, there are some such that $u_{y}^{k} \geq u_{x}^{k}$ and the ones for which $u_{y}^{k}<u_{x}^{k}$. The second ones yield a negative flux because $\varphi$ is odd and takes nonnegative values 
on $\mathbb{R}_{+}$. Thus we can write, using $\varphi(u) \leq u$ for $u \geq 0$,

$$
\begin{aligned}
u_{x}^{k+1} & =u_{x}^{k}+\sum K_{x y} \varphi\left(u_{y}^{k}-u_{x}^{k}\right) \leq u_{x}^{k}+\sum_{u_{y}^{k} \geq u_{x}^{k}} K_{x y} \varphi\left(u_{y}^{k}-u_{x}^{k}\right) \\
& \leq u_{x}^{k}+\sum_{u_{y}^{k} \geq u_{x}^{k}} K_{x y}\left(u_{y}^{k}-u_{x}^{k}\right) \leq u_{x}^{k}+\underbrace{\left(\sum_{u_{y}^{k} \geq u_{x}^{k}} K_{x y}\right)}_{\leq 1}\left(\max u^{k}-u_{x}^{k}\right) \leq \max u_{k} .
\end{aligned}
$$

As $\varphi$ is odd, $-u^{k}$ also satisfies 25 thus by the same reasoning $u_{x}^{k+1} \geq \min u^{k}$ for all $k \geq 0$ and $x \in V$. The property follows by induction on $k$.

The existence of at least at fixed point, in this non linear setting, is still guaranteed by Brouwer's Theorem.

Proposition 7.5. Assume that $\varphi$ is admissible in the sense of Definition 7.1. Let $U \in \mathbb{R}^{\Gamma}$ be a given data on $\Gamma$. Then there exists $u \in \mathbb{R}^{V}$ a fixed point, i.e. a solution of (26) such that $u=U$ on $\Gamma$.

Proof. We denote by $a$ and $b$ respectively the minimum and maximum of $U$ over $\Gamma$. Recall that $\stackrel{\circ}{V}=V \backslash \Gamma$. We denote $\Lambda_{a}^{b}=[a, b]^{V}$ and we define $F: \Lambda_{a}^{b} \rightarrow \Lambda_{a}^{b}$ the map defined by

$$
[F(u)]_{x}=u_{x}+\sum_{x \rightarrow y} K_{x y} \varphi\left(u_{y}-u_{x}\right)
$$

for all $x \in V_{0}$. Thanks to Proposition 7.4, we know that this map, defined over $\Lambda_{a}^{b}$ takes indeed its values in $\Lambda_{a}^{b}$. The map $F$ being continuous and as $\Lambda_{a}^{b}$ is convex and compact, Brouwer's Theorem (Theorem 6.6 in [17]) asserts that $F$ admits a fixed point $v \in \Lambda_{a}^{b}$. Then, the fixed point $u$ we are looking for is defined as $u=U$ on $\Gamma$ and $u=v$ on $\stackrel{\circ}{V}$.

The natural questions are the ones of uniqueness and stability of the fixed point. As examples below shall show, this is not true in general. However, with a restrictive assumption on $\varphi$ we can show that everything happens like in the linear case.

Proposition 7.6. Take an admissible $\varphi$ in the sense of Definition 7.1. We assume moreover that $\varphi$ is differentiable and $\varphi^{\prime}(u) \in(0,1]$ for all $u \in \mathbb{R}$.

Similarly to Proposition 1.10, assume that $\Gamma \neq \emptyset$ and that $V-\cdot \rightarrow \Gamma$. Then, for any given data $U$ on $\Gamma$, the problem admits a unique fixed point, and, for any initial data $u^{0}$ with $u^{0}=U$ on $\Gamma$, the evolution problem (25) converges to this fixed point as $k \rightarrow+\infty$.

Proof. Let $u$ and $v$ be two solutions of the evolution process. Using the mean value theorem, there exist numbers $\left(\alpha_{x y}^{k}\right)$ in $(0,1]$ such that for all $k \geq 0$ and any $x \in V$,

$$
u_{x}^{k+1}-v_{x}^{k+1}=u_{x}^{k}-v_{x}^{k}+\sum_{x \rightarrow y} K_{x y} \alpha_{x y}^{k}\left(u_{y}^{k}-v_{y}^{k}+v_{x}^{k}-u_{x}^{k}\right)
$$

Indeed, we know that $\alpha_{x y}^{k}=\varphi^{\prime}(z)$ for some $z$ which depends on $u_{x}^{k}, v_{x}^{k}, u_{y}^{k}$ and $v_{y}^{k}$. By boundedness of the families $\left(u_{x}^{k}\right)_{k, x},\left(v_{x}^{k}\right)_{k, x}$ (which derives from the maximum principle), we know that $\underline{\alpha} \leq \alpha_{x y}^{k} \leq 1$ where $\underline{\alpha}>0$ is independent on $k, x$ and $y$. Denoting $w^{k}=u^{k}-v^{k}$, we know that $w^{k}=0$ on $\Gamma$ and that $w^{k}$ satisfies a linear evolution equation (with coefficients depending on $k$ )

$$
w_{x}^{k+1}=w_{x}^{k}+\sum_{x \rightarrow y} K_{x y} \alpha_{x y}^{k}\left(w_{y}^{k}-w_{x}^{k}\right) .
$$


As already underlined in the proof of Proposition 1.11 we just need to show that $w^{k}$ tends to 0 as $k \rightarrow+\infty$ to prove our claim.

Let us denote by $\hat{K}^{k}$ the matrix defined by

$$
\hat{K}_{x y}^{k}= \begin{cases}\alpha_{x y}^{k} K_{x y} & \text { if } x \neq y \\ 1-\sum_{z} \alpha_{x z}^{k} K_{x z} & \text { if } x=y\end{cases}
$$

With the help of this notation, the equation satisfies by $w$ can be written $w^{k+1}=\hat{K}^{k} w^{k}$, where the product is understood as a matrix-vector product. As $0 \leq \alpha_{x y}^{k} \leq 1$, the coefficients of $\hat{K}$ are nonnegative. Moreover, the sum of each row of $\hat{K}^{k}$ is 1 . In particular, the sum of each row of the matrix $\hat{K}^{k} \hat{K}^{k-1} \cdots \hat{K}^{1} \hat{K}^{0}$ is also 1. In consequence, for each $x \in V$ and each $k \geq 0, w_{x}^{k}$ is a weighted barycenters of the $\left(w_{y}^{0}\right)_{y \in V}$ with weights depending on $k$ and $x$.

Now let us fix $x \in V$. Recall that $E \subset V \times V$ is the set of directed edges of the network. By assumption, there exists an injective path $\left(x=x_{\ell}, x_{\ell-1}, \ldots, x_{1}, x_{0}=y\right)$ connecting $x$ to $y \in \Gamma$ with $\left(x_{i+1}, x_{i}\right) \in E$ for all $i$. As a consequence, the coefficient $x y$ in the matrix $\hat{K}^{\ell-1} \hat{K}^{\ell-2} \cdots \hat{K}^{1} \hat{K}^{0}$ is larger than

$$
\left(\alpha_{x_{\ell} x_{\ell-1}}^{\ell-1} K_{x_{\ell} x_{\ell-1}}\right)\left(\alpha_{x_{\ell-1} x_{\ell-2}}^{\ell-2} K_{x_{\ell-1} x_{\ell-2}}\right) \cdots\left(\alpha_{x_{1} x_{0}}^{0} K_{x_{1} x_{0}}\right) \geq(\underline{\alpha})^{l}\left(\min _{\left(z_{1}, z_{2}\right) \in E} K_{z_{1} z_{2}}\right)^{\ell}=c^{\ell}
$$

where we have set $c=\underline{\alpha}\left(\min _{\left(z_{1}, z_{2}\right) \in E} K_{z_{1} z_{2}}\right)>0$. In other words, this coefficient is bounded from below by a constant $c^{\ell} \in(0,1]$ that depends only on $\underline{\alpha}, K$ and $\ell$. With the help of this estimate, given the worst case situation,

$$
w_{x}^{\ell} \leq\left(1-c^{\ell}\right)\left(\max _{z} w_{z}^{0}\right)+c^{\ell} w_{y}^{0}=\left(1-c^{\ell}\right)\left(\max _{z} w_{z}^{0}\right),
$$

as we have used that $y \in \Gamma$ so $w_{y}^{0}=0$. Notice that this estimate can be iterated. As the constant $c^{\ell}$ does not depend on $k$, it leads to

$$
w_{x}^{n l} \leq\left(1-c^{\ell}\right)^{n}\left(\max _{z} w_{z}^{0}\right),
$$

for all $n \geq 1$. Now take $k$ of the form $n L$ where $L$ is the least common multiple of all the lengths of paths connecting elements of $V$ to $\Gamma$. We can write

$$
\max _{x \in V} w_{x}^{n L} \leq\left(1-c^{L}\right)^{n}\left(\max _{x} w_{x}^{0}\right) .
$$

As a similar inequality holds for $-w^{n L}$, we conclude that $w^{n L}$ tends to 0 as $n \rightarrow+\infty$. By the maximum principle, it in fact implies that $w^{k}$ tends to 0 as $k \rightarrow+\infty$, which concludes the proof.

However, in the case when $\varphi^{\prime} \in(0,1]$ does not hold, we know that we cannot expect uniqueness nor stability of fixed points, this is object of the two following remarks. Notice that if $\varphi(u)$ tends to 0 as $u$ grows, which corresponds to a bounded confidence effect, we necessarily have $\varphi^{\prime}(u)<0$ for some $u$.

Remark 7.7. (Non uniqueness)

If $\varphi$ is not injective, then uniqueness cannot hold. Indeed, if $\varphi(\bar{u})=\varphi(\bar{v})$ for $\bar{u} \neq \bar{v}$ then it is enough to take this simple model: $V=\{0,1,2\}$ and 0 and 2 are influencers, see Figure 5 . Moreover, 1 listens equally to 0 and 2 , and not to itself. We put $u_{0}=\bar{u}$ and $u_{2}=-\bar{v}$. Then one easily check that both $u_{1}=0$ and $u_{1}=\bar{u}-\bar{v}$ are fixed points. Moreover, as $\varphi$ is odd, taking $u_{1}=(\bar{u}-\bar{v}) / 2$ yields a third fixed point.

Remark 7.8. (Instabilities)

Stability analysis is related to the values taken by $\varphi^{\prime}$. Indeed, if $u \in \mathbb{R}^{V}$ is a fixed point, the linearized system 


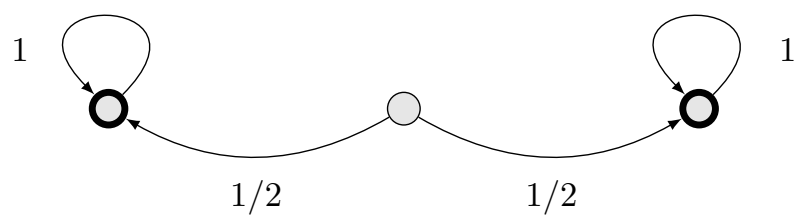

Figure 5. The very simple network with 3 vertices and 2 influencers (circled nodes) which helps to understand what may happen if the assumption $\varphi^{\prime} \in(0,1]$ does not hold.

around $u$, for a perturbation of the form $u+\varepsilon v$ reads, at the first order in $\varepsilon$,

$$
v_{x}^{k+1}=v_{x}^{k}+\sum_{x \rightarrow y} K_{x y} \varphi^{\prime}\left(u_{y}-u_{x}\right)\left(v_{y}^{k}-v_{x}^{k}\right) .
$$

Now, if $\varphi^{\prime}(u) \in[0,1]$, the linearized system can be written $v^{k+1}=\tilde{K} v^{k}$ where $\tilde{K}_{x y}=K_{x y} \varphi^{\prime}\left(u_{y}-u_{x}\right)$ defines a matrix with nonnegative entries such that the sum of the rows is smaller than 1 . As a consequence, we can use the maximum principle on the linearized system and conclude that it is stable. Actually, we already knew it thanks to Proposition 7.6. On the other hand, let $\bar{u}>0$ be some value of opinion. Consider again the very simple graph $V=\{0,1,2\}$ where 0 and 2 are influencers and 1 listens equally to both influencers, see Figure 5 . If $u_{0}=\bar{u}$ and $u_{2}=-\bar{u}$ then $u_{1}=0$ is a fixed point. The linearized system around this fixed point reads

$$
v_{1}^{k+1}=v_{1}^{k}-\frac{\varphi^{\prime}(\bar{u})}{2} v_{1}^{k}-\frac{\varphi^{\prime}(-\bar{u})}{2} v_{1}^{k}=\left(1-\varphi^{\prime}(\bar{u})\right) v_{1}^{k},
$$

where we use that $\varphi^{\prime}$ is even as $\varphi$ is odd. In particular, if $\varphi^{\prime}(\bar{u})<0$ or $\varphi^{\prime}(\bar{u})>2$, we see immediately that this fixed point is unstable.

\section{INERTIAL EVOLUtion MODEL, INSTABILITIES}

We previously introduced and analyzed in Section 4 the first order in time model

$$
\frac{d u}{d t}=-\frac{1}{\eta}(I-K) u=-\frac{1}{\eta} A u
$$

where $K$ is a stochastic matrix. The vector $u \in \mathbb{R}^{V}$ represents opinions of agents indexed by vertices in a set $V$, and $u_{\mid t=0}=u^{0} \in \mathbb{R}^{V}$ is prescribed as an initial opinion. As detailed in the previous sections, it models the evolution of an opinion field $u$ on a network $(V, E)$, where $E=\operatorname{supp}\left(K_{x y}\right)$, and $K_{x y}$ accounts for the influence of $y$ upon $x$.

We shall consider here an inertial version of this model. Let us make it clear that this model is more an abstract development than a real attempt to better describe the reality of opinion propagation. Let us nevertheless give a few words on the underlying principles, as questionable as they may be: we aim at implementing the idea than someone may react to an influence (caused by the discrepancy between their own opinion and an another agent's opinion) with some "psychological inertia": an agent $x$ is submitted to the influence of $y$, this influence can be formalized by a flux (see Remark 1.3 equal to $K_{x y}\left(u_{y}^{k}-u_{y}^{k}\right)$, and we shall consider that this flux pushes in some way $x$ 's mind, possibly further in the direction of $y$ 's opinion than $y$ itself. We describe at the end of this section a framework in which this approach is more clearly justified, namely traffic modeling (pedestrians or cars). This section also aims at highlighting the deep differences between classical mechanical systems of the Hamiltonian type, which follow in particular the Law of Action-Reaction and conserve momentum, and the propagation of a non-conservative quantity on a directed network. 
We simply write than the "opinion velocity" $d u / d t$ relaxes to the expression given by the first-order model, with some characteristic time $\tau>0$ :

$$
\frac{d^{2} u}{d t^{2}}+\frac{1}{\tau}\left(\frac{d u}{d t}+\frac{1}{\eta} A u\right)=0
$$

It appears as a discrete version of a standard Partial Differential Equation, namely the weakly damped wave equation, which can be written

$$
\frac{\partial^{2} u}{\partial t^{2}}+\frac{1}{\tau}\left(\frac{\partial u}{\partial t}-\frac{L^{2}}{\eta} \Delta u\right)=0
$$

where the constant $L^{2}$, homogeneous to a squared distance, has been added in order to preserve homogeneity, and where $u$ is now a scalar function. Note that, in this setting, the wave speed is $L / \sqrt{\eta \tau}$.

Indeed, the matrix $A$ has some similarities with a Laplace operator (more precisely with its opposite): the entries of its rows sum up to 0, diagonal elements are nonnegative, and extra-diagonal elements are nonpositive, which ensures the maximum principle. Considering this wave equation in a bounded, connected domain, with homogeneous Neuman boundary conditions, the $-\Delta$ operator admits an infinite family of eigenvalues $0=\mu_{0}<\mu_{1} \leq \mu_{2}, \ldots, \mu_{n} \rightarrow+\infty$, with associated eigenvectors $w_{0}, \ldots, w_{n}, \ldots$ The wave equation 29 can be projected along any of these eigenmodes $k \geq 1$, which yields a second order ODE of the spring-mass type:

$$
\frac{d^{2} \hat{u}_{k}}{d t^{2}}+\frac{1}{\tau} \frac{d \hat{u}_{k}}{d t}+\frac{L^{2} \mu_{k}}{\eta \tau} \hat{u}_{k}=0
$$

where $\hat{u}_{k}=\hat{u}_{k}(t)$ is the coefficient of the full solution $u$ associated to the $k$-th mode $\left(u(x, t)=\sum u_{k}(t) w_{k}(x)\right)$. For any $\mu_{k}>0$, this equation has two roots with negative real parts, which expresses asymptotic stability (pure damping or damped oscillations, see details below).

Now consider our non-symmetric discrete wave equation (28), we aim at showing that, in spite of the stabilizing character of $-A$ (the eigenvalues of $A$ have a nonnegative real part), the inertial version of the evolution model may develop instabilities. We shall assume here that the matrix $A=I-K$ is diagonalizable, with eigenelements $\left(\mu_{k}, W_{k}\right)_{1 \leq k \leq N}$. For any eigenvector $W$ with associated eigenvalue $\mu$, we can build solutions of 28 of the form

$$
t \mapsto e^{\lambda t} W
$$

where $\lambda$ is a root of the characteristic equation

$$
\lambda^{2}+\frac{1}{\tau} \lambda+\frac{1}{\tau \eta} \mu=0 .
$$

We thus obtain

$$
\lambda^{ \pm}=\frac{1}{2 \tau}\left(-1 \pm \sqrt{1-4 \frac{\tau}{\eta} \mu}\right) .
$$

Case of a real spectrum

Let us first consider the situation where the spectrum of $A$ lies on the real line, which amounts to assume an underlying gradient flow structure of the first-order evolution problem (see Proposition 4.2). In this case, the system projected along any eigendirection $W$ takes the form of a damped linear oscillator (30) (spring-mass system). If one denotes by $T$ the translation $z \in \mathbb{C} \mapsto z+1$ and $Q: z \in \mathbb{C} \mapsto z^{2}$, the pair of eigenvalues associated to $\mu \geq 0$ can be written

$$
\lambda^{ \pm}=\frac{1}{2 \tau} T^{-1} Q^{-1} T\left(-4 \frac{\tau}{\eta} \mu\right) .
$$

The two possibilities are represented in Figure 6. Depending on whether $-4 \mu \tau / \eta<-1$ or not, we shall have damped oscillations (case $\mu_{1}$ on the figure, with $\lambda_{1}^{ \pm}$have non-zero imaginary parts) or pure damping (case $\mu_{2}$ 


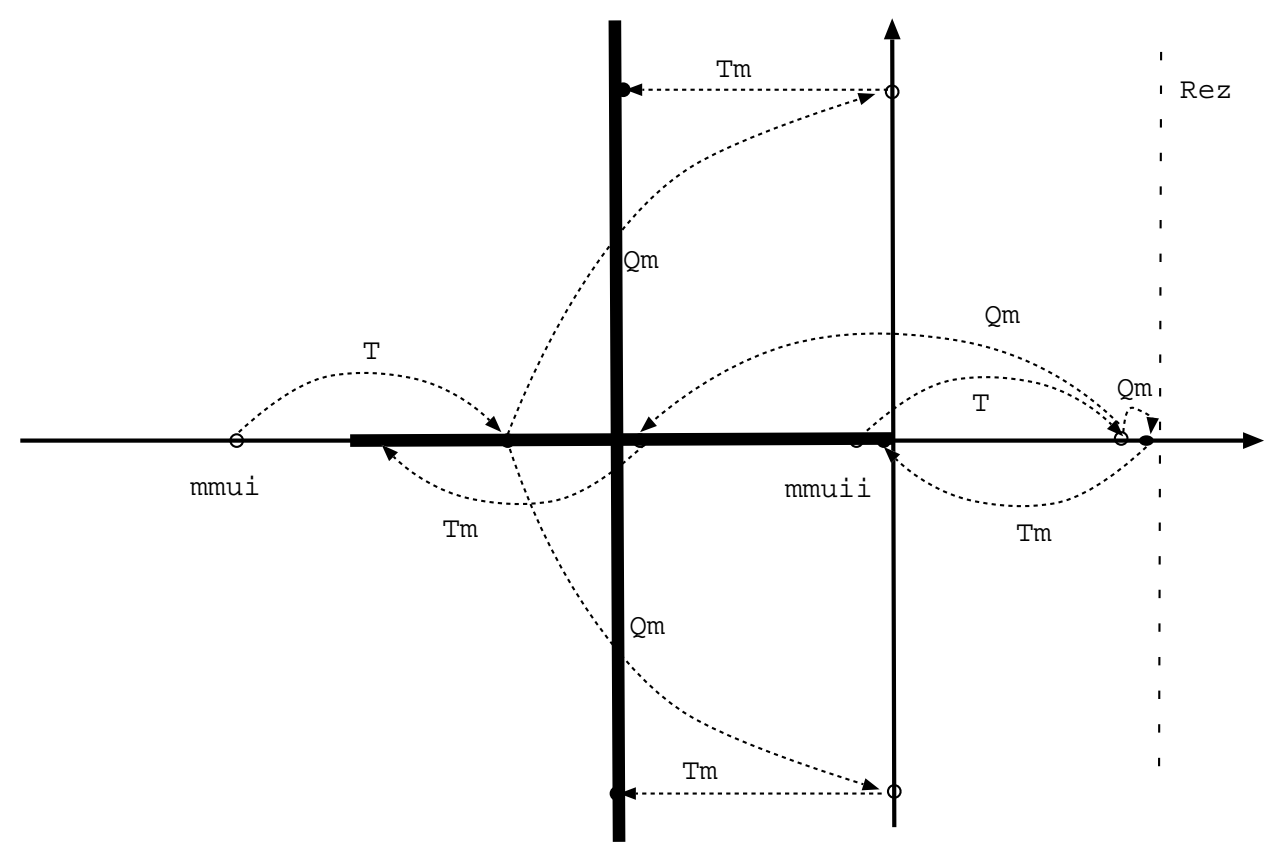

FiguRE 6. Damped oscillations and pure damping

on the figure, where $\lambda_{2}^{ \pm}$are real and negative). The zone where all the $\lambda^{ \pm}$'s lie is the bold cross represented in Figure 6.

\section{General case}

In the general situation, the evolution equation along any $A$ eigendirection can be seen as a damped oscillator equation with a possibly complex stiffness. We shall see that the complex character of some eigenvalues rules out the asymptotic stability, i.e. some $\lambda$ 's may have positive real parts. This question can be formulated by means of the mappings $T$ and $Q$ introduced previously: The system is unstable as soon as

$$
T^{-1} Q^{-1} T\left(-4 \frac{\tau}{\eta} S_{p}(A)\right) \cap[\Re(z)>0] \neq \emptyset,
$$

where $S_{p}(A)$ is the spectrum of $A$. The previous condition is equivalent to

$$
-4 \frac{\tau}{\eta} S_{p}(A) \cap T^{-1} Q([\Re(z)>1]) \neq \emptyset .
$$

The set $T^{-1} Q([\Re(z)>1])$ can be explicitly described. Its boundary is

$$
\left\{(1+i t)^{2}-1=-t^{2}+2 i t, t \in \mathbb{R}\right\},
$$

which is the parabola $\left[x=-y^{2} / 4\right]$. The set $T^{-1} Q([\Re(z)>1])$ is then $\left\{z=x+i y, x \geq-y^{2} / 4\right\}$.

We may now express the stability property:

Proposition 8.1. Let $A=I-K$ be given, with $K$ stochastic matrix. The evolution problem 28) is stable as soon as

$$
-4 \frac{\tau}{\eta} S_{p}(A) \subset\left[x \leq-y^{2} / 4\right] .
$$




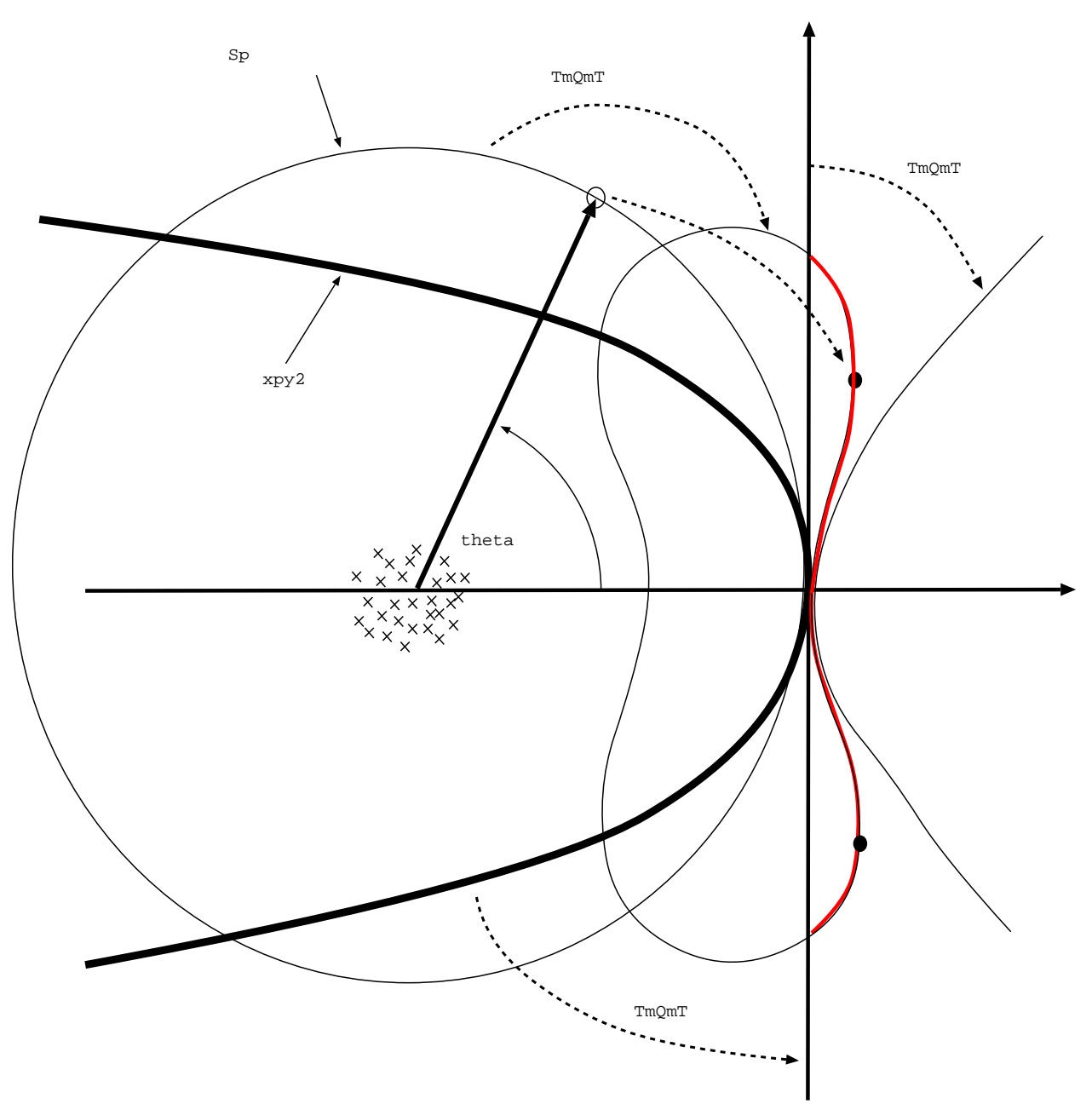

Figure 7. Stability parabola

In particular, if $\tau \leq 2 \eta$, it is stable.

If at least one eigenvalue of $A$ does not verify the inclusion above, the problem is unstable.

Proof. The stability condition has been established above. The condition on $\tau / \eta$ comes from the fact that $A=I-K$, where $K$ is stochastic, so that the eigenvalues of $A$ lie in $1-C$, where $C$ is the unit disc. Let us introduce $\alpha=4 \tau / \eta$. The disc $-4 \tau / \eta(1-C)=-\alpha(1-C)$ is represented in Figure 7 . It is contained in the convex hull of the stability parabola as soon as its radius is not larger than 2 , which is the reciprocal of the curvature of the parabola at the origin.

In the worst-case scenario in terms of stability, eigenvalues of $K$ distribute over the unit circle, so that the spectrum of $A=I-K$ distribute over the circle centered at 1, with radius 1 (such a situation can occur in the case of a pure cycle as presented in Section 4 4 . The circle obtained by multiplying it by $-4 \tau / \eta$ is represented in Figure 7. Its image under $T^{-1} Q^{-1} T$ is the quartic represented in the same figure. Unstable eigenvalues of the inertial problem are represented in red (see more details below) . 


\section{Most unstable mode}

A natural question arises: to which eigenmode of $A$ does the most unstable mode of the inertial system correspond? We shall consider here the case when the spectrum of $A=1-K$ is distributed along the circle $1-C$, centered at 1 , with radius 1 , where $C$ is the unit circle in $\mathbb{C}$. The circle $-\alpha(1-C)$ is represented on figure 7, with $\alpha=4 \tau / \eta$.

The approach which we propose consists in identifying the zone on which eigenvalues of the inertial system, to locate on this zone the most unstable mode, and to investigate to which mode of $A$ it corresponds. The set $T(-\alpha(1-C)) \in \mathbb{C}$ is the circle centered at $1-\alpha$, with radius $\alpha$, i.e. the set of $(\tilde{x}, \tilde{y})$ verifying

$$
(\tilde{x}-1+\alpha)^{2}+\tilde{y}^{2}-\alpha^{2}=0 .
$$

A complex $z=x+i y$, with square $z^{2}=x^{2}-y^{2}+2 i x y$, is then in $Q^{-1} T(-\alpha(1-C))$ if and only if

$$
f(x, y)=\left(x^{2}-y^{2}-1+\alpha\right)^{2}+4 x^{2} y^{2}-\alpha^{2}=0 .
$$

The solution set is a quartic curve, which can be checked to delimit a convex domain for $\alpha \leq 2$. It has two connected components for $0 \leq \alpha<1 / 2$. For $\alpha=1 / 2$ it is a Bernoulli Lemniscate, for $\alpha=1$ it coincides with the unit disc, and for $\alpha=2$, it has a stadium-like shape. Convexity is lost for $\alpha>2$, which corresponds to the emergence of instabilities. The 8-shaped closed curve in Figure 7 represented on of those curves, translated by -1 , for $\alpha>2$. The equation of this curve is obtained by replacing $x$ by $x+1$ in the expression of $f(x, y)$ above. The most unstable modes correspond to the maximal real part, it corresponds to the black dots in the figure. At these points, the gradient of $f$ is directed along the real $x$-axis, therefore

$$
\partial_{y} f(x, y)=4 y\left(x^{2}+y^{2}-+1-\alpha\right)^{2}=0 \Longrightarrow y^{2}=-x^{2}-1+\alpha .
$$

From the equation of the curve itself, it comes $x^{2}=\alpha^{2} / 4(\alpha-1)$. We now aim at locating the antecedent (by $Q$ ) of this point on the circle $T(-\alpha C)$. Coming back to the variables $(\tilde{x}, \tilde{y}))$, this point corresponds to $\tilde{x}=x^{2}-y^{2}$. Keeping in mind that we are considering the figure translated by 1 , the radial vector (bold arrow in Figure 7 ) has a first coordinate equal to

$$
\tilde{x}-1+\alpha=x^{2}-y^{2}-1+\alpha=2 x^{2}=\frac{\alpha^{2}}{2(\alpha-1)} .
$$

Since the radius of the circle is $\alpha$, the cosine of angle $\theta$ is $(\tilde{x}-1+\alpha) / \alpha$, which yields

$$
\theta=\arccos \left(\frac{\alpha}{2(\alpha-1)}\right)
$$

For $\alpha$ slightly greater than 2 (emergence of instabilities), $\theta$ is close to 0 , which corresponds to low frequencies. The typical situation is that of a $N$ cycle, with $N$ large, and a mode at minimal frequency, i.e. $(\exp (2 i \pi \ell / N))_{\ell}$ (a single oscillation over the whole cycle). For larger values of $\alpha$, the angle $\theta$ quickly approaches its asymptotic value, that is $\arccos (1 / 2)=\pi / 3$. For a $N$-cycle, this corresponds to an eigenvector of the form $(\exp (2 i \pi k \ell / N))_{\ell}$ with $k=N / 6$, which corresponds to oscillations of period 6 . It reveals that, in the context we considered, the configuration that is the most likely to lead to instabilities is the 6-cycle.

Remark 8.2. The situation where the spectrum of $K$ lies on the unit circle corresponds to pure cycles. Those cycles are not very realistic in real-life social network. Yet, they natively appear in another context, which also involves "agents" of another kind: the modeling of car (or pedestrian) traffic. Consider a set of cars in a periodic road, the positions of which are denoted by $q_{1}, \ldots, q_{N}$. Consider a behavior function $\beta(\cdot)$, which maps distances to speeds, i.e. a driver at a distance $w$ to the next car will tend to adopt a velocity $\beta(w)$. The function 
is typically increasing from 0 to some limit value, and concave. If one accounts for the inertia of the vehicle and the reaction time of driver, it is natural to write the model as

$$
\ddot{q}_{i}=\frac{1}{\tau}\left(\beta\left(q_{i+1}-q_{i}\right)-\dot{q}_{i}\right)
$$

Expressed in terms of distances $w_{i}=q_{i+1}-q_{i}$ (with periodicity), the model takes the form

$$
\ddot{w}_{i}=\frac{1}{\tau}\left(\beta\left(w_{i+1}\right)-\beta\left(w_{i}\right)-\dot{w}_{i}\right)
$$

This problem admits a trivial equilibrium point, with the cars uniformly distributed on the road (distance $w_{e q}$ ), all driving at speed $\beta\left(w_{e q}\right)$. Investigating the linear stability of this equilibrium point, we obtain the second order linear equation

$$
\ddot{w}+\frac{1}{\tau} \dot{w}+\frac{1}{\eta \tau} A w=0
$$

with $A=I-K$, where $K$ is a the stochastic matrix associated to the leftward shift (the associated "random" walk is in fact purely deterministic), i.e. it exactly corresponds to the cycle which we considered previously. The stability analysis detailed above shows that instabilities are likely to appear, and that the most unstable mode correspond to a period of 6 in terms of cars (emergence of Stop and Go waves, see e.g. [52]). We refer the reader to 44 for further details on this traffic model, and its stability analysis.

\section{General case}

In the general setting, eigenvalues of $K$ lie in the unit disc, possibly far from the circle for most of them (see additional details below). Spotting the most unstable mode necessitates an alternative method. It can be carried out by simply considering the images of the sets $[\Re(z)>r]$ by $T^{-1} Q T$, for $r \geq 0$. For $r=0$, we recover the parabola represented in Figure 7, which delimits the stability domain, i.e. the model is unstable as soon a $-\alpha S_{p}(A)$ lies outside the convex domain $D_{0}=\left[x+y^{2} / 4 \leq 0\right]$. For larger values of $r$, a straightforward computation leads to a family of domains delimited by parabolas:

$$
D_{r}=\left\{z=x+i y, x+\frac{4 y^{2}}{(1+r)^{2}}-(1+r)^{2}+1 \leq 0\right\} .
$$

The most unstable mode, i.e. the mode which give the largest real part to an eigenvalue of the global system, can be obtained as follows: the smallest $r>0$ such that $-\alpha S_{p}(A) \subset D_{r}$ is the largest real part. This minimum is realized by at least one (in fact two, since complex eigenvalues of the real matrix $A$ come by pairs of conjugates) eigenvalue of $A$, i.e. $\mu \in S_{p}(A)$ such that $-\alpha \mu$ lies in the boundary of $D_{r}$, and any such eigenmode is maximal in terms on instability.

\section{Spectrum of stochastic matrices}

Let us end this section by some comments on what can be expected concerning the spectrum, and consequently stability properties of the inertial model, of a stochastic matrix associated to a social network. We established that instabilities where likely to occur in the situation where the network contains pure cycles (like in the caricatural situation presented in Section A. But we have to admit that, except for very special situations like traffic-like problems in a periodic setting (see Remark 8.2), or Chinese Whispers game, pure 6-cycles have little chance to be observed in real life social networks. Another extreme situation corresponds to charismatic networks, which obey some sort of Law of Action Reaction, so that the matrix $K$ has only real eigenvalues. In this situation, the inertial problem evolves like a hamiltonian system (e.g. a spring mass system) with damping, and asymptotic stability prevails. The generic situation for social networks can be expected to lie between those extreme situations. A very general result has actually been proved recently (see [16]), for random matrices 
which are built as follows: consider $\left(X_{i j}\right)_{1 \leq i, j \leq N}$ identically distributed and independent nonnegative random variables with bounded density, expected value $m$, and finite variance $\sigma^{2}$, and the associated random stochastic matrix $K$ of order $N$ defined by

$$
K_{i j}=\frac{X_{i j}}{\sum_{\ell} X_{i \ell}} .
$$

The spectrum of $K$ tends to spread uniformly over the disc of radius $1 / \sqrt{N}$ times a correcting factor $\sigma / m$. This distribution is illustrated in Figure 7 by the cloud of little crosses gathered around the center of $-\alpha(1-C)$ ). Note that this concentration of eigenvalues of $K$ around the origin tends to keep $-\alpha S_{p}(1-K)$ away from the boundary of the stability parabola $D_{0}$, thereby contributing to stabilize the inertial system. Yet, as soon as an eigenvalue of $K$ is not purely real, the inertial problem will eventually become unstable for sufficiently large values of $\alpha$, i.e. large values of $\tau / \eta$, and little is known about large deviations with respect to the asymptotic behavior mentioned above. More precisely, the fact that most of those random stochastic matrices may have individual eigenvalues close to the unit circle $C$ may impair the stabilizing effect of generic concentration, without contradicting the asymptotic results presented above. Besides, the family of matrices defined above is not typical of real-life social networks, and a full understanding of spectral properties of large realistic social network is still missing. We refer to 47] for a detailed account of typical properties of social networks, and to [58] for a recent study of real-life dynamic networks.

\section{FUZZY OPINION, PROPAGATION OF MEASURES}

The idea we aim at developping here was already present in the seminal paper 24. The individual opinion is described as a probability measure over some feasible set, to account for the fact that some agents may not be so sure about their opinion, and the propagation affects those probability measures. More precisely the model is based, like in the previous sections, on a known set of coefficients $K_{x y}$ to quantify influences. Assuming that agents form opinions about the value of some parameter $u$ in a (measured) space $\Omega$, the opinion of an individual $x$ is described by a probability measure $\rho_{x}$ over $\Omega$ : for any set $A \subset \Omega$, the mass given by $\rho_{x}$ to $A$, namely $\int_{A} \rho_{x}(u) d u$ is the degree of belief of agent $x$ that the value of $u$ belongs to $A$. If $\rho_{x}$ is a Dirac mass, i.e. a probability measure concentrated on a single point $\bar{u} \in \Omega$, it means that agent $x$ is fully convinced that the value of the parameter $u$ is $\bar{u}$.

\subsection{Degroot original evolution model}

The model proposed in 24 is based on a relation similar to (2):

$$
\rho_{x}^{k+1}=\sum_{x \rightarrow y} K_{x y} \rho_{y}^{k}
$$

In spite of the formal analogy, the model is actually quite different from (2) in terms of opinion propagation. To fix the ideas, consider the case when the opinion pertains to the value of some sensitive quantity $u$ (like the mean temperature rise in 20 years). Now consider an agent $x$ that equally points to two influencers $y_{0}$ and $y_{1}$, the opinion of which strongly differ (say, 0 for $y_{0}$ and 1 for $y_{1}$ ), each of them being fully self confident, so that the associated measures are Dirac masses. The model above builds the opinion of $x$ as the half sum of Dirac masses, so that $x$ ends up considering that the value of $u$ is either 0 or 1 , with probability $1 / 2$ (see Figure 8 (b) for a case where the influencers are quite certain about their opinion). In this setting, the outcome is obviously unrealistic. It is more appropriate to consider that $x$ should be led to consider that the real value lies between 0 and 1. A first attempt in this direction is proposed in the next section. 


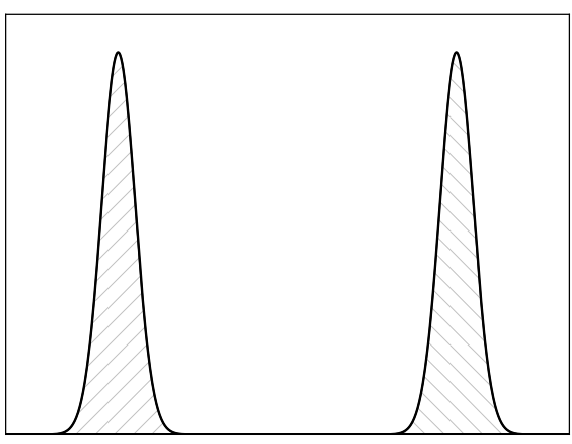

(a) Two distributions

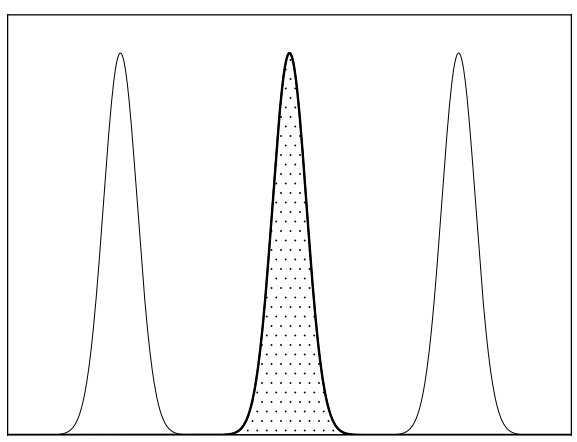

(c) Wasserstein interpolation

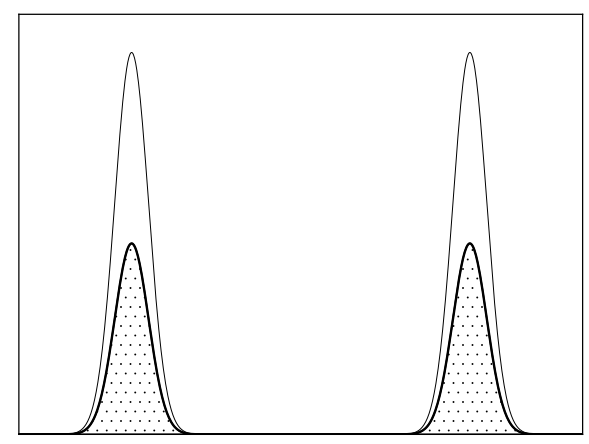

(b) Linear interpolation

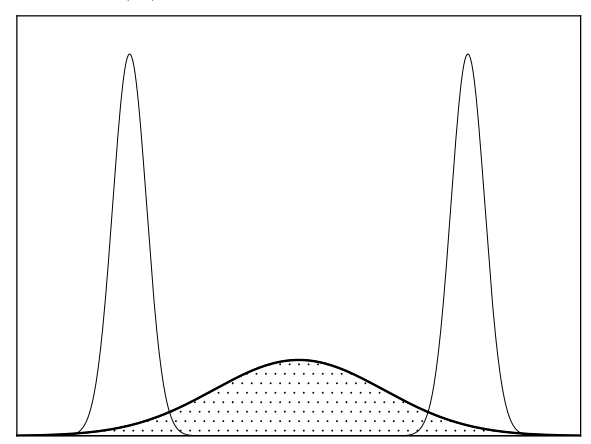

(d) Fisher interpolation

FiguRE 8. Barycenters of two peaked Gaussian distributions: linear, Wasserstein, and Fisher.

\subsection{Wasserstein metric}

In order to circumvent the non-realistic character of the model presented above, which consists in interpolating in a linear manner by using the straight addition of densities, it may be tempting to use an alternative definition of this interpolation. This alternative way can be defined within the framework of optimal transportation (see e.g. 57]). Since this strategy still suffers from important flaws in terms of modeling, we shall present it quite shortly, as an intermediary step to reach the approach presented in the next section. The basic idea consists in estimating distances between probability measures by means of optimal transportation. More precisely, in the one-dimensional context, we consider two measures $\rho_{0}$ and $\rho_{1}$ (supported in a compact interval $I$, to fix the ideas), and we denote by $\Pi$ the set of probability measures $\gamma$ on $I \times I$ with marginals $\rho_{0}$ and $\rho_{1}$, respectively. For any given cost $c(x, y)$ (which quantifies the cost to move one unit of "mass" from $u$ to $v$ ), one can define the total transportation cost from $\rho_{0}$ to $\rho_{1}$

$$
C\left(\rho_{0}, \rho_{1}\right)=\inf _{\gamma \in \Pi} \int_{I \times I} c(u, v) d \gamma(u, v) .
$$

For the quadratic cost $c(u, v)=(v-u)^{2}$, the quantity $W_{2}\left(\rho_{0}, \rho_{1}\right)=C\left(\rho_{0}, \rho_{1}\right)^{1 / 2}$ can be shown to be a distance on the space of probability measures supported over $I$, the so-called quadratic Wasserstein distance, and the infimum above is attained. A geodesic curve between any two measures can also be built (see the seminal paper [46]). In the case when the transport plan is actually a map, i.e. if the infimum above can be written

$$
\int_{I} c(u, T(u)) d \rho_{0}(u)
$$


where $T$ pushes forward $\rho_{0}$ to $\rho_{1}$, then the geodesic curve is

$$
\rho_{t}=(I-t(T-I))_{\sharp} \rho_{0},
$$

that is, for any $t \in[0,1]$, the image measure of $\rho_{0}$ by the mapping $u \mapsto(1-t) u+t T(u)$. For two Dirac masses at $u_{0}$ and $u_{1}, \rho_{t}$ is the Dirac mass at $(1-t) u_{0}+t u_{1}$ (see Figure 8 (c) for the case $t=1 / 2$ with peaked Gaussian measures). An evolution model based on this approach could be built as follows (in the discrete setting), by keeping the underlying framework of a set $V$ of vertices (agents) together with weights $\left(K_{x y}\right)$. The problem can be written

$$
\rho^{k+1}=\operatorname{Bar}\left(\left(\rho_{y}^{k}, K_{x y}\right)_{x \rightarrow y}\right),
$$

which is the (Wasserstein) barycenter of the $\rho_{y}^{k}$,s with weights $K_{x y}$, for all the agents $y$ that influence $x$. Such a barycenter is indeed well-defined for probability measures defined on a real interval: specifically, $\rho_{x}^{k+1}$ is defined from the $\rho_{y}^{k}$ 's as the minimizer of

$$
\sum_{x \rightarrow y} K_{x y} W_{2}\left(\rho, \rho_{y}^{k}\right)^{2}
$$

We shall not study further this approach, because it presents two serious flaws. Let us start by a mathematical difficulty: in dimensions higher than 1, the Wasserstein space of probability measures has the wrong curvature (namely it is positively curved) which makes the study of barycenters quite involved 22 48. The second problem is deeper, since it affects the very core of the model: consider again the case of a agent $x$ equally influenced by two agents $y_{0}$ and $y_{1}$, the opinion of which are characterized by Dirac masses at 0 and 1 , respectively. The opinion of $x$ is, according to the previous model, represented by $\delta_{1 / 2}$, i.e. a Dirac mass at $1 / 2$. This behavior seems more reasonable that $\left(\delta_{0}+\delta_{1}\right) / 2$, but it is still not satisfactory. Indeed it would mean that the third agent is fully sure that the reality exactly lies at the mean between their influencers' opinions. In particular, $x$ considers that there is a probability zero than one of the influencer may be right. It would be much more reasonable to expect that the new opinion would be indeed centered at $1 / 2$, but with some uncertainty, and to leave some chance that one of the influencers may be right. More generally, with the Wasserstein metric the evolutions of the mean opinion of an agent and its uncertainty about it are decoupled, which makes the introduction of uncertainties in the agent's judgment almost superfluous. A strategy to remedy to this effect is presented in the next section.

We mention nevertheless that the equilibrium configurations of such models have been investigated (for the case of a symmetric network) under the name of Wasserstein propagation and have found applications in statistics 60].

\subsection{Fisher metric}

As previously said about the 3 -agent toy problem proposed above, with the 2 fully convinced influencers, it would be natural to consider that the agent $x$ ends up by estimating the value around $1 / 2$, but with some uncertainty. The total self-confidence of the influencers has no reason to be contagious and propagate, especially in the case where their opinion strongly differ. We propose here an approach based on the so-called Fisher metric, which comes from the notion of relative entropy. We shall restrict it to the case of Gaussian densities. Other families of densities might be considered, but the rigidity induced by the choice of a well-defined parametric family is essential, as detailed in Remark 9.8 . The idea is the following: consider a probability density $\rho=\rho(u)$ over some measurable set $\Omega$ (the set which contains all possible opinions), and a variation $\delta \rho$, such that $\rho+\delta \rho$ is still a probability density. The relative entropy of $\rho+\delta \rho$ with respect to $\rho$ (also called Kullback-Leibler divergence) writes

$$
\mathrm{KL}(\rho+\delta \rho \mid \rho)=\int_{\Omega}(\rho+\delta \rho) \log \left(\frac{\rho+\delta \rho}{\rho}\right) d u
$$


Note that the previous quantity is also the standard entropy of the density $\rho+\delta \rho$ with respect to $\rho$, measured against $\rho$ itself:

$$
\mathrm{KL}(\rho+\delta \rho \mid \rho)=\int_{\Omega} \frac{\rho+\delta \rho}{\rho} \log \left(\frac{\rho+\delta \rho}{\rho}\right) \rho d u
$$

Remark 9.1. (Relative entropy and measure of opinion discrepancy)

Measuring the difference between two densities in terms of relative entropy makes some sense in the context of opinions. In particular, if an agent has an opinion described by $\rho$, with $\rho$ almost vanishing in some zone $\omega$ of $\Omega$, it means that this agent considers as very unlikely that the value of $u$ could belong to $\omega$. Any opinion which gives a significant probability that $u$ may belong to $\omega$ can then be considered as very remote from $\rho$, which is exactly expressed by (34). More precisely, if $\delta \rho$ has a positive value on $\omega$, even a small value, KL divergence will converge to $+\infty$ when $\rho$ converges to 0 on $\omega$. Let us push this interpretation a little further: the non-symmetry of the KL divergence makes some sense in the context of opinion: if an agent gives to $\omega$ some (small) positive probability, the opinion which rules out $u \in \omega$ is not so far away from the initial one, which is again reflected by (34). In other words, the way to measure discrepancy provided by the relative entropy is consistent with the asymmetry which is natural in the world of opinions encoded by probabilities: $\epsilon>0$ is far away from 0 , whereas 0 is actually not so far away from $\epsilon$.

The relative entropy of $\rho+\delta \rho$ with respect to $\rho$ can be expressed as follows, owing to the fact that $\int \delta \rho=0$,

$$
\mathrm{KL}(\rho+\delta \rho \mid \rho)=\int_{\Omega}(\rho+\delta \rho)\left(\frac{\delta \rho}{\rho}-\frac{(\delta \rho)^{2}}{2 \rho^{2}}+o\left((\delta \rho)^{2}\right)\right) d u \sim \frac{1}{2} \int_{\Omega} \frac{(\delta \rho)^{2}}{\rho}=\frac{1}{2} \int_{\Omega}(\delta \log \rho)^{2} \rho d u
$$

Now consider a family of probability densities $\left(\rho_{\theta}\right)$ parametrized by $\theta=\left(\theta_{1}, \ldots, \theta_{p}\right)$, in some parameter space $\Theta$. In this parametric context, the previous expansion yields

$$
\mathrm{KL}\left(\rho_{\theta+\delta \theta} \mid \rho_{\theta}\right)=\langle I \delta \theta \mid \delta \theta\rangle+o\left(\delta \theta^{2}\right)
$$

where $I$ is the so-called Fisher information matrix:

$$
I=\left(I_{i j}\right)_{1 \leq i, j \leq p}=\left(\int_{\Omega}\left(\frac{\partial \log \rho_{\theta}}{\partial \theta_{i}}\right)\left(\frac{\partial \log \rho_{\theta}}{\partial \theta_{j}}\right) \rho_{\theta} d u\right)_{1 \leq i, j \leq p} .
$$

This canonically defines a Riemannian metric, i.e. a expression of the square length of an infinitesimal variation in the parameter space

$$
d \theta^{2}=\langle I d \theta \mid d \theta\rangle
$$

Consider now a smooth curve in this space of parametrized probability densities, between $\rho_{\theta_{0}}$ and $\rho_{\theta_{1}}$. We identify it with the corresponding curve in the parameter space $\Theta$, that is $t \in[0,1] \mapsto \theta(t)$. The length of this curve according to the metric we consider is then defined as

$$
\ell=\int_{0}^{1}\langle I \dot{\theta} \mid \dot{\theta}\rangle^{1 / 2} d t
$$

In the case when every two densities can be connected by at least such a curve with finite length, it makes it possible to define a geodesic distance as

$$
d_{F}\left(\rho_{\theta_{0}}, \rho_{\theta_{1}}\right)=\inf _{\theta \in \Xi} \int_{0}^{1}\langle I \dot{\theta} \mid \dot{\theta}\rangle^{1 / 2} d t
$$

where $\Xi$ is the set of all smooth curves $t \in[0,1] \mapsto \theta(t) \in \Theta$ which connect $\theta_{0}$ and $\theta_{1}$. One may now wonder whether such a setting makes it possible to properly define barycenters. More precisely, considering parameters 


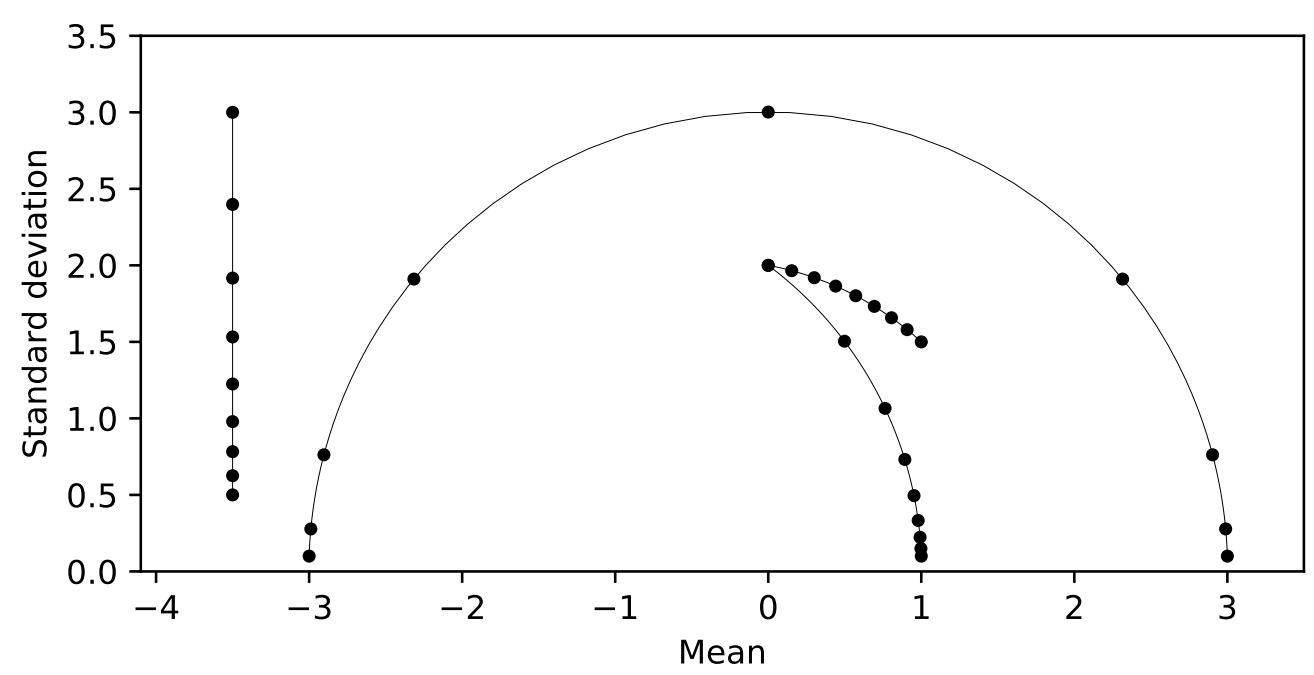

Figure 9. In the Poincaré half-plane, where the horizontal axis stands for the mean and the vertical axis for the standard deviation (up to a factor $\sqrt{2}$ ), four geodesics are displayed with equidistant points (for the Fisher distance) on it. Notice that the points are not equidistant for the usual Euclidean distance.

$\theta_{1}, \ldots, \theta_{p}$ together with positive weights $K_{1}, \ldots, K_{p}$, with $\sum K_{i}=1$, does the problem

$$
\min _{\theta \in \Theta} \sum_{i=1}^{p} K_{i} d_{F}\left(\rho_{\theta}, \rho_{\theta_{i}}\right)^{2}
$$

admit a unique solution $\bar{\theta}$ ? If it does, we shall express it in terms of parameters or densities, depending on the context:

$$
\bar{\theta}=\operatorname{Bar}\left(\left(\theta_{i}, K_{i}\right)_{1 \leq i \leq p}\right) \quad \text { or } \rho=\rho_{\bar{\theta}}=\operatorname{Bar}\left(\left(\rho_{\theta_{i}}, K_{i}\right)_{1 \leq i \leq p}\right) .
$$

Remark 9.2. (Geodesics and barycenters)

We mention that, in the case of two densities, parametrized by $\theta_{0}, \theta_{1}$, if there is a unique curve $t \mapsto \theta(t) \in \Xi$ which is optimal in (36) and which is traveled at constant-speed (i.e. such that $\langle I \dot{\theta} \mid \dot{\theta}\rangle$ is constant), then for any $K \in[0,1]$,

$$
\operatorname{Bar}\left(\left(\theta_{0}, 1-K\right),\left(\theta_{1}, K\right)\right)=\theta(K) .
$$

Such a curve $\theta$, which is nothing else than a constant-speed geodesic between $\theta_{0}$ and $\theta_{1}$, provides a way to interpolate between these two parameters. The notion of barycenter can be seen as a way to generalize this notion of interpolation between more than just two parameters.

In the case when barycenters are defined without ambiguity, one may define an evolution problem which mimics (2) as

$$
\theta_{x}^{k+1}=\operatorname{Bar}\left(\left(\theta_{y}^{k}, K_{x y}\right)_{x \rightarrow y}\right) .
$$

Now consider the particular case of Gaussian densities over $\mathbb{R}$ :

$$
\rho_{\theta}(u)=\frac{1}{\sqrt{2 \pi} \sigma} e^{-\frac{(u-m)^{2}}{2 \sigma^{2}}},
$$



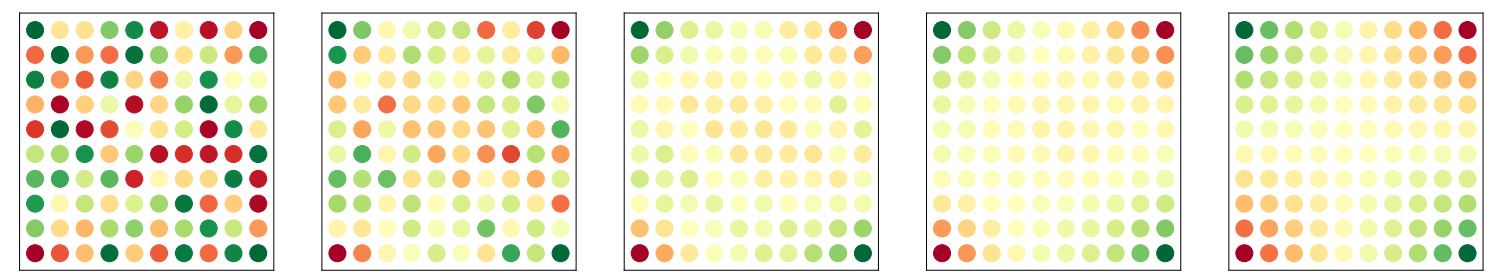

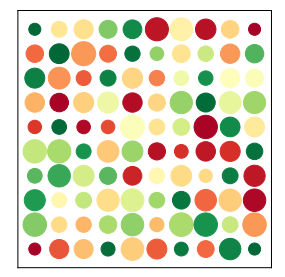

$k=0$

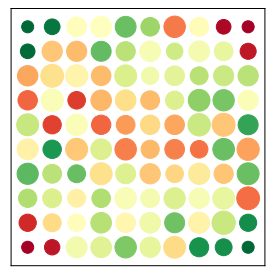

$k=1$

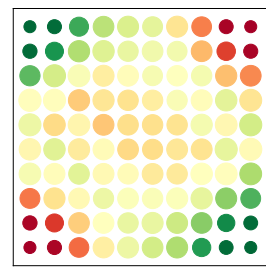

$k=5$

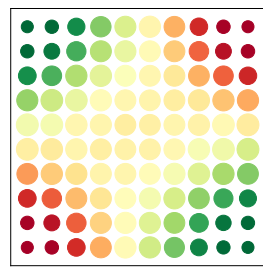

$k=10$

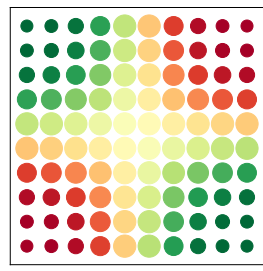

$k=30$

FiguRE 10. Example of propagation of opinion, both for the linear evolution model (2) (top row) and the one with the Fisher metric (37) (bottom row). For the Fisher metric, we used the conformal metric of the Poincare half plane $(\kappa=1)$, not the distorted one. The network is a Cartesian grid, with four influencers located in the four corners. Each agent (except the influencers) listens equally to each of its neighbors, and slightly to himself. The color of the square (from red to green) indicates the mean value of the opinion, while the size (for the bottom row) is proportional to the standard deviation, i.e. the inverse of their degree of confidence. One can observe that the Fisher evolution leads to an equilibrium situation (roughly reached for $k=30$ ) which is more polarized than for the linear evolution.

parametrized by $\theta=(m, \sigma) \in \Theta=\mathbb{R} \times(0,+\infty)$. Straightforward computations (see e.g. [23]) lead to a explicit expression of the Riemannian metric

$$
\langle I d \theta \mid d \theta\rangle=\frac{1}{\sigma^{2}} d m^{2}+\frac{2}{\sigma^{2}} d \sigma^{2} .
$$

This is, up to the factor 2 in the second term, the metric associated to the so-called Poincaré half plane $\mathbb{H}^{2}$, see for instance Chapters 2 and 6 of [20, as well as 21 . We shall actually remove this factor 2 and consider the standard situation where $I$ is a scalar matrix, that is the identity multiplied by $1 / \sigma^{2}$, and we put off until the end of this section (see Remark 9.7) some comments on alternative choices, consisting in distorting the metric in the vertical direction. This metric endows the half plane $\mathbb{H}^{2}=\mathbb{R} \times(0,+\infty)$ of parameters with a non Euclidean metric, which is such that geodesics are either vertical segments or circular segments, more precisely portions of circles centered on the $m$ (horizontal) axis: see Figure 9. For the interested reader, we provide an analytical expression of the constant-speed geodesics, see Appendix B. Since this space has a negative curvature, the barycenter of an arbitrary number of points is well-defined (see Appendix B and Proposition B.10 for more details), and the discrete evolution problem (37) properly defines a family of densities $\left(\rho_{x}^{k}\right)_{x \in V}$ which can be expressed in terms of parameters $\left(\theta_{x}^{k}\right)_{x \in V}$, which are points in $\mathbb{H}^{2}$.

Actually, in the $\mathbb{H}^{2}$ setting, the evolution defined by (37) is even amendable to numerical computations. As this is not the main focus of these notes, we delay the description of the algorithm to compute barycenters until Appendix B. Figure 10 shows an example of the computation of such an evolution.

This setting enjoys some theoretical properties which give some further reasons to use this distance in the present context of opinion propagation. 
Proposition 9.3. Let $\theta_{0}=\left(m_{0}, \sigma_{0}\right)$ and $\theta_{1}=\left(m_{1}, \sigma_{1}\right)$ be two elements of the Poincaré half-plane $\mathbb{H}^{2}$, $K$ a fixed coefficient in $(0,1), K_{0}=1-K, K_{1}=K$, and the barycenter between $\theta_{0}$ and $\theta_{1}$. We assume that $m_{0}<m_{1}$.

$$
\theta=\operatorname{Bar}\left(\left(\theta_{i}, K_{i}\right)_{i=0,1}\right)=(m, \sigma)
$$

\section{It holds that}

(i) $m$ belongs to $\left(m_{0}, m_{1}\right)$ and $m-\left(K_{0} m_{0}+K_{1} m_{1}\right)$ has the same sign as $\sigma_{0}-\sigma_{1}$.

(ii) $\sigma>\min \left(\sigma_{0}, \sigma_{1}\right)$.

(iii) $m \longrightarrow m_{0}$ when $\sigma_{0}$ goes to 0 .

(iv) $m \longrightarrow m_{1}$ when $\sigma_{0}$ goes to $+\infty$.

Proof. Given Remark 9.2, from a mathematical point of view we are rather making a statement about constant speed geodesics in the Poincaré half plane. All the statements can be verified analytically with the formulas given in Appendix B, but in fact are quite intuitive geometrically.

Let us add some comments on the previous proposition, in terms of modelling:

(i) $m \in\left(m_{0}, m_{1}\right)$ simply express that the resulting mean opinion is an interpolation between the mean value of influencers, which reflects the consensual character of the model. Second inequality : the interpolated value does not identify with the standard (affine) one, more precisely it bends in the direction of the most convinced influencer: if $\sigma_{0}<\sigma_{1}$, it means that 0 is more convinced than 1 , and $m$ will be closer to $m_{0}$ than the standard barycenter.

(ii) If an agent is influenced by two persons who disagree, their degree of confidence is less (i.e. the standard deviation is larger) than that of the most convinced influencer. If one considers that the first agent is actually the one with parameter $\theta_{0}$ (i.e. $K_{0}$ is the self inertia of the influencee), it simply expresses that discussing with a less convinced person with another opinion does not only change the opinion, but reduces one's confidence in their own opinion. Actually, we will state a more general result in Remark 9.6 .

(iii) If an influencer becomes fully convinced, they become fully convincing, i.e. their opinion wins. If one considers than the influenced one is actually the one with parameter $\theta_{0}$, it expresses that a highly convinced individual (with a tiny standard deviation) is actually stubborn (their mind cannot be changed by someone more reasonably convinced). Mathematically, it expresses the fact that Dirac masses (i.e. measures for which $\sigma=0$ ) are at an infinite distance from all other points in $\mathbb{H}^{2}$ : stubborn individuals end up far away from the social network core (including from agents which share the same belief, with less self-confidence), while keeping a predominant ability to influence others.

(iv) If an influencer is poorly convinced, they lose the ability to convince anyone, and the influence power of their opinion vanishes.

Similarly to the evolution in the linear case, we can prove that, in the situation where everyone is connected to an influencer, the distribution of opinions, if it evolves according to (37), converges to an equilibrium. From a mathematical point of view, such a result has been proved in a much more general context in [63]: the evolution may take place not only a network, but on a arbitrary space endowed with a Markov semigroup, and the set $\mathbb{H}^{2}$ could be replaced by a non-positively curved space in the sense of Alexandrov. We will try to give a flavor of the proof of 63 by adapting it to our context. The main tool, which we will admit, is a version of Jensen's inequality suited to the geometry of $\left(\mathbb{H}^{2}, d_{F}\right)$.

Proposition 9.4. (Jensen's inequality)

Let $\left(K_{i}\right)_{i=1, \ldots n}$ be a collection of positive weights which sum to 1 . Take $\theta_{1}, \ldots, \theta_{n}$ and $\theta_{1}^{\prime}, \ldots, \theta_{n}^{\prime}$ two families of points in $\mathbb{H}^{2}$. Then

$$
d_{F}\left[\operatorname{Bar}\left(\left(\theta_{i}, K_{i}\right)_{i}\right), \operatorname{Bar}\left(\left(\theta_{i}^{\prime}, K_{i}\right)_{i}\right)\right] \leq \sum_{i=1}^{n} K_{i} d_{F}\left(\theta_{i}, \theta_{i}^{\prime}\right)
$$


This property is not straightforward and we will admit it, only referring to the appropriate reference. Notice that if $\mathbb{H}^{2}$ is replaced by the real line, and $d_{F}$ is replaced by the Euclidean distance, then the inequality is a simple consequence of standard Jensen's inequality and the fact that the Euclidean distance is a convex function.

Proof. As $\mathbb{H}^{2}$ endowed with the Fisher distance is a Hadamard manifold, the space $\left(\mathbb{H}^{2}, d_{F}\right)$ is a non-positively curved (NPC) space in the sense of Alexandrov [62, Proposition 3.1]. Hence the function $d_{F}$ is jointly convex, see 62, Corollary 2.5]. Thus we can apply Jensen's inequality for convex functions on NPC spaces, see 62, Proposition 6.1].

Proposition 9.5. Similarly to Proposition 1.10, assume that $\Gamma \neq \emptyset$ and that $V-\cdot \rightarrow \Gamma$. If $\theta$ is a solution of the evolution process (37), then $\theta^{k}$ converges as $k \rightarrow+\infty$ to $\theta^{\infty}$ which is an equilibrium point in the sense that for any $x \in V$,

$$
\theta_{x}^{\infty}=\operatorname{Bar}\left(\left(\theta_{y}^{\infty}, K_{x y}\right)_{x \rightarrow y}\right)
$$

Moreover, $\theta^{\infty}$ is uniquely determined by the values of $\theta^{0}$ on $\Gamma$.

Proof. As the reader will see, the proof relies on a reduction to the linear case. Everything starts with Jensen's inequality. If $\hat{\theta}, \tilde{\theta}$ are two solutions of the evolution process (37), using Proposition 9.4.

$$
d_{F}\left(\hat{\theta}_{x}^{k+1}, \tilde{\theta}_{x}^{k+1}\right) \leq \sum_{x \rightarrow y} K_{x y} d_{F}\left(\hat{\theta}_{y}^{k}, \tilde{\theta}_{y}^{k}\right)
$$

We take first $\tilde{\theta}_{x}^{k}=\vartheta$ where $\vartheta$ any given point in $\mathbb{H}^{2}$, independent on $k$ and $x$. Such a constant $\tilde{\theta}$ is obviously a solution to the evolution process. Then 40 reads as

$$
d_{F}\left(\hat{\theta}_{x}^{k+1}, \vartheta\right) \leq \sum_{x \rightarrow y} K_{x y} d_{F}\left(\hat{\theta}_{y}^{k}, \vartheta\right)
$$

By the maximum principle, it implies that the sequence $d_{F}\left(\hat{\theta}_{x}^{k+1}, \vartheta\right)$ is bounded independently on $k$ and $x$, by a constant which depends only on $\hat{\theta}^{0}$. In other words, the family $\left(\hat{\theta}_{x}^{k}\right)_{k, x}$ belongs to a bounded set for the Fisher distance.

Now take $\hat{\theta}$ and $\tilde{\theta}$ two solutions of the evolution process sharing the same values on $\Gamma$. By (40), the real-valued sequence $u$ defined by $u_{x}^{k}=d_{F}\left(\hat{\theta}_{x}^{k}, \tilde{\theta}_{x}^{k}\right)$ is a subsolution of the linear evolution process (2). Moreover $u^{k}$ vanishes on $\Gamma$ for any $k \geq 0$. Using Proposition 1.13 , we deduce that there exists $\left(r_{k}\right)_{k \in \mathbb{N}}$ a sequence depending only on the network and tending to 0 such that, for any $k$ and $x$,

$$
u_{x}^{k}=d_{F}\left(\hat{\theta}_{x}^{k}, \tilde{\theta}_{x}^{k}\right) \leq r_{k}\left(\max _{y} d_{F}\left(\hat{\theta}_{y}^{0}, \tilde{\theta}_{y}^{0}\right)\right) .
$$

Now, take $\theta^{k}$ a solution of the evolution process (37). We also fix $\ell$ an integer. We set $\hat{\theta}^{k}=\theta^{k}$ and $\tilde{\theta}^{k}=\theta^{k+\ell}$. Using the estimate above and the fact that $d_{F}\left(\theta_{x}^{k}, \vartheta\right)$ is bounded uniformly in $k$ and $x$, we deduce that for any $k$ and $x$

$$
d_{F}\left(\theta_{x}^{k}, \theta_{x}^{k+\ell}\right) \leq C r_{k}
$$

where $C$ can be taken for instance equal to $2 \max _{y} d_{F}\left(\theta_{y}^{0}, \vartheta\right)$.

Hence, we deduce that $\left(\theta_{x}^{k}\right)_{k \in \mathbb{N}}$ is a Cauchy sequence for the Fisher metric for any $x \in V$, it implies that it converges to some $\theta_{x}^{\infty}$. Using for instance [62, Theorem 6.3] to justify the continuity of the barycenter with respect to the weights, we can pass to the limit in (37) to get (39). Eventually, if $\hat{\theta}$ and $\tilde{\theta}$ are two solutions of the evolution process sharing the same boundary conditions, passing to the limit $k \rightarrow+\infty$ in 41 , we get that $\hat{\theta}^{\infty}=\tilde{\theta}^{\infty}$, which shows that the limit depends only on the values of $\theta^{0}$ on $\Gamma$. 
Remark 9.6. Now that we have stated Jensen's inequality, let us push further point (ii) of Proposition 9.3 . Indeed, thanks to the explicit formulas (44) and (45), one can check easily that the function $\theta=(m, \sigma) \mapsto 1 / \sigma$ is convex, i.e. convex along geodesics in $\mathbb{H}^{2}$. As a consequence, Jensen's inequality [62, Proposition 6.1] reads, for points $\theta_{1}, \theta_{2}, \ldots, \theta_{n}$ in $\mathbb{H}^{2}$ and nonnegative weights $K_{1}, K_{2}, \ldots, K_{n}$ which sums up to 1 : if $\theta=(m, \sigma)$ is the barycenter of the $\theta_{i}$ 's with weights $K_{i}$ 's then

$$
\frac{1}{\sigma} \leq \sum_{i=1}^{n} \frac{K_{i}}{\sigma_{i}} .
$$

As a consequence, if we look at our evolution process (37), denoting $\theta_{x}^{k}=\left(m_{x}^{k}, \sigma_{x}^{k}\right)$,

$$
\frac{1}{\sigma_{x}^{k+1}} \leq \sum_{x \rightarrow y} \frac{K_{x y}}{\sigma_{y}^{k}}
$$

If we remember that $1 / \sigma_{x}^{k}$ stands for the confidence of agent $x$ at time $k$ for their opinion, then we see that along the evolution process the confidence of an agent tends to be smaller than the one of their neighbor. In particular, using the maximum principle, we can conclude that at equilibrium the maximal confidence is attained for the influencers.

Remark 9.7. (Distorsion of the Poincaré metric)

We based the previous analysis on the standard (conformal) Poincaré metric whereas the Fisher metric (38) natively comes with diagonal coefficients 1 and 2. More generally, one may consider the 1-dimensional family of metrics associated to a new parameter $\kappa \in(0,+\infty)$ :

$$
\left\langle I_{\kappa} d \theta \mid d \theta\right\rangle=\frac{1}{\sigma^{2}} d m^{2}+\frac{\kappa}{\sigma^{2}} d \sigma^{2}
$$

This factor $\kappa$ does not change anything in the previous mathematical developments, except that geodesics are no longer circles but ellipses: specifically, the pullback of the metric $I_{\kappa}$ by the diffeomorphism $(m, \sigma) \mapsto(m, \sigma / \sqrt{\kappa})$ of $\mathbb{H}^{2}$ is nothing else than $\kappa I_{1}$. It rather affects the model itself. With the choice $\kappa=1$ that was made, consider two convinced influencers who disagree (mean opinions $m_{0}<m_{1}$, and $\sigma_{0}=\sigma_{1}$ small compared to $m_{1}-m_{0}$ ), and a third agent equally influenced by both. In $\mathbb{H}^{2}$, it corresponds to points almost touching the $m$ axis, and the opinion of the third one lies in the middle of the half circle meeting perpendicularly the $m$ axis, and containing the two points. As a consequence, the standard deviation of the influenced agent is about half the difference between the mean opinion of influencers, which is not unrealistic in terms of modeling. It means in particular that the influenced agent gives a $68 \%$ chance that the value lies between those of influencers, and $32 \%$ chance that it is above the largest one, or below the smallest one. For the Fisher metric $(\kappa=2)$, the geodesic is supported by a half ellipse of excentricity $1 / \sqrt{2}$, so that the standard deviation of the third agent is now $m_{1}-m_{0}$ divided by $2 \sqrt{2}$. The influenced agent now gives $85 \%$ chance that the value lies between those of influencers. More generally, $\kappa$ can be used as a parameter to model the behavior of agents. A large $\kappa$ makes ellipses closer to (horizontal) straight segments, meaning that the third agent is more and more convinced that the value is close to the mean between those of influencers. For very large $\kappa$ 's, we recover an unrealistic behavior similar to the one of Wasserstein interpolation. More interestingly, small values of $\kappa$ increase the standard deviation of the third agent. It can be used to implement the following behavior: if an agent is influenced by two influencers so far away in the $\kappa$-Fisher metric that the coexistence of their contradictory and mutually discrediting opinions does not make sense (at the limit the support of probability measures have an empty intersection), then this agent simply disregards influencers, which is implemented by the fact that this standard deviation of the influenced agent is much larger than $m_{1}-m_{0}$, which in some way kills the influence power of both influencers. Note that, by pre-negociating, those influencers may conciliate their means, and become much more influential upon the third agent by cheating on the opinion which they display. 
Remark 9.8. (Parametric character of the approach)

To obtain the Fisher metric, we estimated the costs associated to infinitesimal variations of densities upon relative entropy (or Kullback-Leibler divergence, see (35)). We then restricted ourselves to Gaussian densities, which led us to the (distorted) Poincaré half-plane $\mathbb{H}^{2}$. One may wonder whether it would be possible and relevant to extend this approach to more general classes of densities, up to possibly non-parametric families. If one aims at keeping relevant interpolation properties, the answer is negative. Indeed, beyond the difficulty to properly define and compute geodesics in a general setting (see for instance [6]), it should be noted that the rigidity of the Gaussian family plays an essential role in the overall approach. In a non-parametric context, the relative entropy is fully disconnected from the underlying geometry, i.e. the geometry of the space in which the opinion $u$ lies. This fact is made clear by the following consideration: one may apply this approach to discrete probabilities defined on a discretization of the space $\Omega$ (to which $u$ belongs). The density would then be described by a vector of probabilities $p_{1}, \ldots, p_{m} \geq 0$, with $\sum p_{i}=1$. Formula (35) defines a Riemannian metric on the standard simplex (see e.g. 10]), but it can be checked that this metric is invariant by any permutation of indices. In particular, if one interpolates according to this metric between $\delta_{1}=(1,0, \ldots, 0)$ and $\delta_{m}=(0, \ldots, 0,1)$, one straightforwardly obtains that the whole geodesic does not charge intermediate indices, $2, \ldots, m-1$ : the approach does not interpolate in the opinion state in a manner which makes sense from the modeling standpoint, it rather performs some sort of teleportation, like the linear interpolation would do. Forcing densities to a have a given unimodal shape (like Gaussian) is essential to force the geodesic to go through intermediate values of opinion between endpoints.

\section{BibliographicAl NOTES}

Before presenting some bibliographical insights about opinion propagation, we would like to emphasize, as recalled in the introduction, that our goal was rather to connect this topic to other well studied mathematical objects. A long list of reference would be required for each of the mathematical objects mentioned in these notes if we were aiming exhaustivity. However, as it is not our main purpose, and as we hope that our presentation is self-contained enough, we shall be very brief. Most of the material in the first sections is usually covered in undergraduate years in link with linear algebra. For the study of Markov chains evoked in Section 2 and 5 , we refer for instance to the first four chapters of [56], and 18] for a more thorough presentation. As for the adjoint method which we presented in some details in Section 3. we refer the reader to [51] for a detailed presentation of this strategy to compute the gradient of various functionals. Concerning the discretization of PDE in Section 6. we invite the reader to take a look at, for instance [41. Sections 7, 8 and the model of "fuzzy" opinion in Section 9 are original developpements that we investigated during the writing of these notes. Nevertheless, for the topic of optimal transport mentioned in the beginning of Section 9, one can find an introduction at a graduate level in 57.

Opinion propagation, understood in a wide sense, is a very large topic: although we have examined it with a mathematical point of view, questions of epistemology (how do people form opinions and beliefs ?) and sociology (how do people change their belief when interacting with others?) are fully relevant to such a study. In this section, we will stick to the mathematical literature on the topic, trying to explain the different models that have already been proposed.

We start by mentioning two recent articles by Proskurnikov and Tempo [53,54] which are not only a rather exhaustive survey of the different models of opinion propagation proposed in the last decades, but are also written in a accessible way.

A first appearance of the linear evolution model 22] can be found in the work of French [30. The main goal of this article is the justification of the model, as well as the exhibition of sufficient conditions for the distribution of opinions to evolve toward a consensus. The same model is considered in the paper by DeGroot [24], with the help of the language of Markov chains to study the convergence to a consensus. Moreover, DeGroot describes the opinion not by a single variable, but by a distribution of probability called opinion pool evolving according 
to 33 . We also refer to 64 for a modeling approach which accounts for the fuzzy character of both opinion and its way to propagate.

We emphasize, as mentioned in 65, that this linear model differs from a previous approach developed in the 1940s and 1950s, where one considers that the information flows directly from the media to opinion leaders and then to the public: the idea of the linear model is to take in account the fact that information flows in every direction, and that direct influence in itself is not enough to understand the dynamic of opinion formation.

Several improvements have been made to the original linear model. For instance, Friedkin and Johnsen 32 considered that people keep in mind a tiny fraction of their initial opinion. Note that this feature can be straightforwardly incorporated to the linear model introduced in Section 1 by adding one "personal influencer" per agent, and by considering that this personal influencer keeps over the evolution process the initial opinion, and thereby continues to attract their target toward this initial opinion. This is to be put in contrast with the persuasion bias 26]: in the model proposed by French and DeGroot (and investigated in these notes), agents have no memory in the sense that, at each iteration, they behave like they are receiving information from their neighbors for the first time. As pointed out by [26, this effect pushes toward consensus and also leads multi-dimensional opinions to polarize along a uni-dimensional axis. People have complexified the linear model by making the influence coefficients $K$ depend on both time and the value of the opinions. An example is given by Hegselmann and Krause [35] where they introduce the bounded confidence model: an agent do not listen to their neighbors if their neighbor's opinion is too far away (specifically: above a given threshold) from theirs. This effect (which can be incorporated in the non-linear model of Section 7) leads to a fragmentation of the distribution of opinions at equilibrium. The Hegselmann and Krause article has started a large line of research leading to many variations from the original model, see for instance the survey by Lorenz [42].

Some models have proposed to relax the non-negativity assumption of the coefficients $\left(K_{x y}\right)_{x y}$ quantifying the inter-individual influences: this leads to antagonistic interactions, where an agent might want their opinion to differ from the one of their neighbor. The maximum principle is no longer valid hence almost all of the analysis of the present lecture notes does not apply. Nevertheless as understood in [3], depending on the signs of the inter-individual influences, the distribution of opinions can converge to a "agreed upon dissensus": that is, a stable situation where only two opposite opinions exist. Such kind of result has been extended to very general non-linear interactions [4] and to the case where the graph structure changes with time [55].

We mention a recent line of research, where one assumes that there is a large number of agents, and looks only at the statistical distribution of opinions of the agents. If the agents have an opinion about a parameter $u \in \Omega$, the unknown is a distribution $f(t, u)$ denote the density of agents having the opinion $u$ at time $t$. The evolution of this distribution of opinions is written with PDEs similar to the Boltzmann equation, in the spirit of kinetic theory 15,61 .

In these notes, we have considered that the network $(V, K)$, describing the interpersonal influences, is exogenous: it is a data of the problem and it does not evolve. Several questions arise, as: what do real social networks look like? What are the typical values for the ingoing and outgoing degrees, the diameters? Could one generate random networks that look like real ones? For all of these questions, we refer to the survey by Newman 47 . and references therein. A striking characteristic of real social networks is the "small world effect", namely that any two vertices are connected by a path of short length (no more than 5 or 6 ), independently from the size of the network. Even if the network is known, but potentially very large, retrieving its statistical features or its influencers could be a challenge [12,37,40].

Related to the end of the last paragraph is the confrontation with experimental data. Experiments in a controlled environment, with a small number of subjects, has been performed for instance by Friedkin and Johnsen 31. The emergence of (digital) social network led to the possibility of studying propagation of opinion on large datasets, see for instance the recent article 34] which analyze how investors react on twitter after announcements of companies' earnings.

Eventually, we mention that there are other ways to model people's belief than with just a single value. Bayesian theory aims at describing people's belief and how they evolve given new evidences. Applications of this theory to opinion propagation can be found in 11. At a broader scale, other than just opinion or beliefs, some 
other interactions could take place on a network: epidemic propagation [49], game theory [9] or propagation of defaults in financial networks [8]. We refer the reader to the book of Easley and Kleinberg [29] for an exhaustive account of social phenomena on networks.

\section{APPENDIX}

\section{A. Propagation on graphs of the mapping type}

We consider here the situation where the influence graph $(V, E)$ identifies to a mapping $\Phi: V \rightarrow V$, i.e. for any $x$, one has $K_{x y}=1$ for a single $y=\Phi(x)$. In other words

$$
K_{x y}=\delta_{\Phi(x), y},
$$

where $\delta$ is the Kronecker delta. In this situation, the discrete evolution problem writes

$$
u_{x}^{k+1}=u_{\Phi(x)}^{k} \quad \forall x \in V
$$

so that

$$
u^{k}=u^{k-1} \circ \Phi=u^{k-2} \circ \Phi \circ \Phi=\cdots=u^{0} \circ \Phi^{k},
$$

which is the pullback of $u^{0}$ by $\Phi^{k}=\Phi \circ \cdots \circ \Phi$.

One-to-one and onto graph

We consider here the bijective case : for any $y, K_{x y}=1$ for a single $x$, which expresses that $\Phi$ is one-to-one and onto.

Proposition A.1. In the case when $\left(K_{x y}\right)$ encodes a bijection (one-to-one correspondence), the sequence $\left(u^{k}\right)$ obtained from 22 is periodic for any initial state $u^{0}$.

Proof. This is a direct consequence of the fact that any permutation can be decomposed as the product of cycles with disjoint supports. Indeed, consider, for any $x \in V$, the sequence $\left(\Phi^{k}(x)\right)$, with

$$
\Phi^{k}(x)=\underbrace{\Phi \circ \cdots \circ \Phi}_{k \text { times }}(x) .
$$

Since $V$ is finite, there is a $L \geq 1$ such that $\left(\Phi^{L}(x)\right)$ is equal to $\left(\Phi^{j}(x)\right)$, for some $j<L$. Let us consider the smallest of those $L^{\prime} s$. If the associated $j$ is not 0 , then $\Phi\left(\Phi^{L-1}(x)\right)=\Phi\left(\Phi^{j-1}(x)\right.$ ), which implies (from the one-to-one character of $\Phi)$ that $\left.\Phi^{L-1}(x)\right)=\Phi^{j-1}(x)$. It contradicts the minimal character of $L$. Therefore $j=0$, and the sequence $\left(\Phi^{k}(x)\right)$ is $L$-periodic. For any $x^{\prime}$ in the orbit of $x$, i.e. equal to one of the $\left(\Phi^{k}(x)\right)$ 's, the sequence is also $L$-periodic, and the restriction of $\Phi$ to the orbit of $x$ is a cycle. This orbit and its complementary set are stable by $\Phi$, and the same approach can be applied to the complementary set. Since the set is finite, it leads to decomposing the set $V$ onto disjoint stable subsets, in which $\Phi$ is a cycle. The solution to (2) is therefore periodic, with a period at most equal to the least common multiple of the cycles' orders.

The l.c.m. (Least Common Multiple) of the cycles' orders is the order of $\Phi$ in the symmetric group, i.e. the smallest integer $m$ such that $\Phi^{m}=\mathrm{I}_{\mathrm{d}}$. The actual period of the solution to $(2)$ may be smaller that $m$, e.g. by considering a uniform initial state $u^{0}$. In the generic case where the values of $u^{0}$ are pair-wise distinct (at least within each orbit), the period is equal to $m$. 


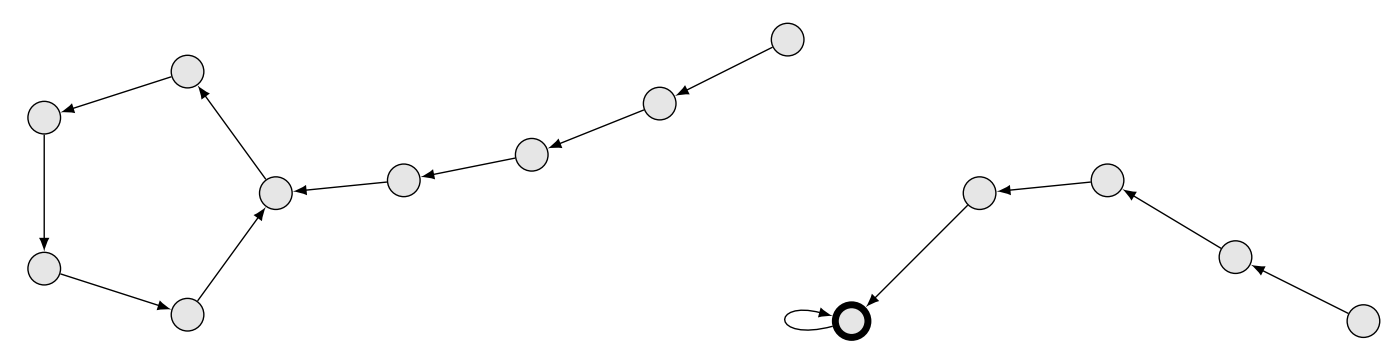

FiguRE 11. Lollypop graph, only one influencer (circled node).

Remark A.2. (Landau Function)

A natural question arises : for a given cardinal $N$ of $V$, what is the maximal period for the solution to Model (2) ? Let us first notice that this period obviously depends on the initial state. To fix the ideas, we shall consider that all opinions are initially pairwise distinct. The period issue is related to the so-called Landau function $N \longmapsto g(N)$, which is the maximal order of an element of the symmetric group $S_{N}$. This function $g(\cdot)$ is obviously non-decreasing, with $g(1)=1, g(2)=2, g(3)=3, g(4)=4, g(5)=6, \ldots$ More generally, for any $M \in \mathbb{N}$, with the following factorization into primes

$$
M=p_{1}^{\alpha_{1}} p_{2}^{\alpha_{2}} \ldots p_{k}^{\alpha_{k}},
$$

let us introduce by $\ell(M)=p_{1}^{\alpha_{1}}+p_{2}^{\alpha_{2}}+\cdots+p_{k}^{\alpha_{k}}$. It was proved in 1953 (see [38]) that

$$
g(N)=\max _{\ell(M) \leq N} M
$$

which implies in particular $\ell(g(N)) \leq N$. We refer to 25] for an historical account of the advances made on this Landau Function, together with the description of an efficient algorithm to compute its value for large $N$ 's.

\section{Graph of the mapping type}

Let us now assume that the index of each vertex is equal to 1 , i.e. for any $x, K_{x y}=1$ for some $y \in V$. Like in the bijective situation, it is fruitful to consider, for any $x \in V$, the sequence $\left(\Phi^{k}(x)\right)$. As previously, since $V$ is finite, there is a $L \geq 1$ such that $\left(\Phi^{L}(x)\right)$ is equal to $\left(\Phi^{j}(x)\right)$, for some $j \leq L$. If $j=0$ like in the bijective case, one recovers a $j$-cycle. If $j=L$, then necessarily the corresponding $y=\Phi^{L}(x) \in \Gamma$, and the orbit is a straight path to $\Gamma$. If $0<j<L$, then the path connects $x$ to a cycle of length $L-j+1$. Any solution to (2) is then periodic after some time, with a period at most equal to the l.c.m. of the periods $L-j+1$ exhibited previously. Figure 11 presents a graph with two connected components, with two different types of attractors : a cycle (left) and a single vertex (right). In this situation, the period is 6 , and number of step after which it is periodic is 4 . Note that all the initial opinions lying on the linear parts of the graph have been washed out in the evolution process.

\section{B. Effective computations of BARYCEnters for the Fisher Metric}

In this section, we would like to explain how one can in practice compute barycenters in the Poincaré half plan£. This appendix will be less detailed than the rest of these notes: even though we have tried to be as clear as possible, the good framework to understand what we are doing is statistics on Riemannian manifolds, which is out of the scope of the present work. For a good introduction to the topic, we refer to 50].

\footnotetext{
${ }^{9}$ The final implementation is available at https://www.esaim-proc.org/10.1051/proc/202067016/olm
} 
The problem we are interested in is the following. Assume we have Gaussian densities $\rho_{1}, \rho_{2}, \ldots, \rho_{n}$ over the real lines, with means $m_{1}, m_{2}, \ldots, m_{n}$ and standard deviations $\sigma_{1}, \sigma_{2}, \ldots, \sigma_{n}$. A couple $\theta=(m, \sigma)$ will be though as a point in the Poincaré half plane

$$
\mathbb{H}^{2}=\mathbb{R} \times(0,+\infty)
$$

This space $\mathbb{H}^{2}$ is endowed with the distance $d_{F}$ which corresponds to Fisher distances between Gaussian distributions (up to a rescaling of the $\sigma$-axis) as described above in Section 9 Assuming that we have positive weights $K_{1}, K_{2}, \ldots, K_{n}$, and points $\theta_{1}, \theta_{2}, \ldots, \theta_{n}$ we want to find $\theta \in \mathbb{H}^{2}$ which minimizes

$$
E(\theta)=\sum_{i=1}^{n} \frac{K_{i}}{2} d_{F}\left(\theta, \theta_{i}\right)^{2}
$$

as such an optimal $\theta$ is (by definition) the barycenter of the $\theta_{i}$ with weights $K_{i}$. As the space $\mathbb{H}^{2}$ is finitedimensional (of dimension 2), a gradient descent algorithm is appropriate. However, to get faster results and guarantees of convergence, it is more relevant to a do a gradient descent in the geometry of $\mathbb{H}^{2}$ induced by $d_{F}$, and not in the naive Euclidean geometry.

We recall here some notions of Riemannian geometry as well as some explicit formulas in the Poincaré half plane. First, even though we will not make a great use of it, we recall that the metric tensor of $\mathbb{H}^{2}$ at a point $\theta=(m, \sigma)$ is

$$
\left\langle I_{\theta}(d m, d \sigma) \mid(d m, d \sigma)\right\rangle=\frac{(d m)^{2}+(d \sigma)^{2}}{\sigma^{2}} .
$$

Moreover, as explained above, $d_{F}$ is the Riemannian distance associated to $\left(\mathbb{H}^{2}, F\right)$. It can be expressed as

$$
d_{F}\left(\theta_{0}, \theta_{1}\right)=\operatorname{acosh}\left(1+\frac{\left(m_{0}-m_{1}\right)^{2}+\left(\sigma_{0}-\sigma_{1}\right)^{2}}{\sigma_{0} \sigma_{1}}\right) .
$$

Definition B.1. Let $I$ an interval of $\mathbb{R}$ and $c>0$. A curve $t \in I \mapsto \theta(t)=(m(t), \sigma(t)) \in \mathbb{H}^{2}$ is called a geodesic traveled at speed $c$ if and only if, for any $s<t$ elements of $I$,

$$
d_{F}(\theta(s), \theta(t))=c|t-s| .
$$

Not only does it mean that the curve is traveled at constant speed, but it also says that in fact the curve is the fastest way to go from $\theta(s)$ to $\theta(t)$ if $s<t$. To make the link with the metric tensor, we know that for a geodesic $\theta$ traveled at speed $c$, the latter can be retrieved by

$$
c^{2}=\left\langle I_{\theta(t)} \dot{\theta}(t) \mid \dot{\theta}(t)\right\rangle .
$$

where $\dot{\theta}(t) \in \mathbb{R}^{2}$ denotes the usual time-derivative of $\theta$ at time $t$. In the space $\mathbb{H}^{2}$, the geodesics are fully classified. We will not prove this result, there are many elegant ways to deduce it and we refer for instance to 21 .

Proposition B.2. For any $A, \theta_{0}, c \in \mathbb{R}$ and $B>0$, the curve

$$
t \mapsto\left(\begin{array}{c}
A+B \tanh \left(c\left(t-t_{0}\right)\right) \\
B \cosh ^{-1}\left(c\left(t-t_{0}\right)\right)
\end{array}\right)
$$

defined on $\mathbb{R}$ is a geodesic traveled at constant speed $|c|$. Moreover,

$$
t \mapsto\left(\begin{array}{c}
A \\
e^{c\left(t-t_{0}\right)}
\end{array}\right)
$$


is also a geodesic defined on $\mathbb{R}$ traveled at constant speed $|c|$.

Furthermore, any geodesic traveled at constant speed coincides with the ones defined in (44) or (45) for suitable $A, B, c$ and $t_{0}$.

We emphasize that $\cosh ^{-1}: \mathbb{R} \rightarrow(0,1]$ denotes $1 / \cosh$ and not the reciprocal map of cosh.

Remark B.3. Recalling the identity $\cosh ^{2}-\sinh ^{2}=1$, one can check that, geometrically, the geodesics described by (44) are circles centered at $(A, 0)$ and of radius $B$. On the other hand, the ones described by (45) are half-lines issued from $(A, 0)$ and aligned with the $\sigma$-axis. Some geodesics are depicted in Figure 9 The parameter $t_{0}$ simply amounts for temporal translation. Eventually, as the geodesics can be traveled in two different directions, we allow $c$ to take negative values, and the speed of the geodesic is given by $|c|$.

With these geometric considerations, we hope that the reader is convinced that the following result indeed holds.

Proposition B.4. If $\theta_{0}$ and $\theta_{1}$ are two points in $\mathbb{H}^{2}$, then there exists a unique geodesic $t \in[0,1] \mapsto \theta(t)$ traveled at constant speed such that $\theta(0)=\theta_{0}$ and $\theta(1)=\theta_{1}$, and this geodesic is traveled at speed $d_{F}\left(\theta_{0}, \theta_{1}\right)$.

Remark B.5. Actually, it is rather easy to get the expression of such a geodesic. If $m_{0}=m_{1}$, we know that it must lie on the line $\left\{m=m_{0}\right\}$, i.e. we take $A=m_{0}$ in 45 . Then we conclude that we must take $c=\ln \left(\sigma_{1} / \sigma_{0}\right)$ and $t_{0}=-\ln \left(\sigma_{0}\right) / c$.

On the other hand, if $m_{0} \neq m_{1}$, we know that we must take a geodesic of the form (44). To compute $A$, we just know that $(A, 0)$ must be equidistant for the Euclidean distance from $\left(m_{0}, \sigma_{0}\right)$ and $\left(m_{1}, \sigma_{1}\right)$. It leads to the expression

$$
A=\frac{m_{0}+m_{1}}{2}+\frac{\sigma_{1}^{2}-\sigma_{0}^{2}}{2\left(m_{1}-m_{0}\right)} .
$$

Once $A$ is known, we define $B$ as the Euclidean distance between $(A, 0)$ and $\theta_{0}$, i.e. $B=\sqrt{\left(A-m_{0}\right)^{2}+\sigma_{0}^{2}}$. If we are in the case $m_{0}<m_{1}$ (the reverse case is symmetric), we know that $c>0$, moreover $|c|=d_{F}\left(\theta_{0}, \theta_{1}\right)$. Eventually, we can recover $t_{0}$ by

$$
t_{0}=\frac{1}{c} \operatorname{atanh}\left(\frac{A-m_{0}}{B}\right)
$$

Definition B.6. Let $\theta_{0}$ and $\theta_{1}$ two points in $\mathbb{H}^{2}$. The logarithm of $\theta_{1}$ with respect to $\theta_{0}$, denoted by $\log _{\theta_{0}}\left(\theta_{1}\right)$ is defined as

$$
\left(\left.\frac{d m(t)}{d t}\right|_{t=0},\left.\frac{d \sigma(t)}{d t}\right|_{t=0}\right) \in \mathbb{R}^{2}
$$

where $t \in[0,1] \mapsto \theta(t)=(m(t), \sigma(t))$ is the unique geodesic traveled at constant-speed between $\theta_{0}$ and $\theta_{1}$.

The temporal time-derivative taken is the usual one, where $\mathbb{H}^{2}$ is endowed with its Euclidean structure. Hence, $\log _{\theta_{0}}\left(\theta_{1}\right)$ is nothing else than a vector in $\mathbb{R}^{2}$, in fact thought as an element of the tangent space of $\mathbb{H}^{2}$ at $\theta_{0}$.

Remark B.7. As pointed by Remark B.5, the computation of the constant-speed geodesics between two given points of $\mathbb{H}^{2}$ can be done in closed form, thus so does $\log _{\theta_{0}}\left(\theta_{1}\right)$.

In short, $\log _{\theta_{0}}\left(\theta_{1}\right)$ is just the initial speed of the geodesic joining the two points $\theta_{0}$ and $\theta_{1}$. Conversely, if one is given a point $\theta_{0} \in \mathbb{H}^{2}$ and a vector $(\dot{m}, \dot{\sigma}) \in \mathbb{R}^{2}$, there exists a unique constant-speed geodesic defined on $\mathbb{R}$ whose position at time $t=0$ is $\theta_{0}$ and whose speed at time $t=0$ is $(\dot{m}, \dot{\sigma})$. It tells us that the map $\theta_{1} \mapsto \log _{\theta_{0}}\left(\theta_{1}\right)$ is in fact one to one.

Definition B.8. Let $\theta_{0} \in \mathbb{H}^{2}$ be fixed. We define the exponential map from $\theta_{0}$ as

$$
\exp _{\theta_{0}}: \mathbb{R}^{2} \rightarrow \mathbb{H}^{2}
$$

as the inverse of $\log _{\theta_{0}}(\cdot): \mathbb{H}^{2} \rightarrow \mathbb{R}^{2}$. 
Remark B.9. Similarly to the logarithm map, the exponential map can be computed in closed form. Indeed, let us assume that we have a point $\theta_{0}=\left(m_{0}, \sigma_{0}\right)$ and a vector $(\dot{m}, \dot{\sigma})$. We want to compute $\exp _{\theta_{0}}((\dot{m}, \dot{\sigma}))$. To that end, we need to identify the constant-speed geodesic passing at $\left(m_{0}, \sigma_{0}\right)$ with velocity $(\dot{m}, \dot{\sigma})$. Given 443 , we know that $|c|$ the speed of the curve will be

$$
|c|=\sqrt{\left\langle I_{\theta_{0}}(\dot{m}, \dot{\sigma}) \mid(\dot{m}, \dot{\sigma})\right\rangle}=\frac{\sqrt{\dot{m}^{2}+\dot{\sigma}^{2}}}{\sigma_{0}} .
$$

Now, if $\dot{m}=0$, the geodesic is a vertical line, i.e. we have to take $A=m_{0}$. Assuming that $\dot{\sigma}>0$, which means $c>0$, we just have to take $t_{0}=-\ln \left(\sigma_{0}\right) / c$ and evaluate Formula (45) at $t=1$.

On the other hand, if $\dot{m} \neq 0$, we have to figure on which circle the geodesic is located. The center of this circle lies on the axis $\sigma=0$, while it passes through $\theta_{0}$ and its radius is orthogonal to $(\dot{m}, \dot{\sigma})$. It is not difficult to conclude that the center is $(A, 0)$ with

$$
A=m_{0}+\frac{\sigma_{0} \dot{\sigma}}{\dot{m}} .
$$

Once we have $A$, we have to take $B$ as the Euclidean distance between $(A, 0)$ and $\theta_{0}$, i.e. $B=\sqrt{\left(A-m_{0}\right)^{2}+\sigma_{0}^{2}}$. Then, assuming that $\dot{m}>0$ (the reverse situation is symmetric), we know that $c>0$ and we can use (46) to compute $t_{0}$. Then we use (44) and evaluate it at time $t=1$.

The exponential and logarithm maps could be defined in fact in any Riemannian manifold (though they might not be globally defined as it is the case here), and are the building block to do statistics in this framework.

As a first result, as $\mathbb{H}^{2}$ is in fact a Hadamard manifold, we can apply [50, Corollary 2] to get the following.

Proposition B.10. Let $\theta_{1}, \theta_{2}, \ldots, \theta_{n}$ be points in $\mathbb{H}^{2}$ and $K_{1}, K_{2}, \ldots K_{n}$ be positive weights. Then there exists a unique $\theta \in \mathbb{H}^{2}$ barycenter of the $\theta_{i}$ 's with weights $K_{i}$ 's (i.e. a global minimizer of the functional $E$ defined in (42)), and it is characterized by

$$
\sum_{i=1}^{n} K_{i} \log _{\theta}\left(\theta_{i}\right)=0
$$

Notice that the optimality condition is in fact an equality of vectors in $\mathbb{R}^{2}$ (thought as the tangent space to $\mathbb{H}^{2}$ at $\theta$ ). Actually, the left hand side of (47) is nothing else (up to a minus sign) than the gradient, with respect to the Fisher scalar product $I_{\theta}$ of the functional $E$ at the point $\theta$.

Moreover, we deduce a gradient descent to compute an approximation of the barycenter. For simplicity, we assume that the weights are normalized in such a way that $\sum K_{i}=1$. The algorithm reads

(1) Take $\theta^{0}$ an initial guess for the barycenter (e.g. one of the $\theta_{i}, i=1,2, \ldots, n$ ).

(2) If $\theta^{k}$ is known, compute $\nabla_{F} E\left(\theta^{k}\right)=-\sum_{i=1}^{n} K_{i} \log _{\theta^{k}}\left(\theta_{i}\right) \in \mathbb{R}^{2}$.

(3) If $\nabla_{F} E\left(\theta^{k}\right)$ is smaller than a prescribed tolerance, exit the algorithm and take $\theta^{k}$ as the barycenter.

(4) Else, compute $\theta^{k+1} \in \mathbb{H}^{2}$ via

$$
\theta^{k+1}=\exp _{\theta^{k}}\left(-\tau^{k} \nabla_{F} E\left(\theta^{k}\right)\right)
$$

where $\tau^{k}>0$ is a time-step and go back to point (2).

The crucial difference, with a classical gradient descent algorithm, is that in the update step (48) we use the exponential map: with this choice we are sure to stay in the manifold $\mathbb{H}^{2}$, and as the gradient was computed for the Fisher geometry it makes sense to move along geodesics in the Fisher distance. Actually, in the Fisher geometry, the Hessian of $E$ is equal to the identity matrix, hence the gradient descent can in fact be interpreted as Newton's algorithm.

The time-step can be chosen either constant, or using a backtracking line search as for the usual gradient descent. With some appropriate choices, convergence to the uniqe barycenter can be guaranteed, see for instance 13 . 
Notice, as explained in Remarks B.7 and B.9, that this algorithm can be implemented in practice on $\mathbb{H}^{2}$ as there are explicit formulas for the logarithm and exponential maps.

\section{REFERENCES}

[1] D. Acemoglu, A. Ozdaglar, Opinion dynamics and learning in social networks. Dynamic Games and Applications, 2011, vol. 1, no 1, pp. 3-49.

[2] M. Agueh, G. Carlier, Barycenters in the Wasserstein space. SIAM Journal on Mathematical Analysis 43.2 (2011): 904-924.

[3] C. Altafin, Consensus problems on networks with antagonistic interactions. IEEE Transactions on Automatic Control 58.4 (2012): 935-946.

[4] C. Altafini, Dynamics of opinion forming in structurally balanced social networks. PloS one 7.6 (2012): e38135.

[5] F. Al Reda, B. Maury, Interpretation of Finite Volume discretization schemes for the Fokker Planck equation as gradient flows for the discrete Wasserstein distance, Topological Optimization and Optimal Transport in the Applied Sciences 15, 333 (2017).

[6] S.-I. Amari, H. Nagaoka, Methods of information geometry, American Mathematical Soc., 2007.

[7] W. F. Ames, Numerical methods for partial differential equations, Academic press, 2014.

[8] H. Amini, R. Cont, and A. Minca, Resilience to contagion in financial networks, Mathematical finance, 2016, vol. 26, no 2, pp. 329-365.

[9] K. R. Apt, S. Simon, and D. Wojtczak, Coordination games on directed graphs, Electronic Proceedings in Theoretical Computer Science 215, pp. 67-80, arXiv preprint arXiv:1606.07517, 2016.

[10] N. Ay, J. Jost, H. V. Lê, and L.L. Schwachhöfer, Finite Information Geometry, in: Information Geometry. Ergebnisse der Mathematik und ihrer Grenzgebiete. 3. Folge / A Series of Modern Surveys in Mathematics, vol 64. Springer, Cham.

[11] J.-D. Benamou, Y. Brenier, A computational fluid mechanics solution to the Monge-Kantorovich mass transfer problem, Numer. Math. 84 (2000), no. 3, 375-393.

[12] A. Ben-Hamou, R. I. Oliveira, and Y. Peres, Estimating graph parameters with random walks, arXiv preprint arXiv:1709.00869, 2017.

[13] G. de Carvalho Bento, S. D. Barbosa Bitar, J. X. da Cruz Neto, P. R. Oliveira, J. C. de Oliveira Souza, Computing Riemannian Center of Mass on Hadamard Manifolds, Journal of Optimization Theory and Applications 183 (3), 977-992.

[14] F. Bernicot, B. Maury, and D. Salort, A 2-adic approach of the human respiratory tree, Netw. Heterog. Media 5 (2010), no. 3 , 405-422.

[15] L. Boudin, F. Salvarani, The quasi-invariant limit for a kinetic model of sociological collective behavior. Kinet. Relat. Models, 2(3):433-449, 2009.

[16] C. Bordenave, P. Caputo, and D. Chafaï, Circular Law Theorem for Random Markow Matrices, Probability Theory and Related Fields 152, 3-4 (2012) 751-779.

[17] K. C. Border, Fixed point theorems with applications to economics and game theory, Cambridge university press, 1985.

[18] P. Brémaud, Markov chains: Gibbs fields, Monte Carlo simulation, and queues. Vol. 31. Springer Science \& Business Media, 1999.

[19] H. Brezis, Opérateurs Maximaux Monotones et Semi-groupes de contractions dans les espaces de Hilbert, AM, North Holland, 1973.

[20] M. R. Bridson, A. Haefliger, Metric spaces of non-positive curvature. Vol. 319. Springer Science \& Business Media, 2013.

[21] J. W. Cannon, W. J. Floyd, R. Kenyon, and W. R. Parry (1997). Hyperbolic geometry. Flavors of geometry, 31, 59-115.

[22] T. H Cormen, C.E. Leiserson, R. Rivest, C. Stein, Introduction to algorithms, MIT press Cambridge, 2009.

[23] S. I. R. Costa, S. A. Santos, J. E. Strapasson, Fisher information distance: A geometrical reading, Discrete Applied Mathematics Volume 197, 31 December 2015, pp. 59-69. https://arxiv.org/pdf/1210.2354.pdf

[24] M. H. Degroot, Reaching a Consensus, Journal of the American Statistical Association Volume 69, 1974 - Issue 345.

[25] M. Deléglise, J.-L. Nicolas, P. Zimmermann, Landau's function for one million billions, Journal de théorie des nombres de Bordeaux, Tome 20 (2008) no. 3, pp. 625-671 https://hal.archives-ouvertes.fr/hal-00264057/

[26] P. M. Demarzo, D. Vayanos, and J. Zwiebel, Persuasion bias, social influence, and unidimensional opinions. The Quarterly journal of economics, 2003, vol. 118, no 3, pp. 909-968.

[27] J. L. Doob, Classical Potential Theory and Its Probabilistic Counterpart, Springer-Verlag Berlin Heidelberg, 2001.

[28] P. Doyle, J. Snell, Random Walks and Electric Networks, Mathematical Association of America (1984).

[29] D. Easley, J. Kleinberg, Networks, crowds, and markets, Cambridge, Cambridge University Press, 2010.

[30] J. R. Jr. French, A formal theory of social power, Psychological review, 1956, vol. 63, no 3, pp. 181.

[31] N. E. Friedkin, E. C. Johnsen, Social influence and opinions, Journal of Mathematical Sociology, 1990, vol. 15, no 3-4, pp. 193-206.

[32] N. E. Friedkin, E. C. Johnsen, Social positions in influence networks, Social Networks, 1997, vol. 19, no 3, pp. $209-222$.

[33] J. Ghaderi , R. Srikant, Opinion Dynamics in Social Networks with Stubborn Agents: Equilibrium and Convergence Rate, Automatica, vol. 50, no. 12, 2014. 
[34] R. Giannini, P. Irvine, and T. Shu, The convergence and divergence of investors' opinions around earnings news: Evidence from a social network, Journal of Financial Markets, 2019, vol. 42, pp. 94-120.

[35] R. Hegselmann, U. Krause, Opinion dynamics and bounded confidence: models, analysis and simulation, Journal of Artificial Societies and Social Simulation vol. 5, no. 3, 2002.

[36] P. Henrici, Bounds for iterates, inverses, spectral variation and fields of values of non-normal matrices, Numerische Mathematik 4, pp. 24-40 (1962)

[37] F. Iannelli, M. S. Mariani, and I. M. Sokolov, Influencers identification in complex networks through reaction-diffusion dynamics Physical Review E, 2018, vol. 98, no 6, 98:062302.

[38] E. Landau, Über die Maximalordnung der Permutationen gegebenen Grades, Archiv. der Math. und Phys., Sér. 3, 5 (1903), 92-103, Handbuch der Lehre von der Verteilung der Primzahlen I, 2nd ed, Chelsea, New-York, 1953, $222-229$.

[39] Y. LeCun, Y. Bengio, G. Hinton, Deep learning, Nature volume 521, pages 436-444.

[40] J. Leskovec and C. Faloutsos, Sampling from large graphs. Proceedings of the 12th ACM SIGKDD international conference on Knowledge discovery and data mining, ACM, 2006. pp. 631-636.

[41] R. LeVeque, Finite difference methods for ordinary and partial differential equations: steady-state and time-dependent problems, Vol. 98. Siam, 2007.

[42] J. Lorenz, Continuous opinion dynamics under bounded confidence: A survey, International Journal of Modern Physics C 18.12 (2007): 1819-1838.

[43] J. Maas, Gradient flows of the entropy for finite Markov chains, Journal of Functional Analysis, Volume 261, Issue 8, 15 October 2011, Pages 2250-2292.

[44] B. Maury, S. Faure, Crowds in Equations. London: World Scientific Publishing Europe Ltd (2018).

[45] B. Maury, D. Salort, C. Vannier, Trace theorems for trees, application to the human lung, Network and Heterogeneous Media, Volume 4, Number 3, September 2009 pp. 469-500

[46] R. J. McCann, A Convexity Principle for Interacting Gases, Advances in Mathematics 128, 153-179 (1997).

[47] M. E. J. Newman, The structure and function of complex networks, SIAM review, 2003, vol. 45, no 2, p. $167-256$.

[48] S.-I. Ohta, Advances in Geometry, Barycenters in Alexandrov spaces of curvature bounded below, Volume 14, Issue 4, Pages 571-587, 2012.

[49] R. Pastor-Satorras, C. Castellano, P. Van Mieghem, and A. Vespignani, Epidemic processes in complex networks. Reviews of modern physics, 2015, vol. 87, no 3, p. 925.

[50] X. Pennec, P. Fillard, and N. Ayache, A Riemannian framework for tensor computing, International Journal of computer vision 66.1 (2006): 41-66.

[51] R.-E. Plessix, A review of the adjoint-state method for computing the gradient of a functional with geophysical applications, Geophys. J. Int. (2006) 167, 495-503.

[52] A. Portz and A. Seyfried, Analyzing Stop-and-Go Waves by Experiment and Modeling, Pedestrian and Evacuation Dynamics, pp. 577-586, Springer US, 2011.

[53] A. Proskurnikov, R. Tempo, A tutorial on modeling and analysis of dynamic social networks Part I. Annual Reviews in Control 43 (2017): 65-79.

[54] A. Proskurnikov, and R. Tempo, A tutorial on modeling and analysis of dynamic social networks Part II. Annual Reviews in Control 45 (2018): 166-190.

[55] A. Proskurnikov, A. Matveev, and M. Cao. Opinion dynamics in social networks with hostile camps: Consensus vs. polarization. IEEE Transactions on Automatic Control 61.6 (2015): 1524-1536.

[56] S. Ross, Introduction to probability models, Academic press, 2019.

[57] F. Santambrogio, Optimal Transport for Applied Mathematicians, Birkaüser, NY, 2015.

[58] V. Sekara, A. Stopczynski, S. Lehmann, Fundamental structures of dynamic social networks, Proceedings of the National Academy of Sciences Sep 2016, 113 (36).

[59] P. M. Soardi, Potential Theory on Infinite Networks, Springer-Verlag, 1994.

[60] J. Solomon, R. Rustamov, L. Guibas, and A. Butscher, Wasserstein propagation for semi-supervised learning. International Conference on Machine Learning, 2014. p. 306-314.

[61] G. Toscani, Kinetic models of opinion formation, Commun. Math. Sci., 4 (2006), 481-496.

[62] K. T. Sturm, Probability measures on metric spaces of nonpositive curvature. Heat Kernels and Analysis on Manifolds, Graphs, and Metric Spaces: Lecture Notes from a Quarter Program on Heat Kernels, Random Walks, and Analysis on Manifolds and Graphs: April 16-July 13, 2002, Emile Borel Centre of the Henri Poincaré Institute, Paris, France 338 (2003): 357.

[63] K. T. Sturm, A semigroup approach to harmonic maps, Potential Analysis 23.3 (2005): 225-277.

[64] L.-X. Wang, J. M. Mendel, Fuzzy Opinion Networks: A Mathematical Framework for the Evolution of Opinions and Their Uncertainties Across Social Networks, IEEE Transactions on Fuzzy Systems, Volume 24, Issue 4, Aug. 12016.

[65] D. J. Watts, and P. S. Dodds, Influentials, networks, and public opinion formation, Journal of consumer research, 2007, vol. 34 , no 4 , p. 441-458. 